February 1999

IMF Staff Country Report No. 99/8

\title{
Burundi: Statistical Annex
}

This Statistical Annex report on Burundi was prepared by a staff team of the International Monetary Fund as background documentation for the periodic consultation with this member country. As such, the views expressed in this document are those of the staff team and do not necessarily reflect the views of the Government of Burundi or the Executive Board of the IMF.

Copies of this report are available to the public from

International Monetary Fund • Publication Services

700 19th Street, N.W. - Washington, D.C. 20431

Telephone: (202) 623-7430 • Telefax: (202) 623-7201

Telex (RCA): 248331 IMF UR

E-mail: publications@imf.org

Internet: http://www.imf.org

Price: $\$ 15.00$ a copy

\section{International Monetary Fund Washington, D.C.}




\title{
INTERNATIONAL MONETARY FUND
}

\author{
BURUNDI \\ Statistical Annex \\ Prepared by a staff team consisting of Mr. Rothman (head), Mr. Bessaha, \\ Mr. Farah, and Ms. Nkusu (all AFR) \\ Approved by the African Department
}

December 21, 1998

Contents

Page

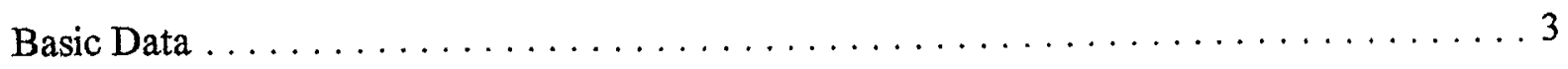

Social and Demographic Indicators $\ldots \ldots \ldots \ldots \ldots \ldots \ldots \ldots \ldots \ldots$

Tables

1. Gross Domestic Product at Current Prices, 1994-98 . . . . . . . . . . . . 6

2. Gross Domestic Product at Constant 1980 Prices, $1994-98$. . . . . . . . . . . 7

3. Supply and Use of Resources at Current Market Prices, 1994-98 . . . . . . . . 8

4. Savings and Investment, $1994-98 \ldots \ldots \ldots \ldots \ldots \ldots \ldots \ldots \ldots$

5. Key Coffee Sector Indicators, 1994/95-1998/99 . . . . . . . . . . . . . . 10

6. Illustrative Allocation of Arabica Coffee Receipts Among Producers

Intermediaries, and Stabilization Fund, 1994/95-1998/99 . . . . . . . . . . . 11

7. Arabica and Robusta Coffee Production, 1994/95-1998/99 . . . . . . . . . . 12

8. Cotton Production, Consumption, Exports, and Prices, 1994-98 . . . . . . . . 13

9. Tea Production, Exports, Stocks, and Prices, 1994-98 . . . . . . . . . . . . . 14

10. Production of the Manufacturing Industries, $1994-98 \ldots \ldots \ldots \ldots \ldots \ldots$

11. Energy Production, Imports, and Consumption, $1994-98 \ldots \ldots \ldots \ldots \ldots$

12. Retail Prices of Petroleum Products, $1994-98 \ldots \ldots \ldots \ldots \ldots \ldots \ldots$

13. Minimum Wages, $1994-98 \ldots \ldots \ldots \ldots \ldots \ldots \ldots \ldots \ldots \ldots \ldots \ldots$

14. Consumer Price Index for Households in Bujumbura, 1994-98 . . . . . . . . . . 19

15. Central Government Financial Operations, $1994-98 \ldots \ldots \ldots \ldots \ldots \ldots \ldots \ldots$

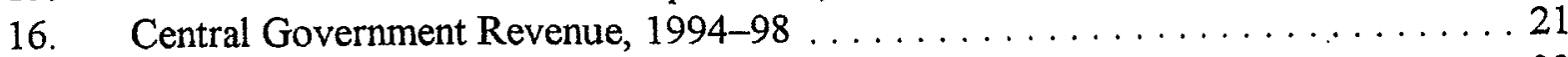

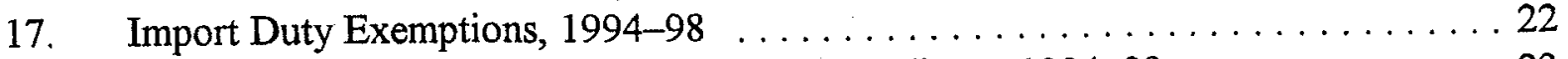

18. Economic Classification of Government Expenditure, 1994-98 . . . . . . . . 23

19. Expenditure on Health and Education, $1994-97 \ldots \ldots \ldots \ldots \ldots \ldots \ldots \ldots$

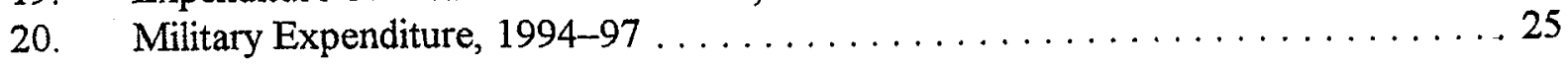


21. Functional Structure of Government Operating and Capital

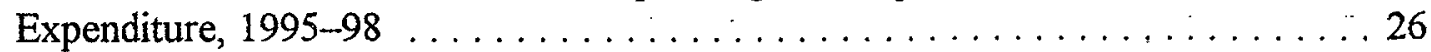

22. Structure of Central Government Employment and Salaries, 1996 . . . . . . . . 27

23. Size, Composition, and Gross Salaries of the Civil Service, $1983-96 \ldots \ldots \ldots .28$

24. Flows of On-Lending to, and Repayments by, Public Enterprises, 1994-98 . . . . 29

25. Outstanding Arrears on External Debt, $1995-98 \ldots \ldots \ldots \ldots \ldots \ldots \ldots \ldots$

26. Domestic Public Debt by Creditor and by Instrument, 1994-97 . . . . . . . . 31

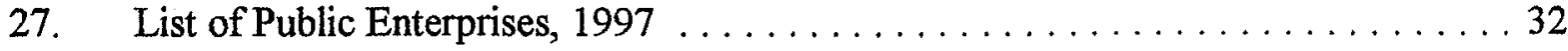

28. Main Economic Indicators of Public Enterprises, $1996 \ldots \ldots \ldots \ldots \ldots \ldots \ldots \ldots$

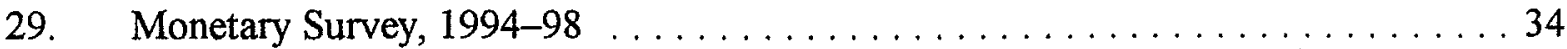

30. Summary Accounts of the Bank of the Republic of Burundi, 1994-98 . . . . . 35

31. Summary Accounts of Deposit Money Banks, 1994-98 . . . . . . . . . . . . 36

32. Summary Accounts of Burundi Development Bank (BNDE), Burundi Financing Company (SBF), Caisse Centrale de Mobilisation et de Financement

(CAMOFI), and Société de Gestion Financière (SOGEFI), 1994-98 . . . . . . 37

33. Outstanding Amount of Treasury Certificates, $1994-98 \ldots \ldots \ldots \ldots \ldots \ldots . \ldots 38$

34. Distribution of Credit to the Economy, $1994-98 \ldots \ldots \ldots \ldots \ldots \ldots . \ldots \ldots$

35. Structure of Interest Rates, $1994-98 \ldots \ldots \ldots \ldots \ldots \ldots$. . . . . . . . . . . . . 40

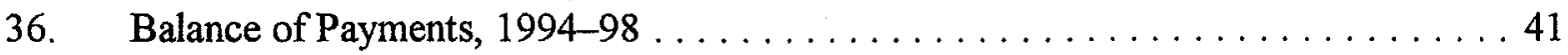

37. Composition of Exports, f.o.b., $1994-98 \ldots \ldots \ldots \ldots \ldots \ldots \ldots \ldots \ldots \ldots 42$

38. Composition of Imports, c.i.f., $1994-98 \ldots \ldots \ldots \ldots \ldots \ldots \ldots \ldots \ldots \ldots . \ldots . \ldots . \ldots 4$

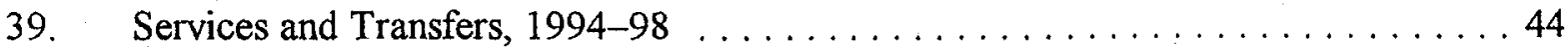

40. Structure, Volume, and Prices of International Trade, $1994-98 \ldots \ldots \ldots \ldots 45$

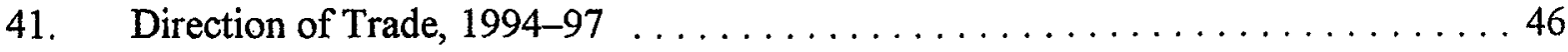

42. Trade Flows with the Preferential Trade Area Countries, 1994-97 . . . . . . . . 47

43. Outstanding Medium- and Long-Term Official External Debt, 1994-98 . . . . . . 48

44. Scheduled Debt Service, Principal, on Medium- and Long-Term Official

External Debt, $1994-98$. . . . . . . . . . . . . . . . . . . . . . . . . . . 49

45. Scheduled Debt Service, Interest, on Medium- and Long-Term Official External Debt, 1994-98 . . . . . . . . . . . . . . . . . . . . . . . . . 50

46. Outstanding Stock of External Debt and Average Terms on

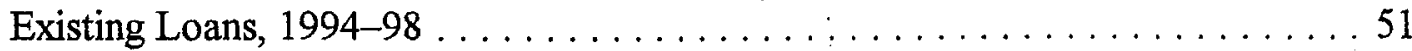

Figures

1. Selected Economic and Financial Indicators, $1994-99 \ldots \ldots \ldots \ldots$. . . . . . . 52

2. Selected Exchange Rate Indices, January 1990-August 1998 . . . . . . . . . . . 53

Appendixes

I. Summary of Exchange and Trade System $\ldots \ldots \ldots \ldots \ldots \ldots \ldots \ldots \ldots$

II. Summary of the Tax System, end-June $1998 \ldots \ldots \ldots \ldots \ldots \ldots \ldots \ldots \ldots \ldots$ 
Burundi: Basic Data

\begin{tabular}{|c|c|c|c|c|c|}
\hline & 1994 & 1995 & 1996 & $\begin{array}{r}1997 \\
\text { Est. }\end{array}$ & $\begin{array}{l}1998 \\
\text { Proj. }\end{array}$ \\
\hline & \multicolumn{5}{|c|}{ (In billions of Burundi francs) } \\
\hline \multicolumn{6}{|l|}{$\begin{array}{l}\text { National accounts } \\
\text { Gross domestic product in nominal terms at }\end{array}$} \\
\hline factor cost & 203.1 & 218.1 & 248.2 & 306.4 & 386.9 \\
\hline Primary sector & 95.2 & 105.0 & 136.0 & 163.4 & 191.1 \\
\hline Secondary sector & 45.7 & 42.0 & 38.1 & 51.2 & 73.8 \\
\hline Tertiary sector & 62.2 & 71.1 & $\mathbf{7 4 . 0}$ & 91.8 & 122.1 \\
\hline Gross domestic product at current market prices & 233.3 & 249.9 & 272.6 & 337.3 & 425.1 \\
\hline Imports of goods and nonfactor services & 66.6 & 68.2 & 41.5 & 47.9 & 62.0 \\
\hline Total supply of resources $=$ total use & 299.9 & 318.0 & 314.0 & 385.2 & 487.1 \\
\hline Exports of goods and nonfactor services & 24.0 & 32.2 & 15.2 & 33.7 & 24.8 \\
\hline Domestic demand & 275.9 & 285.8 & 298.9 & 351.5 & 462.3 \\
\hline Private consumption & 218.7 & 228.3 & 231.5 & 275.3 & 381.5 \\
\hline Public consumption & 32.5 & 33.6 & 34.5 & 49.0 & 47.0 \\
\hline Gross investment & 24.7 & 23.9 & 32.8 & 27.2 & 33.9 \\
\hline Of which: gross fixed capital formation & 24.7 & 24.1 & 33.2 & 24.5 & 30.4 \\
\hline \multirow[t]{2}{*}{ Resource gap } & -42.6 & -36.0 & -26.3 & -14.2 & -37.2 \\
\hline & \multicolumn{5}{|c|}{ (Annual percentage change) } \\
\hline Gross domestic product and prices & & & & & \\
\hline Real GDP (market prices) & -3.7 & -7.3 & -8.4 & 0.4 & 4.5 \\
\hline GDP deflator & 5.6 & 15.2 & 19.4 & 23.3 & 20.6 \\
\hline \multirow[t]{2}{*}{ Consumer price index } & 14.9 & 19.4 & 26.4 & 31.1 & 17.0 \\
\hline & \multicolumn{5}{|c|}{ (In billions of Burundi francs) } \\
\hline Central government operations & & & & & \\
\hline Revenue and grants & 47.9 & 53.2 & 48.5 & 56.6 & 64.5 \\
\hline Revenue & 42.2 & 44.1 & 42.3 & 46.3 & 53.2 \\
\hline Grants & 5.7 & 9.1 & 6.1 & 10.3 & 11.3 \\
\hline Expenditure and net lending & 57.9 & 65.3 & 76.0 & 74.9 & 82.5 \\
\hline Current expenditure & 39.1 & 45.1 & 48.1 & 61.4 & 61.2 \\
\hline Capital expenditure and net lending & 18.8 & 20.2 & 27.9 & 13.5 & 21.3 \\
\hline \multicolumn{6}{|l|}{ Overall balance (cash basis) } \\
\hline Including grants & -9.7 & -7.6 & -17.5 & -12.3 & -7.1 \\
\hline Excluding grants & -15.4 & -16.7 & -23.6 & -22.6 & -18.4 \\
\hline Financing & 9.7 & 7.6 & 17.5 & 12.3 & 7.1 \\
\hline Foreign (net) & 6.3 & 3.6 & 2.2 & 1.3 & 3.5 \\
\hline Domestic (net) & 3.4 & 4.0 & 15.3 & 11.0 & 3.6 \\
\hline Banking system & 3.7 & -2.9 & 11.1 & 11.0 & 3.6 \\
\hline \multirow[t]{2}{*}{ Other } & -0.3 & 6.9 & 4.2 & 0.0 & 0.0 \\
\hline & \multicolumn{5}{|c|}{ (In percent of GDP) } \\
\hline Revenue (excluding grants) & 18.1 & 17.7 & 15.5 & 13.7 & 12.5 \\
\hline Grants & 2.4 & 3.6 & 2.3 & 3.1 & 2.7 \\
\hline Current expenditure & 16.8 & 18.0 & 17.6 & 18.2 & 14.4 \\
\hline Capital expenditure & 9.0 & 8.3 & 10.4 & 5.0 & 5.0 \\
\hline Total expenditure and net lending & 24.8 & 26.1 & 27.9 & 22.2 & 19.4 \\
\hline \multicolumn{6}{|l|}{ Overall balance (commitment basis) } \\
\hline Including grants & -4.3 & -4.9 & -10.1 & -5.4 & -4.2 \\
\hline Excluding grants & .6 .7 & -8.5 & -12.4 & -8.5 & -6.9 \\
\hline
\end{tabular}


Burundi: Basic Data (concluded)

\begin{tabular}{|c|c|c|c|c|c|}
\hline & 1994 & 1995 & 1996 & $\begin{array}{r}1997 \\
\text { Est. }\end{array}$ & $\begin{array}{l}1998 \\
\text { Proj. }\end{array}$ \\
\hline & \multicolumn{5}{|c|}{ (In billions of Burundi francs) } \\
\hline \multicolumn{6}{|l|}{ Money and credit } \\
\hline Foreign assets (net) & 37.3 & 45.2 & 37.1 & 35.6 & 36.3 \\
\hline Domestic credit & 42.1 & 35.5 & 54.7 & 68.0 & 84.8 \\
\hline Government (net) & 1.0 & 1.0 & 11.7 & 23.4 & 27.2 \\
\hline Credit to the private sector & 38.4 & 33.2 & 41.3 & 42.4 & 55.5 \\
\hline Credit to public enterprises & 2.7 & 1.3 & 1.7 & 2.2 & 2.2 \\
\hline Money and quasi money & 52.3 & 50.0 & 57.2 & 63.1 & 70.9 \\
\hline \multirow[t]{2}{*}{ Other items (net) } & -27.0 & -30.7 & -34.6 & -40.5 & -50.3 \\
\hline & \multicolumn{5}{|c|}{$\begin{array}{l}\text { (Annual change in percent of the beginning-of-period } \\
\text { broad money supply) }\end{array}$} \\
\hline Net foreign assets & 28.9 & 15.1 & -16.2 & -2.6 & 1.2 \\
\hline Domestic credit & 17.3 & -12.5 & 38.3 & 23.4 & 26.6 \\
\hline Net credit to the government & 9.0 & 0.1 & 21.4 & 20.4 & 6.0 \\
\hline Credit to the private sector & 5.3 & -9.9 & 16.2 & 2.0 & 20.6 \\
\hline Credit to public enterprises & 3.0 & -2.7 & 0.7 & 0.9 & -0.1 \\
\hline \multirow[t]{2}{*}{ Money and quasi money } & 35.0 & -4.5 & 14.4 & 10.4 & 12.2 \\
\hline & \multicolumn{5}{|c|}{ (In millions of U.S. dollars) } \\
\hline $\begin{array}{l}\text { Balance of payments } \\
\text { Trade balance }\end{array}$ & -91.9 & -63.0 & -59.9 & -10.6 & -53.2 \\
\hline Exports, f.o.b. & 80.7 & 112.5 & 40.1 & 87.3 & 49.3 \\
\hline Of which: coffee & 57.2 & 89.1 & 28.8 & 76.6 & 38.6 \\
\hline Imports, f.o.b. & -172.6 & -175.6 & -100.0 & -97.9 & -102.6 \\
\hline Services (net) & -87.9 & -93.6 & -40.8 & -40.4 & -42.0 \\
\hline Private transfers (net) & 17.4 & 15.6 & 11.2 & 8.7 & 11.0 \\
\hline Current account (excluding official transfers) & -162.4 & -141.0 & -89.4 & -42.3 & -84.2 \\
\hline Official transfers & 143.8 & 136.1 & 49.8 & 50.9 & 50.9 \\
\hline Current account (including official transfers) & -18.6 & -4.9 & -39.6 & 8.6 & -33.3 \\
\hline Capital account & 62.4 & 7.3 & -43.4 & -39.8 & 8.0 \\
\hline Medium- and long-term official loans (net) & 25.2 & 4.9 & 7.3 & 3.5 & 8.0 \\
\hline Direct investment & -0.1 & 1.4 & 0 & 0 & 0 \\
\hline Other capital l/ & 37.3 & 1.0 & -50.7 & -43.3 & 0.0 \\
\hline \multirow[t]{2}{*}{ Overall balance } & 43.8 & 2.4 & -83.0 & -31.3 & -25.3 \\
\hline & \multicolumn{5}{|c|}{ (As a percent of GDP, unless otherwise indicated) } \\
\hline Gross official reserves & & & & & \\
\hline $\begin{array}{l}\text { In millions of U.S. dollars } \\
\text { In months of imports of goods and }\end{array}$ & 213.9 & 215.3 & 144.7 & 117.3 & 108.2 \\
\hline nonfactor services & 8.2 & 7.8 & 10.8 & 9.6 & 8.6 \\
\hline Exports, f.o.b. & 8.7 & 11.2 & 4.5 & 6.5 & 5.0 \\
\hline Imports, c.i.f. & -18.7 & -17.6 & -11.1 & -7.3 & -10.5 \\
\hline Current account (excluding official transfers) & -17.6 & -14.1 & -9.9 & -4.4 & -8.6 \\
\hline \multirow[t]{2}{*}{ Overall balance of payments } & 4.7 & 0.2 & -9.2 & -3.3 & -2.6 \\
\hline & \multicolumn{5}{|c|}{ (In millions of U.S. dollars, unless otherwise indicated) } \\
\hline Total external debt $2 /$ & $1,108.7$ & $1,183.4$ & $1,175.6$ & $1,184.1$ & $1,200.3$ \\
\hline Scheduled debt service 3 / & 39.6 & 39.3 & 105.9 & 36.3 & 57.9 \\
\hline Exchange rate (FBu/US\$; period average) & 252.7 & 249.8 & 302.8 & 352.4 & 435.2 \\
\hline
\end{tabular}

Sources: Burundi authorities; and Fund staff estimates and projections.

1/ Including net short-term capital and errors and omissions.

2/ Outstanding public and publicly guaranteed debt, including IMF.

3 / In percent of exports of goods and nonfactor services. 
Burundi: Social and Demographic Indicators 1/

\begin{tabular}{lr}
\hline Land area (sq. km.) & $25,680.0$ \\
Population & \\
Total (in millions; 1997) & 6.2 \\
$\quad$ Urban population (percent of total) & 7.8 \\
Rural population (percent of total) & 92.2 \\
Density (people per sq. km.) & 241 \\
Growth rate (percent) & 1.8 \\
GDP per capita (US\$; 1997) & 154 \\
Life expectancy at birth (years) & \\
Overall & 50.3 \\
Men & 48.6 \\
Women & 52.1 \\
Crude birth rate (per 1,000) & 42.5 \\
Crude death rate (per 1,000) & 17.0 \\
Infant mortality rate (per 1,000) & 97.2 \\
Education (1996) & \\
Gross primary enrollment rate (percent) & 43.2 \\
Gross secondary enrollment rate (percent) & 6.0 \\
Adult literacy rate (percent) & 35.0 \\
School population & \\
Primary & 453,746 \\
Secondary (general) & 56,887 \\
Secondary (technical and teacher education) & 5,712 \\
Higher education & 4,379 \\
Health (1996) & \\
Physicians (in hospitals) & 329 \\
Hospitals & 34 \\
Population per physician & 17,210 \\
Population per nurse & 4,800 \\
Population per hospital/bed & 1,526 \\
Access to safe water (percent) & 37.8 \\
Immunization rate (percent) & 63.0 \\
Urban areas & 92.0 \\
Rural areas & 33.9 \\
\hline & \\
\hline
\end{tabular}

Sources: Burundi authorities; World Bank, World Development Indicators; and IMF, International Financial Statistics.

1/ Most recent estimates available. 
Table 1. Burundi: Gross Domestic Product at Current Prices, 1994-98

(In millions of Burundi francs, unless otherwise indicated)

\begin{tabular}{|c|c|c|c|c|c|}
\hline & 1994 & 1995 & 1996 & 1997 & 1998 \\
\hline & \multicolumn{4}{|c|}{ Estimates } & Proj. \\
\hline Primary sector & 95,188 & 105,035 & 136,018 & 163,354 & 191,069 \\
\hline Food crops & 67,975 & 81,473 & 107,448 & 130,586 & 155,855 \\
\hline Livestock & 9,832 & 8,553 & 10,223 & 12,254 & 14,688 \\
\hline Fishing & 533 & 571 & 598 & 552 & 1,060 \\
\hline Forestry & 3,841 & 3,341 & 3,425 & 3,741 & 3,000 \\
\hline Export crops & 13,007 & 11,097 & 14,324 & 16,222 & 16,467 \\
\hline Secondary sector & 45,746 & 41,979 & 38,139 & 51,217 & 73,787 \\
\hline Manufacturing & 21,857 & 20,635 & 16,500 & 20,290 & 28,536 \\
\hline Agricultural processing & 1,976 & 1,104 & 1,702 & 2,175 & 2,762 \\
\hline Food industries & 11,957 & 11,304 & 9,021 & 11,526 & 12,056 \\
\hline Textiles and leather products & 2,062 & 2,318 & 1,753 & 3,518 & 4,496 \\
\hline Other industry & 5,862 & 5,909 & 4,024 & 3,071 & 9,221 \\
\hline Handicrafts & 7,927 & 5,709 & 6,182 & 9,302 & 15,241 \\
\hline Construction & 14,588 & 14,339 & 13,970 & 19,193 & 26,523 \\
\hline Mining and energy & 1,374 & 1,296 & 1,487 & 2,432 & 3,487 \\
\hline Tertiary sector & 62,186 & 71,123 & 74,025 & 91,861 & 122,065 \\
\hline Public services & 39,832 & 41,728 & 43,706 & 57,757 & 76,164 \\
\hline Transport and communications & 8,014 & 10,440 & 12,598 & $13 ; 593$ & 14,479 \\
\hline Commerce & 9,339 & 12,957 & 10,884 & 13,165 & 18,005 \\
\hline Other services & 5,001 & 5,998 & 6,837 & 7,347 & 13,416 \\
\hline GDP at factor cost & 203,120 & 218,137 & 248,182 & 306,432 & 386,920 \\
\hline Indirect taxes, net of subsidies & 30,200 & 31,728 & 24,400 & 30,900 & 38,200 \\
\hline GDP at market prices & 233,320 & 249,865 & 272,582 & 337,332 & 425,120 \\
\hline Growth rate (in percent) & 14.1 & 7.1 & 9.1 & 23.8 & 26.0 \\
\hline \multicolumn{6}{|l|}{ Memorandum items: } \\
\hline At factor cost & 188.3 & 217.2 & 258.6 & 318.3 & 387.6 \\
\hline At market prices & 190.6 & 219.6 & 261.4 & 322.3 & 388.7 \\
\hline Annual change (in percent) & 5.6 & 15.2 & 19.4 & 23.3 & 20.6 \\
\hline
\end{tabular}

Sources: Burundi authorities; and Fund staff estimates and projections. 
Table 2. Burundi: Gross Domestic Product at Constant 1980 Prices, 1994-98

(In millions of Burundi francs, unless otherwise indicated)

\begin{tabular}{|c|c|c|c|c|c|}
\hline & 1994 & 1995 & 1996 & 1997 & \multirow{2}{*}{$\begin{array}{l}1998 \\
\text { Proj. }\end{array}$} \\
\hline & \multicolumn{4}{|c|}{ Estimates } & \\
\hline Primary sector & 59,508 & 55,916 & 56,734 & 57,048 & 59,856 \\
\hline Food crops & 44,797 & 44,484 & 45,996 & 46,456 & 49,801 \\
\hline Livestock & 4,397 & 3,718 & 3,347 & 3,380 & 3,448 \\
\hline Fishing & 96 & 91 & 64 & 51 & 52 \\
\hline Forestry & 3,495 & 3,320 & 3,154 & 2,996 & 3,056 \\
\hline Export crops & 6,722 & 4,302 & 4,173 & 4,165 & 3,499 \\
\hline Secondary sector & 13,841 & 11,468 & 9,953 & 9,868 & 10,050 \\
\hline Manufacturing & 4,838 & 4,168 & 3,216 & 2,949 & 3,008 \\
\hline Agricultural processing & 718 & 459 & 445 & 468 & 477 \\
\hline Food industries & 703 & 633 & 474 & 427 & 436 \\
\hline Textiles and leather products & 635 & 572 & 543 & 546 & 557 \\
\hline Other industry & 2,783 & 2,504 & 1,753 & 1,508 & 1,538 \\
\hline Handicrafts & 3,534 & 2,378 & 2,259 & 2,372 & 2,420 \\
\hline Construction & 4,558 & 4,193 & 3,858 & 3,896 & 3,943 \\
\hline Mining and energy & 911 & 729 & 619 & 650 & 679 \\
\hline Tertiary sector & 34,548 & 33,042 & 29,275 & 29,352 & 29,925 \\
\hline Public services & 21,433 & 20,790 & 20,582 & 20,606 & 21,018 \\
\hline Transportation and communications & 2,843 & 2,758 & 2,465 & 2,449 & 2,498 \\
\hline Commerce & $\overrightarrow{8,671}$ & 7,977 & 4,786 & 4,834 & 4,931 \\
\hline Other services & 1,601 & 1,517 & 1,442 & 1,463 & 1,478 \\
\hline GDP at factor cost & 107,896 & 100,426 & 95,961 & 96,268 & 99,830 \\
\hline Indirect taxes, net of subsidies & 14,809 & 13,364 & 8,315 & 8,398 & 9,553 \\
\hline GDP at market prices & 122,705 & 113,790 & 104,276 & 104,666 & 109,384 \\
\hline Growth rate (in percent) & -3.7 & -7.3 & -8.4 & 0.4 & 4.5 \\
\hline
\end{tabular}

Sources: Burundi authorities; and Fund staff estimates and projections. 
-8 -

Table 3. Burundi: Supply and Use of Resources at Current Market Prices, 1994-98

\begin{tabular}{|c|c|c|c|c|c|}
\hline & 1994 & 1995 & 1996 & 1997 & \multirow{2}{*}{$\begin{array}{l}1998 \\
\text { Proj. }\end{array}$} \\
\hline & \multicolumn{4}{|c|}{ Estimates } & \\
\hline & \multicolumn{5}{|c|}{ (In millions of Burundi francs) } \\
\hline GDP at market prices & 233,320 & 249,865 & 272,582 & 337,332 & 425,120 \\
\hline Resource gap & $-42,560$ & $-35,976$ & $-26,271$ & $-14,202$ & $-37,210$ \\
\hline Imports of goods and nonfactor services & 66,593 & 68,173 & 41,453 & 47,891 & 61,972 \\
\hline Exports of goods and nonfactor services & 24,034 & 32,197 & 15,182 & 33,689 & 24,763 \\
\hline Gross domestic expenditure & 275,880 & 285,840 & 298,853 & 351,534 & 462,329 \\
\hline Consumption & 251,147 & 261,920 & 266,021 & 324,325 & 428,456 \\
\hline Public & 32,459 & 33,578 & 34,474 & 49,000 & 47,000 \\
\hline Private & 218,688 & 228,342 & 231,547 & 275,325 & 381,456 \\
\hline Gross investment & 24,733 & 23,920 & 32,832 & 27,209 & 33,873 \\
\hline Fixed investment & 24,680 & 24,119 & 33,241 & 24,470 & 30,429 \\
\hline Public & 21,000 & 20,700 & 28,400 & 16,900 & 21,300 \\
\hline Private & 3,680 & 3,419 & 4,841 & 7,570 & 9,129 \\
\hline Changes in stocks & 53 & -199 & -409 & 2,739 & 3,443 \\
\hline Gross domestic savings & $-17,827$ & $-12,055$ & 6,562 & 13,007 & $-3,337$ \\
\hline Government savings & 3,100 & $-1,000$ & $-5,800$ & $-15,100$ & $-8,000$ \\
\hline \multirow[t]{2}{*}{ Private savings } & $-20,927$ & $-11,055$ & 12,362 & 28,107 & 4,663 \\
\hline & \multicolumn{5}{|c|}{ (In percent of GDP) } \\
\hline Resource gap & -18.2 & -14.4 & -9.6 & -4.2 & -8.8 \\
\hline Imports of goods and nonfactor services & 28.5 & 27.3 & 15.2 & 14.2 & 14.6 \\
\hline Exports of goods and nonfactor services & 10.3 & 12.9 & 5.6 & 10.0 & 5.8 \\
\hline Gross domestic expenditure & 118.2 & 114.4 & 109.6 & 104.2 & 108.8 \\
\hline Consumption & 107.6 & 104.8 & 97.6 & 96.1 & 100.8 \\
\hline Public & 13.9 & 13.4 & 12.6 & 14.5 & 11.1 \\
\hline Private & 93.7 & 91.4 & 84.9 & 81.6 & 89.7 \\
\hline Gross investment & 10.6 & 9.6 & 12.0 & 8.1 & 8.0 \\
\hline Fixed investment & 10.6 & 9.7 & 12.2 & 7.3 & 7.2 \\
\hline Public & 9.0 & 8.3 & 10.4 & 5.0 & 5.0 \\
\hline Private & 1.6 & 1.4 & 1.8 & 2.2 & 2.1 \\
\hline Changes in stocks & 0.0 & -0.1 & -0.1 & 0.8 & 0.8 \\
\hline Gross domestic savings & -7.6 & -4.8 & 2.4 & 3.9 & -0.8 \\
\hline Government savings & 1.3 & -0.4 & -2.1 & -4.5 & -1.9 \\
\hline Private savings & -9.0 & -4.4 & 4.5 & 8.3 & 1.1 \\
\hline
\end{tabular}

Sources: Burundi authorities; and Fund staff estimates and projections. 
Table 4. Burundi: Savings and Investment, 1994-98

\begin{tabular}{|c|c|c|c|c|c|}
\hline & 1994 & 1995 & 1996 & 1997 & 1998 \\
\hline & \multicolumn{4}{|c|}{ Estimates } & Proj. \\
\hline & \multicolumn{5}{|c|}{ (In millions of Burundi francs at current prices) } \\
\hline Net factor services & 2,881 & 3,147 & 4,239 & 3,771 & 4,265 \\
\hline Private current transfers & 4,397 & 3,897 & 3,391 & 3,066 & 4,787 \\
\hline External current account 1 / & 41,038 & 35,222 & 27,070 & 14,907 & 36,644 \\
\hline Official transfers & 36,338 & 33,998 & 15,079 & 17,937 & 22,152 \\
\hline External current account $2 /$ & 4,700 & 1,224 & 11,991 & 3,031 & 14,492 \\
\hline Gross domestic savings & $-17,827$ & $-12,055$ & 6,562 & 13,007 & $-3,337$ \\
\hline Gross national savings & 25,789 & 28,987 & 29,272 & 37,781 & 27,867 \\
\hline \multirow[t]{2}{*}{ Gross investment (including stocks) } & 24,733 & 23,920 & 32,832 & 27,209 & 33,873 \\
\hline & \multicolumn{5}{|c|}{ (In percent) } \\
\hline Gross domestic savings/gross investment & -72.1 & -50.4 & 20.0 & 47.8 & -9.9 \\
\hline Gross national savings/gross investment & 104.3 & 121.2 & 89.2 & 138.9 & 82.3 \\
\hline Gross investment/GDP & 10.6 & 9.6 & 12.0 & 8.1 & 8.0 \\
\hline Gross national savings/GDP & 11.1 & 11.6 & 10.7 & 11.2 & 6.6 \\
\hline External current account/GDP $1 /$ & 17.6 & 14.1 & 9.9 & 4.4 & 8.6 \\
\hline External current account/GDP $2 /$ & 2.0 & 0.5 & 4.4 & 0.9 & 3.4 \\
\hline \multicolumn{6}{|l|}{ Memorandum item: } \\
\hline Nominal GDP (in millions of Burundi francs) & 233,320 & 249,865 & 272,582 & 337,332 & 425,120 \\
\hline
\end{tabular}

Sources: Burundi authorities; and Fund staff estimates and projections.

1/ Excluding official transfers.

2/ Including official transfers. 
Table 5. Burundi: Key Coffee Sector Indicators, 1994/95-1998/99 1/

\begin{tabular}{|c|c|c|c|c|c|}
\hline & $1994 / 95$ & $1995 / 96$ & $1996 / 97$ & Est. & $\begin{array}{r}1998 / 99 \\
\text { Proj. }\end{array}$ \\
\hline Production (green coffee; metric tons) & 41,293 & 25,565 & 26,876 & 20,196 & 17,436 \\
\hline Fully washed & 26,708 & 16,382 & 18,103 & 12,007 & 10,601 \\
\hline Semiwashed & 14,278 & 8,815 & 8,631 & 7,984 & 6,636 \\
\hline Robusta & 307 & 369 & 142 & 204 & 200 \\
\hline Exports of coffee (millions of U. S. dollars) & 57.2 & 89.1 & 28.8 & 76.6 & 38.6 \\
\hline Exports of coffee/total exports (in percent) & 70.9 & 79.2 & 71.8 & 87.7 & 78.7 \\
\hline Taxes on coffee/total revenue (in percent) & 13.6 & 11.3 & 3.4 & 1.4 & 10.3 \\
\hline Taxes on coffee/GDP (in percent) & 2.3 & 1.9 & 0.4 & 0.2 & 1.2 \\
\hline \multicolumn{6}{|l|}{ Producer price (Burundi francs per kilogram) } \\
\hline Fully washed (Burundi francs per kilogram) & 280.5 & 280.5 & 290.0 & 350.0 & 450.0 \\
\hline Semiwashed (Burundi francs per kilogram) & 180.0 & 180.0 & 290.0 & 330.0 & 420.0 \\
\hline \multicolumn{6}{|l|}{ Overall surplus or deficit of the coffee sector } \\
\hline (in millions of Burundi francs) & $6,636.3$ & 526.6 & -711.2 & 49.5 & $-1,691.8$ \\
\hline \multicolumn{6}{|l|}{ Memorandum items: } \\
\hline \multicolumn{6}{|l|}{ World market prices $2 /$} \\
\hline Cents/lb. & 149 & 149 & 120 & 185 & 135 \\
\hline Index $(1990=100)$ & 168 & 168 & 135 & 209 & 152 \\
\hline \multicolumn{6}{|l|}{ Producer price index $(1990 / 91=100) 3 /$} \\
\hline Nominal & 117 & 117 & 121 & 146 & 188 \\
\hline Real 4/ & 91 & 76 & 63 & 58 & 63 \\
\hline
\end{tabular}

Sources: Burundi authorities; and Fund staff estimates and projections.

1/ The coffee crop year extends from May 1 to April 30.

2/ First year of Burundi season; e.g., for the 1994/95 season, the 1994 price is reported. Prices are for mild coffee, i.e., including arabica.

3/ For fully washed coffee.

4/ Deflated by the consumer price index (January 1991=100). Price indices are for the first year of the season; e.g., for the season 1994/95, the 1994 deflator is used. 
Table 6. Burundi: Illustrative Allocation of Arabica Coffee Receipts Among Producers, Intermediaries, and Stabilization Fund, 1994/95-1998/99 1/

\begin{tabular}{|c|c|c|c|c|c|}
\hline & $1994 / 95$ & $1995 / 96$ & $\underline{1996 / 97}$ & $\frac{1997 / 98}{\mathrm{t} .}$ & $\begin{array}{r}1998 / 99 \\
\text { Proj. }\end{array}$ \\
\hline 1. Producer prices & \multicolumn{5}{|c|}{$\begin{array}{c}\text { (In Burundi francs per kilogram of coffee, } \\
\text { unless otherwise indicated) }\end{array}$} \\
\hline \multicolumn{6}{|l|}{ Parchment coffee price } \\
\hline Semiwashed coffee price & 180.0 & 180.0 & 290.0 & $\mathbf{3 3 0 . 0}$ & 420.0 \\
\hline Fully washed coffee price & 280.5 & 280.5 & 290.0 & 350.0 & 450.0 \\
\hline Green coffee price & 305.0 & 309.6 & 366.8 & 431.7 & 553.1 \\
\hline 2. Transportation charges & 6.1 & 12.3 & 17.3 & 22.4 & 25.0 \\
\hline 3. Production and processing charges & 98.5 & 95.1 & 152.0 & 180.1 & 201.8 \\
\hline Intermediaries & 59.7 & 62.7 & 105.3 & 127.4 & 149.6 \\
\hline Wholesalers & 38.8 & 32.4 & 46.7 & 52.7 & 52.2 \\
\hline 4. OCIBU levies & 34.7 & 70.2 & 74.2 & 134.2 & 145.2 \\
\hline 5. Green coffee cost price ex factory $(1+2+3+4)$ & 444.3 & 487.2 & 610.3 & 768.5 & 925.1 \\
\hline 6. Export costs and charges & 236.7 & 86.8 & 87.5 & 237.2 & 145.6 \\
\hline Transport and handling & 20.3 & $\ldots$ & $\ldots$ & $\ldots$ & $\ldots$ \\
\hline Commissions & $\ldots$ & $\ldots$ & $\ldots$ & $\ldots$ & $\ldots$ \\
\hline Other export costs & $\ldots$ & $\ldots$ & $\ldots$ & $\ldots$ & ... \\
\hline Export taxes & 216.4 & 86.8 & 87.5 & 237.2 & 145.6 \\
\hline 7. Green coffee cost price at port of exports $(5+6)$ & 681.0 & 574.1 & 697.7 & $1,005.7$ & $1,070.7$ \\
\hline 8. Average green coffee selling price $2 /$ & 843.2 & 595.3 & 670.5 & $1,008.2$ & 972.5 \\
\hline 9. Profit or loss (-) (8-7) & 162.2 & 21.3 & -27.2 & 2.5 & -98.2 \\
\hline 10. Total exportable green coffee (in metric tons) & $40,914.0$ & $24,755.9$ & $26,118.8$ & $19,652.9$ & $17,236.4$ \\
\hline $\begin{array}{l}\text { 11. Global profit or loss }(-)(9 \times 10) \\
\text { (in millions of Burundi francs) }\end{array}$ & $6,636.3$ & 526.6 & -711.2 & 49.5 & $-1,691.8$ \\
\hline Producer prices for arabica parchment coffee & \multicolumn{5}{|c|}{ (U.S. dollars per kilogram) } \\
\hline Burundi & 0.71 & 0.72 & 0.96 & 0.93 & 0.97 \\
\hline Rwanda & 1.36 & 1.14 & $\ldots$ & 1.16 & $\ldots$ \\
\hline Tanzania & 1.83 & 1.72 & 1.06 & & $\ldots$ \\
\hline Uganda & 1.68 & 0.98 & 1.15 & 1.20 & ... \\
\hline Memorandum items: & \multicolumn{5}{|c|}{ (Units as indicated) } \\
\hline World market price (cents/lb.) & 149 & 149 & 120 & 185 & 135 \\
\hline \multicolumn{6}{|l|}{ Ratio of producer price to export price (percent) } \\
\hline Semiwashed coffee price & 21.35 & 30.23 & 43.25 & 32.73 & 43.19 \\
\hline Fully washed coffee price & 33.27 & 47.12 & 43.25 & 34.71 & 46.27 \\
\hline Green coffee price & 36.17 & 52.00 & 54.71 & 42.82 & 56.87 \\
\hline \multicolumn{6}{|l|}{ Real producer prices $3 /$} \\
\hline Washed coffee price & 140.41 & 117.65 & 149.95 & 130.13 & 141.60 \\
\hline Fully washed coffee price & 218.80 & 183.33 & 149.95 & 138.01 & 151.72 \\
\hline Green coffee price & 237.91 & 202.35 & 189.66 & 170.23 & 186.48 \\
\hline Coffee exports volume index $(1990 / 91=100)$ & 132.25 & 80.02 & 84.43 & 63.53 & 55.72 \\
\hline
\end{tabular}

Source: Office des Cultures Industrielles du Burundi (OCIBU).

1/ The coffee crop year extends from May 1 to April 30.

2/ Determined as an average rate, on the basis of actual payments in Dar es Salaam in relation to exportable coffee output.

3/ Deflated by the consumer price index (January 1991=100) 
Table 7. Burundi: Arabica and Robusta Coffee Production, 1994/95-1998/99 1/ (In metric tons)

\begin{tabular}{|c|c|c|c|c|c|}
\hline & $1994 / 95$ & $1995 / 96$ & \multicolumn{2}{|c|}{ Est. } & $\begin{array}{r}1998 / 99 \\
\text { Proj. }\end{array}$ \\
\hline Arabica fully washed & 26,708 & 16,381 & 18,103 & 12,007 & 10,600 \\
\hline F.W. Super 2/ & 756 & 2,322 & 666 & 1,008 & 890 \\
\hline F.W. Extra $2 /$ & 5,586 & 3,726 & 2,249 & 2,556 & 2,257 \\
\hline F.W.H.T./Courant 1 & 8,089 & 4,983 & 12,024 & 5,736 & 5,064 \\
\hline F.W. /Courant 2 & 10,036 & 3,305 & $\ldots$ & $\ldots$ & $\ldots$ \\
\hline F.W.4./Brisure & 1,364 & 360 & 444 & 174 & 154 \\
\hline F.W.5./Triage and Brisures & 877 & & 227 & 144 & 127 \\
\hline F.W.Stock/H. Normes & $\cdots$ & 1,685 & 2,492 & 2,389 & 2,109 \\
\hline Arabica semiwashed & 14,278 & 8,815 & 8,631 & 7,984 & 6,636 \\
\hline W.023/ & 774 & 1,080 & 225 & 90 & 75 \\
\hline W.3A 3/ & 2,124 & 1,188 & 681 & 311 & 258 \\
\hline W.3B & 5,405 & 3,365 & 2,645 & 1,998 & 1,661 \\
\hline W.HTM/3C & 5,121 & 1,782 & 3,041 & 2,009 & 1,669 \\
\hline W.4/Brisure & 594 & 198 & 405 & 300 & 249 \\
\hline W.5/Triage and Brisures & 260 & $\ldots$ & 189 & 130 & 108 \\
\hline W.H./Normes & $\ldots$ & 1,153 & 1,446 & 3,146 & 2,615 \\
\hline Total arabica & 40,986 & 25,147 & 26,733 & 19,991 & 17,236 \\
\hline Robusta & 307 & 369 & 142 & 204 & 200 \\
\hline Total coffee production & 41,293 & 25,566 & 26,876 & 20,196 & 17,436 \\
\hline
\end{tabular}

Source: Office des Cultures Industrielles du Burundi (OCIBU).

1/ The coffee crop year extends from May 1 to April 30.

2/ High-quality, fully washed arabica.

3/ High-quality washed arabica. 
Table 8. Burundi: Cotton Production, Consumption, Exports, and Prices, 1994-98 1/ (In units indicated)

\begin{tabular}{|c|c|c|c|c|c|}
\hline & 1994 & 1995 & 1996 & $\begin{array}{r}1997 \\
\text { Est. }\end{array}$ & $\begin{array}{c}1998 \\
\text { Proj. }\end{array}$ \\
\hline \multicolumn{6}{|l|}{ Production } \\
\hline Area under cultivation (hectares) & 6,067 & 6,168 & 4,070 & 3,457 & 3,596 \\
\hline North & 1,950 & 905 & 1,140 & 515 & 784 \\
\hline Center & 1,051 & 760 & 338 & 130 & 222 \\
\hline South & 2,193 & 2,228 & 740 & 1,082 & 1,290 \\
\hline Extension (Nyanza-Lac) & 41 & 31 & 14 & 0 & 0 \\
\hline Moso & 832 & 2,244 & 1,838 & 1,730 & 1,300 \\
\hline Number of farmers & 17,409 & 20,904 & 14,119 & 14,897 & 17,000 \\
\hline Production of seed cotton (tons) & 4,915 & 4,593 & 2,605 & 2,381 & 3,300 \\
\hline First quality & 4,811 & 4,553 & 2,526 & 2,310 & 3,200 \\
\hline Second quality & 104 & 40 & 79 & 71 & 100 \\
\hline Yield (kilograms per hectare) & 810 & 745 & 640 & 689 & 918 \\
\hline Ginning coefficient (percent) & 40 & 41 & 40 & 41 & 43 \\
\hline Production of cotton fiber (tons) & 1,967 & 1,881 & 1,045 & 972 & 1,400 \\
\hline First quality & 1,926 & 1,864 & 1,029 & 945 & 1,360 \\
\hline Second quality & 41 & 17 & 16 & 29 & 40 \\
\hline Production of seeds (tons) & 2,847 & 2,629 & 1,504 & 1,571 & 1,815 \\
\hline \multicolumn{6}{|l|}{ Consumption and exports (tons) } \\
\hline Domestic consumption of cotton fiber & 946 & 872 & 1,034 & 692 & 1,320 \\
\hline First quality & 845 & 857 & 957 & 677 & 1,280 \\
\hline Second quality & 101 & 15 & 77 & 15 & 40 \\
\hline Exports of cotton fiber & 3,665 & 893 & 151 & 0 & 200 \\
\hline First quality & 3,665 & 893 & 151 & 0 & 200 \\
\hline Second quality & 0 & 0 & 0 & 0 & 0 \\
\hline Stocks of cotton fiber (end of year) & 663 & 673 & 639 & 936 & 634 \\
\hline First quality & 610 & 657 & 639 & 918 & 616 \\
\hline Second quality & 53 & 16 & 0 & 18 & 18 \\
\hline \multicolumn{6}{|l|}{ Consumption of seeds } \\
\hline Oil factory (RAFINA) & 2,423 & 2,278 & 1,244 & 1,040 & 1,438 \\
\hline Seeds & 374 & 285 & 220 & 264 & 329 \\
\hline Animal feed & 50 & 66 & 40 & 67 & 48 \\
\hline \multicolumn{6}{|l|}{ Prices (Bunundi francs per kilogram) } \\
\hline \multicolumn{6}{|l|}{ Local price of cotton fiber } \\
\hline First quality & 349 & 452 & 498 & 520 & 580 \\
\hline Second quality & 212 & 278 & 400 & 410 & 450 \\
\hline \multicolumn{6}{|l|}{ Export price of cotton fiber $2 /$} \\
\hline First quality & 372 & 496 & 537 & $\ldots$ & 600 \\
\hline Second quality & $\ldots$ & $\ldots$ & $\ldots$ & $\cdots$ & $\ldots$ \\
\hline \multicolumn{6}{|l|}{ Producer price of seed cotton } \\
\hline First quality & 60 & 65 & 75 & 85 & 95 \\
\hline Second quality & 30 & 35 & 45 & 75 & 85 \\
\hline \multicolumn{6}{|l|}{ Equivalent producer price of cotton fiber } \\
\hline First quality & 150 & 160 & 187 & 213 & 288 \\
\hline Second quality & 74 & 88 & 112 & 188 & 213 \\
\hline Sale price of seeds to RAFINA & 14 & 14 & 15 & 15 & 25 \\
\hline \multicolumn{6}{|c|}{ Memorandum items: } \\
\hline \multicolumn{6}{|c|}{ Producer price/export price of cotton fiber } \\
\hline \multicolumn{6}{|l|}{ Real producer price of seed cotton } \\
\hline (first quality) $3 /$ & 272.5 & 297.4 & 257.5 & 205.0 & 195.5 \\
\hline
\end{tabular}

Sources: Compagnie de Gérance Cotonnière (COGERCO); and Complexe Textile de Bujumbura (COTEBU).

1/ Based on contracts signed by COTEBU.

2/ Prices are f.o.b. Dar es Salaam or Mombasa; since 1984, cotton has been exported at ex factory prices.

3/ Deflated by the consumer price index (January 1991=100). 
Table 9. Burundi: Tea Production, Exports, Stocks, and Prices, 1994-98

(In units indicated)

\begin{tabular}{|c|c|c|c|c|c|}
\hline & 1994 & 1995 & 1996 & $\begin{array}{r}1997 \\
\text { Est. }\end{array}$ & $\begin{array}{l}1998 \\
\text { Proj. }\end{array}$ \\
\hline \multicolumn{6}{|l|}{ Production } \\
\hline Area planted (hectares) & 7,829 & 7,916 & 7,916 & 7,916 & 7,916 \\
\hline Family holdings (hectares) & 5,923 & 5,940 & 5,940 & 5,940 & 5,940 \\
\hline Industrial blocks (hectares) & 1,976 & 1,976 & 1,976 & 1,976 & 1,976 \\
\hline Wastage factor (percent) & 5 & 5 & 5 & 5 & 5 \\
\hline Production of green leaves (tons) & 33,137 & 35,254 & 28,100 & 20,720 & 31,200 \\
\hline Yield (kilograms per hectare) & 4,233 & 4,454 & 3,550 & 2,617 & 3,941 \\
\hline Production of dry green leaves (tons) & 6,864 & 6,993 & 5,715 & 4,170 & 6,500 \\
\hline Teza & 1,647 & 1,716 & 998 & 376 & 1,354 \\
\hline Rwegura & 2,313 & 2,033 & 1,761 & 1,583 & 1,895 \\
\hline Tora & 1,052 & 1,192 & 1,128 & 949 & 1,263 \\
\hline Ijenda & 1,480 & 1,673 & 1,712 & 1,138 & 1,624 \\
\hline Buhoro & 372 & 379 & 116 & 124 & 364 \\
\hline $\begin{array}{l}\text { Exports (tons) } 1 / \\
\text { Of which }\end{array}$ & $5 ; 575$ & 6,795 & 3,412 & 6,330 & 6,500 \\
\hline Private sales & 1,376 & 1,480 & 987 & 2,037 & 5,200 \\
\hline Stocks (end of period; tons) & 2,660 & 2,852 & 5,150 & 2,986 & 670 \\
\hline \multicolumn{6}{|l|}{$\begin{array}{l}\text { Prices (Burundi francs per kilogram) } \\
\text { Producer price }\end{array}$} \\
\hline Planters & 26 & 33 & 33 & 33 & 33 \\
\hline Pickers (industrial blocks) & 8 & 9 & 9 & 9 & 9 \\
\hline Average unit export price $2 /$ & 452 & 408 & 504 & 700 & 850 \\
\hline Commercial freight 3 / & 85 & 90 & 120 & 250 & 300 \\
\hline Export price (f.o.b. Bujumbura) & 367 & 318 & 384 & 450 & 550 \\
\hline
\end{tabular}

Source: Office du Thé du Burundi (OTB).

1/ Quantities refer to tea leaving Bujumbura.

$2 /$ Calculated on the basis of sales contracts or auction prices.

3/ Transport and other commercial costs. 
$-15-$

Table 10. Burundi: Production of the Manufacturing Industries, 1994-98

\begin{tabular}{|c|c|c|c|c|c|c|}
\hline \multirow[t]{2}{*}{ Product } & \multirow[t]{2}{*}{ Unit of Measurement } & \multirow[t]{2}{*}{1994} & \multirow[t]{2}{*}{1995} & \multirow[t]{2}{*}{1996} & 1997 & $19981 /$ \\
\hline & & & & & \multicolumn{2}{|c|}{ Est. } \\
\hline Beer & Hectoliters & $1,382,670$ & $1,404,212$ & $1,227,921$ & $1,161,232$ & 500,380 \\
\hline Primus & Hectoliters & $1,187,058$ & $1,148,948$ & 997,188 & 983,683 & 431,305 \\
\hline Amstel & Hectoliters & 195,612 & 255,264 & 230,733 & 177,549 & 69,075 \\
\hline Carbonated beverages & Hectoliters & 201,400 & 219,746 & 179,079 & 146,580 & 62,550 \\
\hline Flour & Tons & 669 & 472 & 367 & $-\ldots$ & - \\
\hline Biscuits & Tons & 4 & $\ldots$ & $\ldots$ & $\ldots$ & $\ldots$ \\
\hline Milk & Millions of liters & 1 & 1 & 1 & 0.30 & 0.12 \\
\hline Dairy products & Kilograms & 40,132 & $\ldots$ & $\ldots$ & $\ldots$ & ... \\
\hline Cottonseed oil & Liters & 279,900 & 211,450 & 234,600 & 199,715 & 7,900 \\
\hline Sugar & Tons & 12,268 & 15,300 & 17,756 & 19,582 & $\ldots$ \\
\hline Animal feed & Tons & 2,443 & 1,909 & 2,339 & 1,591 & 422 \\
\hline Cigarettes 21 & Cartons & 58,458 & 52,248 & 44,986 & 37,714 & 15,041 \\
\hline Paint & Tons & 591 & 529 & 407 & 369 & 160 \\
\hline Insecticides & Tons & 3,885 & 2,406 & 2,418 & 2,374 & 151 \\
\hline Oxygen & Cubic meters & 33,636 & 29,119 & 26,195 & 30,049 & 12,554 \\
\hline Acetylene & Kilograms & 4,705 & 3,279 & 1,871 & $\ldots$ & \\
\hline Polyethylene & Kilograms & 292,847 & 267,247 & 240,205 & 181,834 & 63,794 \\
\hline Mattresses & Units & 50,225 & 29,505 & 29,587 & 23,984 & 14,757 \\
\hline Plastic cases & Units & 48,904 & 50,409 & 49,093 & 21,276 & 4,896 \\
\hline Household soap & Tons & 5,292 & 5,262 & 3,111 & 2,431 & 1,445 \\
\hline Toilet soap & Tons & 409 & 306 & 259 & 332 & 106 \\
\hline Pharmaceutical products & Millions of Burundi franes & 272 & 243 & 294 & 328 & 198 \\
\hline Matches 3/ & Cartons & 13,374 & 11,544 & 256 & $\ldots$ & $\ldots$ \\
\hline Bottles & Tons & 5,106 & 3,704 & 2,463 & 2,061 & $\ldots$ \\
\hline Blankets & Units & 248,438 & 137,708 & 116,245 & 21,727 & 7,485 \\
\hline Fabrics & Millions of square meters & 5 & 4 & 5 & 5 & 2 \\
\hline Shoes & Pairs & 74,890 & 10,000 & $\ldots$ & $\ldots$ & $\ldots$ \\
\hline P.V.C. tubes & Tons & 188 & 214 & 113 & 68 & 38 \\
\hline Cement & Tons & $\ldots$ & $\ldots$ & $\ldots$ & $\ldots$ & $\ldots$ \\
\hline Fibro-cement products & Tons & 799 & 566 & 249 & 686 & 337 \\
\hline Corrugated metal sheets & Tons & 564 & 86 & 0 & 486 & 425 \\
\hline Steel rods & Tons & 307 & 285 & 118 & 146 & $\ldots$ \\
\hline Toilet paper & Tons & 14 & 18 & 86 & 46 & $\ldots$ \\
\hline Bottle caps & Millions of units & 283 & 274 & 272 & 141 & 136 \\
\hline Batteries 4/ & Cartons & 28,867 & $\ldots$ & 14,453 & ... & ... \\
\hline Cardboard boxes & Tons & 287 & 154 & ... & $\ldots$ & $\ldots$ \\
\hline
\end{tabular}

Source: Bank of the Republic of Burundi.

1/ From January to May 1998.

2/ Cartons of 10,000 cigarettes except Kiyago, which is in cartons of 5,000 cigarettes.

$3 /$ Cartons of 1,000 boxes.

4/ Cartons of 240 batteries. 
Table 11. Burundi: Energy Production, Imports, and Consumption, 1994-98

\begin{tabular}{|c|c|c|c|c|c|}
\hline & 1994 & 1995 & 1996 & 1997 & $19981 /$ \\
\hline & & & & & \\
\hline & \multicolumn{5}{|c|}{ (In units indicated) } \\
\hline \multicolumn{6}{|l|}{ Electricity (in thousands of kilowatt-hours) } \\
\hline Production & 82,675 & 97,199 & 99,262 & 87,804 & 50,340 \\
\hline Imports & 64,064 & 31,001 & 3,854 & 42,860 & 15,317 \\
\hline Ruzizil & 16,596 & 11,261 & 3,819 & 18,846 & 8,375 \\
\hline Ruzizi II & 47,468 & 19,740 & 35 & 24,014 & 6,942 \\
\hline Consumption & 124,800 & 97,476 & 92,686 & 92,200 & 52,948 \\
\hline \multicolumn{6}{|l|}{ Of which } \\
\hline Industry & 38,109 & 43,400 & 45,400 & 44,500 & ... \\
\hline Domestic rate (Burundi francs per kilowatt-hour) & 22 & 22 & 22 & 22 & 22 \\
\hline \multicolumn{6}{|l|}{ Petroleum products } \\
\hline Consumption (tons) & 82,469 & 70,118 & 48,197 & 39,888 & 24,983 \\
\hline Imports (tons) & 79,175 & 71,183 & 45,785 & 48,651 & 22,039 \\
\hline Stocks at end of period (tons) & 4,735 & 5,800 & $3, \mathbf{3 8 8}$ & 12,151 & 9,207 \\
\hline \multicolumn{6}{|l|}{ Unit value of imports } \\
\hline \multirow{2}{*}{\multicolumn{6}{|c|}{ Import value }} \\
\hline & & & & & \\
\hline \multirow[t]{2}{*}{ (Millions of Burundi francs) 2/ } & 7,328 & 6,645 & 5,511 & 4,863 & 4,192 \\
\hline & \multicolumn{5}{|c|}{ (Annual changes in percent) } \\
\hline \multicolumn{6}{|l|}{ Electricity } \\
\hline Production & -18 & 18 & 2 & -12 & $\cdot \ldots$ \\
\hline Imports & 34 & -52 & -88 & 1,012 & $\ldots$ \\
\hline $\begin{array}{l}\text { Consumption } \\
\text { Of which }\end{array}$ & 0 & -22 & -5 & -1 & $\ldots$ \\
\hline $\begin{array}{l}\text { Of which } \\
\text { Industry }\end{array}$ & \multicolumn{4}{|c|}{ Of which } & ... \\
\hline \multicolumn{6}{|l|}{ Petroleum products } \\
\hline Consumption & 29 & -15 & -31 & -17 & ... \\
\hline Imports & 21 & .10 & -36 & 6 & $\ldots$ \\
\hline Stocks at end of period & -37 & 22 & -42 & 259 & ... \\
\hline Unit value of imports (in Burundi francs) & 2 & 1 & 29 & -17 & ... \\
\hline Import value (in Burundi francs) & 27 & -9 & -17 & -12 & $\ldots$ \\
\hline
\end{tabular}

Sources: Régie de Distribution d'Eau et d'Electricité (REGIDESO); and Société d'Entreposage de Produits Petroliers (SEP).

1/ From January to June.

2/ Customs data. 
Table 12. Burundi: Retail Prices of Petroleum Products, 1994-98

(Burundi francs per liter)

\begin{tabular}{lccccc}
\hline & 1994 & $19951 /$ & 1996 & 1997 & $\begin{array}{r}1998 \\
\text { End- } \\
\text { October }\end{array}$ \\
\hline Super gasoline 2/ & 140 & 160 & 350 & 350 & 350 \\
Kerosene 3/ & 130 & 142 & 165 & 165 & 165 \\
Diesel fuel 4/ & 130 & 145 & 320 & 320 & 320 \\
\hline
\end{tabular}

Source: Ministry of Commerce and Industry.

1/ As of December, 23, 1995.

2/ The price of gasoline for 1996 was set at FBu 190 per liter on July 15, 1996; FBu 270 per liter on September 20, 1996; and FBu 350 per liter on November 7, 1996.

3/ The price of kerosene for 1996 was set at FBu 165 per liter on July 15,1996.

4/ The price of diesel fuel for 1996 was set at FBu 170 per liter on July 15, 1996; FBu 240 per liter on September 20, 1996; and FBu 350 per liter on November 7, 1996. 
$-18-$

Table 13. Burundi: Minimum Wages, 1994-98

\begin{tabular}{|c|c|c|c|c|c|}
\hline & 1994 & 1995 & 1996 & 1997 & $19981 /$ \\
\hline & \multicolumn{5}{|c|}{ (In Burundi francs per day) } \\
\hline Urban areas $2 /$ & 160 & 160 & 160 & 160 & 160 \\
\hline \multirow[t]{2}{*}{ Rest of the country } & 105 & 105 & 105 & 105 & 105 \\
\hline & \multicolumn{5}{|c|}{ (In real terms) $3 /$} \\
\hline Urban areas $2 /$ & 124.8 & 104.6 & 82.7 & 63.1 & 53.9 \\
\hline \multirow[t]{2}{*}{ Rest of the country } & 81.9 & 68.6 & 54.3 & 41.4 & 35.4 \\
\hline & \multicolumn{5}{|c|}{ (In U.S. dollars) 4/ } \\
\hline Urban areas $2 /$ & 0.63 & 0.64 & 0.53 & 0.45 & 0.37 \\
\hline Rest of the country & 0.42 & 0.42 & 0.35 & 0.30 & 0.24 \\
\hline \multicolumn{6}{|l|}{ Memorandum items: } \\
\hline \multicolumn{6}{|l|}{ Consumer price index } \\
\hline (January 1991=100) & 128.2 & 153.0 & 193.4. & 253.6 & 296.6 \\
\hline Exchange rate (FBu/US\$; period average) & 252.7 & 249.8 & 302.8 & 352.4 & 435.2 \\
\hline
\end{tabular}

Source: Ministry of Labor and Social Affairs.

1/ As of April 30, 1998

2/ Bujumbura and Gitega.

3/ Deflated by the consumer price index (January 1991=100).

4/ Converted using period-average exchange rates. 
Table 14. Burundi: Consumer Price Index for Households in Bujumbura, 1994-98

(January 1991=100)

\begin{tabular}{|c|c|c|c|c|c|c|c|c|c|c|}
\hline & Food & Clothing & $\begin{array}{l}\text { Housing, } \\
\text { Heating, } \\
\text { and Light }\end{array}$ & Furniture & Health & Transport & $\begin{array}{r}\text { Culture, } \\
\text { Education, } \\
\text { and Leisure }\end{array}$ & Other & $\begin{array}{r}\text { General } \\
\text { Index }\end{array}$ & $\begin{array}{r}\text { Change of } \\
\text { General } \\
\text { Index 1/ }\end{array}$ \\
\hline Basket weight & 52.6 & 5.4 & 27.4 & 49.1 & 0.7 & 5.4 & 1.9 & 1.7 & 100.0 & \\
\hline 1994 & 133.7 & 136.0 & 119.2 & 140.9 & 137.4 & 101.4 & 123.4 & 127.4 & 128.2 & 14.9 \\
\hline 1995 & 160.4 & 157.0 & 148.0 & 153.7 & 166.8 & 102.0 & 134.2 & 153.8 & 153.0 & 19.4 \\
\hline Q1 & 153.4 & 144.5 & 128.4 & 145.5 & 165.4 & 102.0 & 129.8 & 147.2 & 142.7 & 18.7 \\
\hline Q2 & 163.8 & 154.6 & 138.6 & 152.2 & 165.4 & 102.0 & 132.0 & 152.2 & 151.9 & 21.1 \\
\hline Q3 & 161.9 & 160.7 & 159.7 & 159.0 & 165.4 & 102.0 & 135.5 & 155.2 & 157.4 & 18.7 \\
\hline $\mathrm{Q} 4$ & 162.5 & 168.2 & 165.3 & 158.1 & 170.8 & 102.0 & 139.4 & 160.7 & 159.9 & 19.0 \\
\hline 1996 & 199.0 & 199.3 & 192.1 & 170.3 & 210.2 & 176.4 & 155.5 & 172.7 & 193.4 & 26.4 \\
\hline Q1 & 174.2 & 178.3 & 182.7 & 161.7 & 177.3 & 162.2 & 143.4 & 163.1 & 174.8 & 22.4 \\
\hline Q2 & 186.4 & 183.2 & 182.8 & 165.0 & 195.2 & 162.2 & 143.8 & 168.4 & 181.6 & 19.6 \\
\hline Q3 & 208.0 & 194.3 & 197.5 & 170.8 & 228.1 & 180.0 & 143.5 & 170.4 & 199.7 & 26.9 \\
\hline $\mathrm{Q} 4$ & 227.4 & 241.4 & 205.3 & 183.5 & 240.3 & 201.4 & 191.2 & 188.9 & 217.4 & 36.0 \\
\hline 1997 & 268.7 & 308.7 & 235.4 & 201.6 & 241.0 & 229.1 & 200.9 & 210.0 & 253.6 & 31.1 \\
\hline Q1 & 246.4 & 276.6 & 214.5 & 187.1 & 223.3 & 206.7 & 194.5 & 196.1 & 232.1 & 32.8 \\
\hline Q2 & 270.2 & 334.6 & 219.7 & 199.4 & 251.4 & 216.3 & 194.9 & 203.3 & 250.6 & 38.0 \\
\hline Q3 & 269.4 & 312.9 & 234.9 & 205.5 & 248.0 & 262.4 & 191.9 & 211.7 & 256.1 & 28.2 \\
\hline Q4 & 288.6 & 310.6 & 272.4 & 214.4 & 241.4 & 231.0 & 222.3 & 229.1 & 275.5 & 26.7 \\
\hline $19982 /$ & $\ldots$ & $\ldots$ & $\ldots$ & $\ldots$ & $\ldots$ & $\ldots$ & $\ldots$ & $\ldots$ & 296.6 & 17.0 \\
\hline Q1 & 313.4 & 326.3 & 278.0 & 217.7 & 243.8 & 216.9 & 229.0 & 228.1 & 290.3 & 25.1 \\
\hline January & 328.6 & 319.6 & 274.9 & 211.2 & 261.4 & 216.8 & 229.9 & 230.6 & 297.1 & 30.9 \\
\hline February & 298.2 & 329.1 & 275.5 & 222.4 & 261.4 & 216.8 & 236.6 & 226.2 & 282.5 & 22.1 \\
\hline March & 313.4 & 330.1 & 283.5 & 219.4 & 208.7 & 217.0 & 220.5 & 227.5 & 291.2 & 22.4 \\
\hline Q2 & 318.0 & 329.0 & 276.8 & 223.6 & 214.5 & 217.1 & 215.7 & 227.9 & 291.9 & 16.5 \\
\hline April & 322.8 & 331.8 & 281.7 & 225.3 & 215.3 & 217.0 & 218.2 & 227.5 & 296.0 & 23.1 \\
\hline May & 323.1 & 325.0 & 276.8 & 224.7 & 215.3 & 217.1 & 214.4 & 228.4 & 294.4 & 13.1 \\
\hline June & 308.0 & 330.1 & 271.8 & 220.8 & 213.0 & 217.1 & 214.4 & 227.7 & 285.4 & 13.7 \\
\hline
\end{tabular}

Sources: Institut de Statistiques et des Etudes Economiques du Burundi; and Fund staff projections.

1/ Average percent changes for annual data; year-on-year changes for quarterly and monthly data.

2/ Projection. 
Table 15. Burundi: Central Government Financial Operations, 1994-98

\begin{tabular}{|c|c|c|c|c|c|}
\hline & 1994 & 1995 & 1996 & $\begin{array}{r}1997 \\
\text { Est. }\end{array}$ & $\begin{array}{c}1998 \\
\text { Proj. }\end{array}$ \\
\hline & \multicolumn{5}{|c|}{ (In billions of Burundi francs) } \\
\hline Revenue and grants & 47.9 & 53.2 & 48.5 & 56.6 & 64.5 \\
\hline Revenue & 42.2 & 44.1 & 42.3 & 46.3 & 53.2 \\
\hline Tax revenue & 38.9 & 41.5 & 35.4 & 43.7 & 50.3 \\
\hline Income tax & 8.4 & 9.4 & 10.6 & 11.9 & 12.0 \\
\hline Taxes on goods and services & 17.7 & 19.0 & 16.9 & 22.0 & 23.0 \\
\hline Taxes on international trade & 12.5 & 12.8 & 7.5 & 8.9 & 15.2 \\
\hline Other tax revenue & 0.3 & 0.3 & 0.4 & 0.9 & 0.1 \\
\hline Nontax revenue & 3.3 & 2.7 & 7.0 & 2.6 & 2.9 \\
\hline Grants & 5.7 & 9.1 & 6.1 & 10.3 & 11.3 \\
\hline Current grants & 1.1 & 3.1 & 0.1 & 6.1 & 6.1 \\
\hline Capital grants & 4.6 & 6.0 & 6.0 & 4.2 & 5.2 \\
\hline Expenditure and net lending & 57.9 & 65.3 & 76.0 & 74.9 & 82.5 \\
\hline Current expenditure & 39.1 & 45.1 & 48.1 & 61.4 & 61.2 \\
\hline Salaries & 17.4 & 18.1 & 21.5 & 24.1 & 25.6 \\
\hline Goods and services & 10.4 & 12.6 & 13.0 & 18.3 & 21.4 \\
\hline Transfers and subsidies & 5.8 & 7.0 & 5.9 & 5.7 & 7.6 \\
\hline Interest payments & 3.6 & 4.5 & 5.1 & 6.7 & 6.6 \\
\hline Domestic & 0.7 & 1.0 & 1.0 & 2.8 & 2.2 \\
\hline Foreign & 2.9 & 3.5 & 4.1 & 3.9 & 4.4 \\
\hline Extrabudgetary/exceptional expenditure & 1.9 & 2.9 & 2.6 & 6.6 & 0.0 \\
\hline Capital expenditure & 21.0 & 20.7 & 28.4 & 16.9 & 21.3 \\
\hline Domestic resources & 4.1 & 4.6 & 8.5 & 3.5 & 3.0 \\
\hline Project lending & 12.3 & 10.1 & 13.9 & 9.2 & 13.1 \\
\hline Capital grants & 4.6 & 6.0 & 6.0 & 4.2 & 5.2 \\
\hline Net lending & -2.1 & -0.4 & -0.5 & -3.4 & 0.0 \\
\hline Current primary balance & 4.5 & 1.8 & -6.6 & -5.3 & -4.4 \\
\hline \multicolumn{6}{|l|}{ Overall balance (commitment basis) } \\
\hline Including grants & -10.1 & -12.2 & -27.6 & -18.3 & -18.0 \\
\hline Excluding grants & -15.7 & -21.2 & -33.7 & .28 .6 & -29.3 \\
\hline Change in arrears (reduction -) & 0.3 & 4.6 & 10.1 & 6.0 & 10.9 \\
\hline External & 1.0 & 2.3 & 7.5 & 5.5 & 7.1 \\
\hline Domestic & -0.7 & 2.3 & 2.6 & 0.5 & 3.8 \\
\hline Overall balance (cash basis) & -9.7 & -7.6 & -17.5 & -12.3 & -7.1 \\
\hline Financing & 9.7 & 7.6 & 17.5 & 12.3 & 7.1 \\
\hline Foreign & 6.3 & 3.6 & 2.2 & 1.3 & 3.5 \\
\hline Drawings & 12.8 & 12.4 & 13.9 & 9.2 & 13.1 \\
\hline Amortization & -6.5 & .8 .8 & -11.7 & -7.9 & -9.6 \\
\hline Domestic & 3.4 & 4.0 & 15.3 & 11.0 & 3.6 \\
\hline Banking sector & 3.7 & -2.9 & 11.1 & 11.0 & 3.6 \\
\hline Nonbank sector & -0.3 & 6.9 & 4.2 & 0.0 & 0.0 \\
\hline \multirow[t]{2}{*}{ Financing gap (deficit + ) } & 0.0 & 0.0 & 0.0 & 0.0 & 0.0 \\
\hline & \multicolumn{5}{|c|}{ (In percent of GDP, unless otherwise indicated) } \\
\hline Memorandum items: & & & & & \\
\hline Revenue, excluding grants & 18.1 & 17.7 & 15.5 & 13.7 & 12.5 \\
\hline Current expenditure & 16.8 & 18.0 & 17.6 & 18.2 & 14.4 \\
\hline Current primary balance (excluding grants) & 1.9 & 0.7 & -2.4 & -1.6 & -1.0 \\
\hline Capital expenditure & 9.0 & 8.3 & 10.4 & 5.0 & 5.0 \\
\hline Total expenditure and net lending & 24.8 & 26.1 & 27.9 & 22.2 & 19.4 \\
\hline \multicolumn{6}{|l|}{ Overall balance (commitment basis) } \\
\hline Including grants & -4.3 & -4.9 & -10.1 & -5.4 & -4.2 \\
\hline Excluding grants & -6.7 & -8.5 & -12.4 & -8.5 & -6.9 \\
\hline Overall balance (cash basis) & -4.2 & -3.0 & -6.4 & -3.7 & -1.7 \\
\hline Domestic financing & 1.5 & 1.6 & 5.6 & 3.3 & 0.8 \\
\hline Foreign financing & 2.7 & 1.4 & 0.8 & 0.4 & 0.8 \\
\hline GDP (in billions of Burundi francs) & 233.3 & 249.9 & 272.6 & 337.3 & 425.1 \\
\hline
\end{tabular}

Sources: Burundi authorities; and Fund staff estimates and projections. 
Table 16. Burundi: Central Government Revenue, 1994-98

\begin{tabular}{|c|c|c|c|c|c|}
\hline & 1994 & 1995 & 1996 & $\begin{array}{r}1997 \\
\text { Est. }\end{array}$ & $\begin{array}{c}1998 \\
\text { Proj. }\end{array}$ \\
\hline & \multicolumn{5}{|c|}{ (In billions of Burundi francs) } \\
\hline Tax revenue & 38.9 & 41.5 & 35.4 & 43.7 & 50.3 \\
\hline Tax on income and profits & 8.4 & 9.4 & 10.6 & 11.9 & 12.0 \\
\hline Individual & 4.1 & 3.9 & 4.9 & 5.7 & 5.9 \\
\hline Corporate & 3.9 & 4.9 & 5.4 & 5.7 & 5.7 \\
\hline Other & 0.4 & 0.5 & 0.3 & 0.5 & 0.4 \\
\hline Tax on property & 0.2 & 0.2 & 0.2 & 0.0 & 0.0 \\
\hline Taxes on goods and services & 17.7 & 19.0 & 16.9 & 22.0 & 23.0 \\
\hline Transaction tax & 6.7 & 7.1 & 6.1 & 8.6 & 8.9 \\
\hline Excise tax & 10.7 & 11.9 & 10.8 & 13.1 & 13.7 \\
\hline Other taxes on goods and services & 0.4 & 0.0 & 0.0 & 0.3 & 0.4 \\
\hline Taxes on international trade & 12.5 & 12.8 & 7.5 & 8.9 & 15.2 \\
\hline Import duties & 6.9 & 7.7 & 5.9 & 5.8 & 9.9 \\
\hline Export duties & 5.4 & 4.9 & 1.3 & 3.0 & 5.3 \\
\hline Of which: coffee & 5.3 & 4.7 & 1.2 & 0.6 & 5.2 \\
\hline Other & 0.1 & 0.1 & 0.3 & 0.1 & 0.3 \\
\hline Other tax revenue & 0.1 & 0.1 & 0.2 & 0.9 & 0.1 \\
\hline Nontax revenue & 3.3 & 2.7 & 7.0 & 2.6 & 2.9 \\
\hline Dividends and profits & 2.6 & 1.9 & 6.0 & 1.3 & 1.5 \\
\hline Administrative receipts & 0.5 & 0.3 & 0.4 & 0.7 & 0.8 \\
\hline Other nontax revenue & 0.2 & 0.5 & 0.6 & 0.6 & 0.6 \\
\hline \multirow[t]{2}{*}{ Total revenue } & 42.2 & 44.1 & 42.3 & 46.3 & 53.2 \\
\hline & \multicolumn{5}{|c|}{ (In percent of total revenue) } \\
\hline Tax revenue & 92.2 & 94.0 & 83.6 & 94.4 & 94.5 \\
\hline Tax on income and profits & 19.9 & 21.2 & 25.1 & 25.7 & 22.6 \\
\hline Tax on property & 0.5 & 0.5 & 0.5 & 0.0 & 0.0 \\
\hline Taxes on goods and services & 42.0 & 43.1 & 39.9 & 47.5 & 43.2 \\
\hline Taxes on international trade & 29.6 & 28.9 & 17.7 & 19.2 & 28.6 \\
\hline Other tax revenue & 0.2 & 0.2 & 0.5 & 1.9 & 0.2 \\
\hline \multirow[t]{2}{*}{ Nontax revenue } & 7.8 & 6.0 & 16.4 & 5.6 & 5.5 \\
\hline & \multicolumn{5}{|c|}{ (In percent of GDP) } \\
\hline Tax revenue & 16.7 & 16.6 & 13.0 & 13.0 & 11.8 \\
\hline Tax on income and profits & 3.6 & 3.7 & 3.9 & 3.5 & 2.8 \\
\hline Tax on property & 0.1 & 0.1 & 0.1 & 0.0 & 0.0 \\
\hline Taxes on goods and services & 7.6 & 7.6 & 6.2 & 6.5 & 5.4 \\
\hline Taxes on international trade & 5.4 & 5.1 & 2.7 & 2.6 & 3.6 \\
\hline Other tax revenue & 0.0 & 0.0 & 0.1 & 0.3 & 0.0 \\
\hline \multirow[t]{2}{*}{ Nontax revenue } & 1.4 & 1.1 & 2.6 & 0.8 & 0.7 \\
\hline & \multicolumn{5}{|c|}{ (Annual percentage change) } \\
\hline Tax revenue & 12.5 & 6.5 & -14.6 & 23.5 & 15.1 \\
\hline Tax on income and profits & -20.9 & 11.4 & 13.4 & 12.1 & 0.8 \\
\hline Tax on property & 21.4 & -1.8 & 0.4 & -100.0 & $\ldots$ \\
\hline Taxes on goods and services & 15.9 & 7.2 & -11.1 & 30.3 & 4.5 \\
\hline Taxes on international trade & 50.3 & 2.3 & .41 .4 & 19.0 & 70.8 \\
\hline Other tax revenue & -47.1 & 0.0 & 100.0 & 350.0 & -90.0 \\
\hline Nontax revenue & -36.6 & -19.3 & 162.2 & -62.6 & 11.5 \\
\hline \multicolumn{6}{|l|}{ Memorandum item: } \\
\hline Nominal GDP (in billions of Burundi francs) & 233.3 & 249.9 & 272.6 & 337.3 & 425.1 \\
\hline
\end{tabular}

Sources: Burundi authorities; and Fund staff estimates and projections. 
Table 17. Burundi: Import Duty Exemptions, 1994-98

(In millions of Burundi francs, unless otherwise specified)

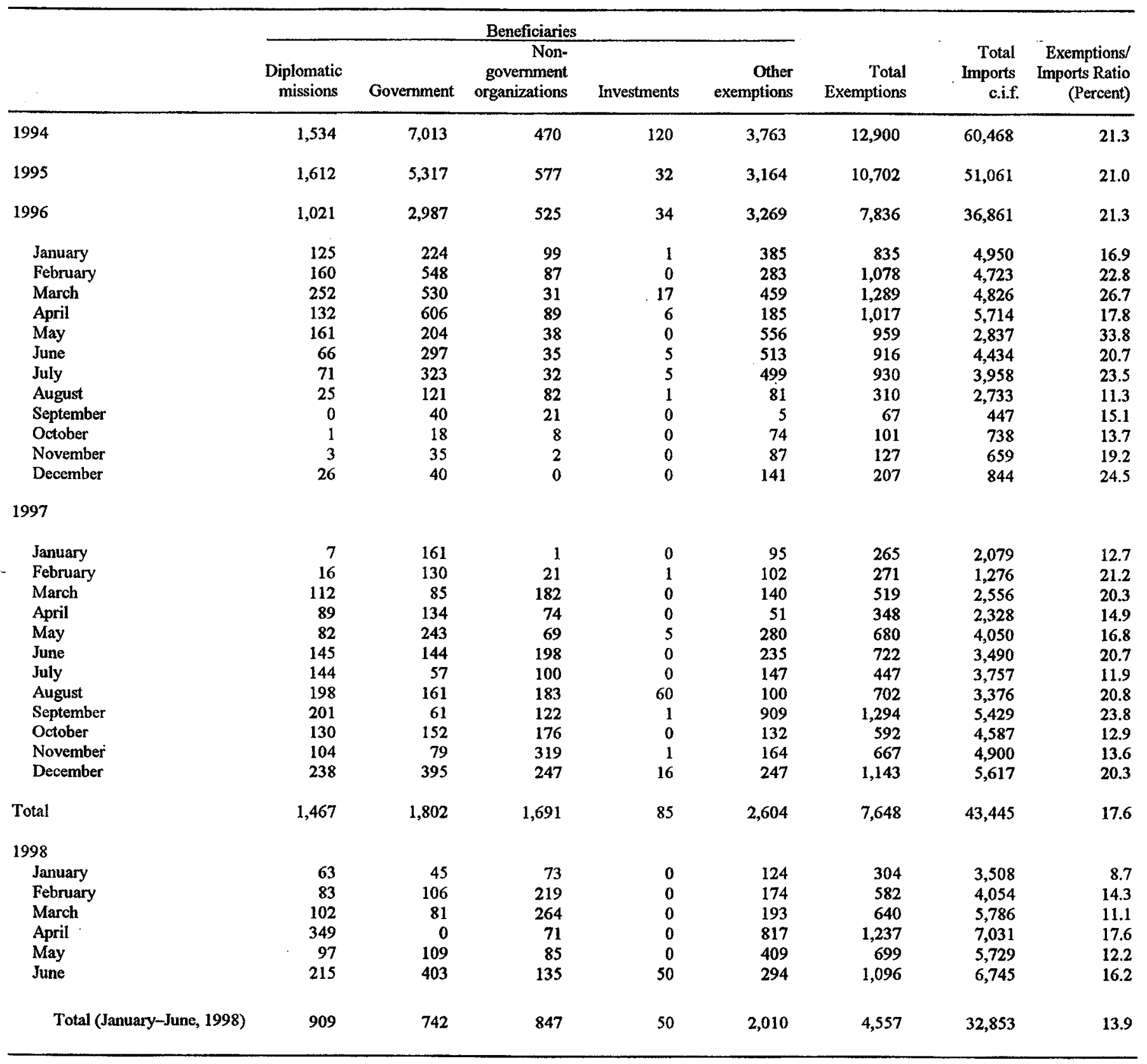

Source: Burundi authorities. 
Table 18. Burundi: Economic Classification of Government Expenditure, 1994-98

\begin{tabular}{|c|c|c|c|c|c|}
\hline & 1994 & 1995 & 1996 & $\begin{array}{r}1997 \\
\text { Est. }\end{array}$ & $\begin{array}{l}1998 \\
\text { Proj. }\end{array}$ \\
\hline & \multicolumn{5}{|c|}{ (In billions of Burundi francs) } \\
\hline Total expenditure and net lending & 57.9 & 65.3 & 76.0 & 74.9 & 82.5 \\
\hline Current expenditure & 39.1 & 45.1 & 48.1 & 61,4 & 61.2 \\
\hline Salaries & 17.4 & 18.1 & 21.5 & 24.1 & 25.6 \\
\hline Civilian & 11.5 & 11.5 & 13.3 & 12.9 & $\ldots$ \\
\hline Military & 5.9 & 6.6 & 8.2 & 11.2 & $\cdots$ \\
\hline Goods and services & 10.4 & 12.6 & 13.0 & 18.3 & 21.4 \\
\hline Civilian & 6.1 & 8.2 & 6.6 & 8.4 & $\ldots$ \\
\hline Military & 4.3 & 4.4 & 6.4 & 9.9 & $\cdots$ \\
\hline Transfers and subsidies & 5.8 & 7.0 & 5.9 & 5.7 & 7.6 \\
\hline Public administrations & 4.3 & 4.4 & 4.4 & 4.6 & 6.2 \\
\hline International organizations & 0.2 & 0.2 & 0.2 & 0.2 & 0.2 \\
\hline Households & 1.3 & 2.4 & 1.4 & 0.9 & 1.2 \\
\hline Interest payments & 3.6 & 4.5 & 5.1 & 6.7 & 6.6 \\
\hline Domestic & 0.7 & 1.0 & 1.0 & 2.8 & 2.2 \\
\hline Foreign & 2.9 & 3.5 & 4.1 & 3.9 & 4.4 \\
\hline Extrabudgetary/exceptional expenditure & 1.9 & 2.9 & 2.6 & 6.6 & 0.0 \\
\hline Capital expenditure & 21.0 & 20.7 & 28.4 & 16.9 & 21.3 \\
\hline Domestic resources & 4.1 & 4.6 & 8.5 & 3.5 & 3.0 \\
\hline Project lending & 12.3 & 10.1 & 13.9 & 9.2 & 13.1 \\
\hline Capital grants & 4.6 & 6.0 & 6.0 & 4.2 & 5.2 \\
\hline \multirow[t]{2}{*}{ Net lending } & -2.1 & -0.4 & -0.5 & .3 .4 & 0.0 \\
\hline & \multicolumn{5}{|c|}{ (In percent of total expenditure) } \\
\hline Total expenditure and net lending & 100.0 & 100.0 & 100.0 & 100.0 & 100.0 \\
\hline Current expenditure & 67.5 & 69.0 & 63.2 & 82.0 & 74.2 \\
\hline Salaries & 30.0 & 27.7 & 28.2 & 32.2 & 31.0 \\
\hline Of which: military & 10.1 & 10.1 & 10.8 & 14.9 & \\
\hline Goods and services & 18.0 & 19.3 & 17.1 & 24.4 & 25.9 \\
\hline Of which: military & 7.4 & 6.7 & 8.5 & 13.2 & \\
\hline Transfers and subsidies & 10.0 & 10.8 & 7.8 & 7.6 & 9.2 \\
\hline Public administrations & 7.5 & 6.7 & 5.8 & 6.1 & 7.5 \\
\hline International organizations & 0.4 & 0.3 & 0.2 & 0.3 & 0.2 \\
\hline Households & 2.2 & 3.7 & 1.8 & 1.1 & 1.5 \\
\hline Interest payments & 6.2 & 6.8 & 6.7 & 9.0 & 8.0 \\
\hline Domestic & 1.2 & 1.5 & 1.3 & 3.7 & 2.7 \\
\hline Foreign & 5.1 & 5.4 & 5.4 & 5.2 & 5.3 \\
\hline Extrabudgetary/exceptional expenditure & 3.3 & 4.4 & 3.4 & 8.8 & 0.0 \\
\hline Capital expenditure & 36.2 & 31.7 & 37.4 & 22.6 & 25.8 \\
\hline Domestic resources & 7.0 & 7.0 & 11.2 & 4.7 & 3.6 \\
\hline Project lending & 21.2 & 15.5 & 18.3 & 12.3 & 15.9 \\
\hline Capital grants & 7.9 & 9.2 & 7.9 & 5.6 & 6.3 \\
\hline \multirow[t]{2}{*}{ Net lending } & -3.7 & -0.6 & -0.6 & -4.5 & 0.0 \\
\hline & \multicolumn{5}{|c|}{ (In percent of GDP) } \\
\hline Total expenditure and net lending & 24.8 & 26.1 & 27.9 & 22.2 & 19.4 \\
\hline Current expenditure & 16.8 & 18.0 & 17.6 & 18.2 & 14.4 \\
\hline Salaries & 7.4 & 7.2 & 7.9 & 7.1 & 6.0 \\
\hline Interest payments & 1.6 & 1.8 & 1.9 & 2.0 & 1.6 \\
\hline Other current expenditure & 7.8 & 9.0 & 7.9 & 9.1 & 6.8 \\
\hline Capital expenditure & 9.0 & 8.3 & 10.4 & 5.0 & 5.0 \\
\hline \multicolumn{6}{|l|}{ Memorandum item: } \\
\hline Nominal GDP (in billions of Burundi francs) & 233.3 & 249.9 & 272.6 & 337.3 & 425.1 \\
\hline
\end{tabular}

Sources: Burundi authorities; and Fund staff estimates and projections. 
Table 19. Burundi: Expenditure on Health and Education, 1994-97

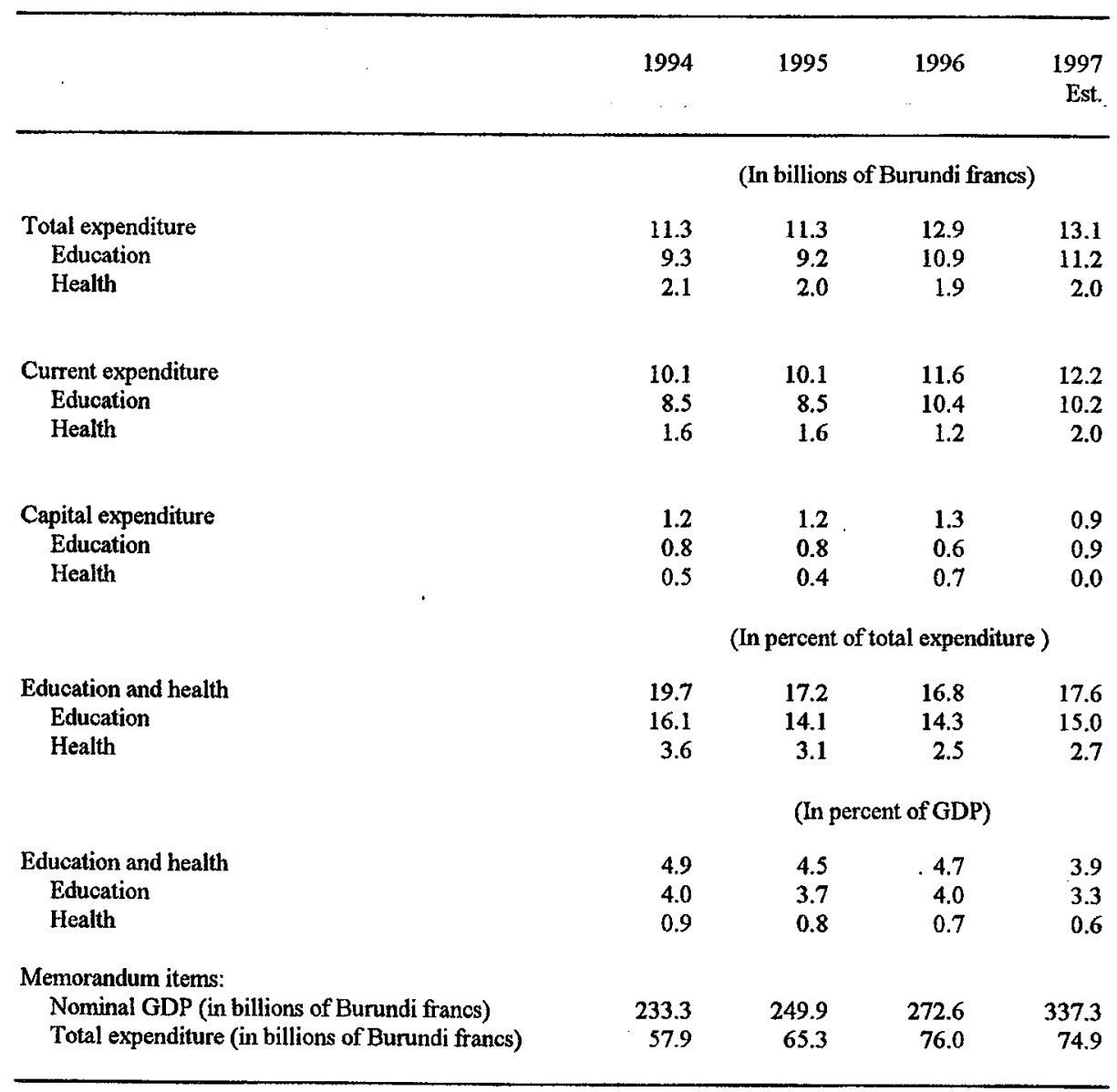

Sources: Burundi authorities; and Fund staff estimates. 
Table 20. Burundi: Military Expenditure, 1994-97

\begin{tabular}{|c|c|c|c|c|}
\hline & 1994 & 1995 & 1996 & $\begin{aligned} 1997 \\
\text { Est. }\end{aligned}$ \\
\hline & \multicolumn{4}{|c|}{$\begin{array}{l}\text { (In millions of Burundi francs, unless } \\
\text { otherwise specified) }\end{array}$} \\
\hline Salaries & 5,852 & 6,613 & 8,192 & 11,162 \\
\hline Goods and services & 4,274 & 4,398 & 6,438 & 9,868 \\
\hline Light equipment, food, clothing, and health care & 1,943 & 2,095 & 3,745 & 4,824 \\
\hline Equipment and maintenance $1 /$ & 1,574 & 2,005 & 2,440 & 4,425 \\
\hline Other & 758 & 298 & 254 & 619 \\
\hline \multirow[t]{2}{*}{ Total military expenditure } & 10,126 & 11,010 & 14,630 & 21,030 \\
\hline & \multicolumn{4}{|c|}{ (In percent) } \\
\hline Military salaries/total salaries & 33.7 & 36.5 & 38.2 & 46.4 \\
\hline on goods and services & 41.1 & 34.9 & 49.5 & 53.9 \\
\hline \multirow[t]{2}{*}{ Total military expenditure/total current expenditure } & 25.9 & 24.5 & 30.4 & 34.3 \\
\hline & \multicolumn{4}{|c|}{ (In percent of total expenditure and net lending) } \\
\hline Military salaries & 10.1 & 10.1 & 10.8 & 14.9 \\
\hline Military spending on goods and services & 7.4 & 6.7 & 8.5 & 13.3 \\
\hline \multirow[t]{2}{*}{ Total military expenditure } & 17.5 & 16.9 & 19.2 & 28.1 \\
\hline & \multicolumn{4}{|c|}{ (In percent of GDP) } \\
\hline Military salaries & 2.5 & 2.6 & 3.0 & 3.3 \\
\hline Military spending on goods and services & 1.2 & 1.1 & 2.4 & 2.9 \\
\hline Total military expenditure & 3.7 & 3.6 & 5.4 & 6.2 \\
\hline \multirow{2}{*}{\multicolumn{5}{|c|}{ Memorandum items: }} \\
\hline & & & & \\
\hline Nominal GDP & 233,320 & 249,865 & 272,582 & 337,332 \\
\hline Current expenditure & 39,097 & 45,050 & 48,088 & 61,360 \\
\hline Total expenditure and net lending & 57,930 & 65,334 & 76,040 & 74,860 \\
\hline Salaries & 17,357 & 18,103 & 21,457 & 24,078 \\
\hline Total spending on goods and services & 10,402 & 12,596 & 13,017 & 18,300 \\
\hline
\end{tabular}

Sources: Burundi authorities; and Fund staff estimates.

1/ Maintenance of vehicles and infrastructure. 
Table 21. Burundi: Functional Structure of Government Operating and Capital Expenditure,1995-98 1/

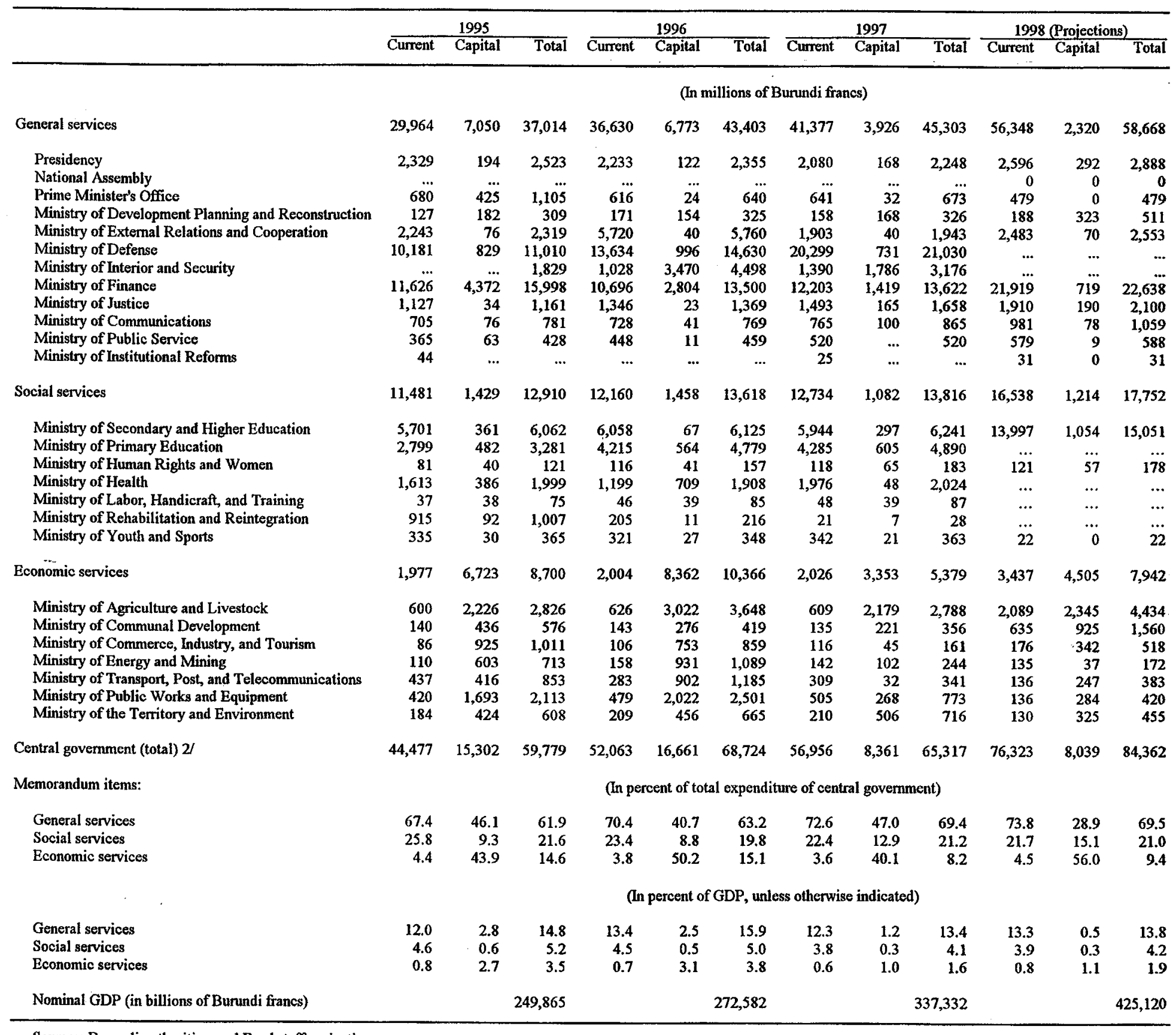

Sources: Burundi authorities; and Fund staff projections.

1/ Excludes debt service.

2/Because of classification problems, the exclusion of some government agencies, and the incomplete imputation of all expenditure by ministry, the totals in this line do not correspond to totals in Table 18. 
Table 22. Burundi: Structure of Central Government Employment and Salaries, 1996

\begin{tabular}{|c|c|c|c|c|c|c|}
\hline & \multicolumn{3}{|c|}{ Number Employed 1/ } & \multicolumn{3}{|c|}{ Annual Salaries } \\
\hline & \multirow{2}{*}{\multicolumn{2}{|c|}{ Statutory Contractual }} & \multirow[t]{2}{*}{ Total } & Statutory & ntractual & Total \\
\hline & & & & \multicolumn{3}{|c|}{ (In millions of Burundi francs) } \\
\hline General services & 2,512 & 2,207 & 4,719 & 2,239 & 1,955 & 12,387 \\
\hline Presidency & 76 & 319 & 395 & 56 & 468 & 524 \\
\hline National Ássembly & 22 & 132 & 154 & 36 & 180 & 216 \\
\hline Prime Minister's Office & 6 & 27 & 33 & 3 & 11 & 14 \\
\hline Ministry of Development Planning and Reconstruction & 52 & 8 & 60 & 44 & 9 & 53 \\
\hline Ministry of External Relations and Cooperation & 108 & 43 & 151 & 641 & 253 & 894 \\
\hline Ministry of Defense & $\ldots$ & $\ldots$ & $\ldots$ & $\ldots$ & $\ldots$ & 8,193 \\
\hline Ministry of Interior and Security & 724 & 552 & 1,276 & 322 & 586 & 908 \\
\hline Ministry of Finance & 387 & 259 & 646 & 438 & 110 & 548 \\
\hline Ministry of Justice & 1,009 & 809 & 1,818 & 622 & 322 & 944 \\
\hline Ministry of Communications & 7 & 8 & 15 & 5 & 2 & 8 \\
\hline Ministry of Public Service & 111 & 37 & 148 & 66 & 11 & 77 \\
\hline Ministry of Institutional Reforms & 10 & 13 & 23 & 6 & 2 & 8 \\
\hline Social services & 17,083 & 4,061 & 21,144 & 7,186 & 876 & 8,062 \\
\hline Ministry of Secondary and Higher Education & 4,337 & 672 & 5,009 & 2,325 & 115 & 2,440 \\
\hline Ministry of Primary Education & 10,788 & 984 & 11,772 & 3,861 & 217 & 4,078 \\
\hline Ministry of Human Rights and Women & 69 & 75 & 144 & 33 & 13 & 46 \\
\hline Ministry of Health & 1,592 & 1,721 & 3,313 & 831 & 422 & 1,253 \\
\hline Ministry of Labor, Handicraft, and Training & 62 & 33 & 95 & 32 & 6 & 37 \\
\hline Ministry of Rehabilitation and Reintegration & 12 & 15 & 27 & 8 & 3 & 11 \\
\hline Ministry of Youth and Sports & 223 & 561 & 784 & 96 & 101 & 197 \\
\hline Economic services & 1,022 & 2,133 & 3,155 & 571 & 436 & 1,007 \\
\hline Ministry of Agriculture and Livestock & 409 & 1,090 & 1,499 & 211 & 232 & 443 \\
\hline Ministry of Communal Development & 83 & 30 & 113 & 42 & 13 & 55 \\
\hline Ministry of Commerce, Industry, and Tourism & 89 & 38 & 127 & 52 & 8 & 60 \\
\hline Ministry of Energy and Mining & 97 & 104 & 201 & 78 & 26 & 104 \\
\hline Ministry of Transport, Post, and Telecommunications & 25 & 231 & 256 & 21 & 53 & 75 \\
\hline Ministry of Public Works and Equipment & 175 & 535 & 710 & 102 & 90 & 192 \\
\hline Ministry of the Territory and Environment & 144 & 105 & 249 & 65 & 14 & 79 \\
\hline Central government (total) & 20,617 & 8,401 & 29,018 & 9,996 & 3,267 & 21,456 \\
\hline Civil administration & 20,617 & 8,401 & 29,018 & 9,996 & 3,267 & 13,263 \\
\hline Military administration & $\ldots$ & $\ldots$ & $\ldots$ & $\ldots$ & $\ldots$ & 8,193 \\
\hline
\end{tabular}

Source: Ministry of Finance.

1/ As of end-December 1996. 
Table 23. Burundi: Size, Composition, and Gross Salaries of the Civil Service, 1983-96

\begin{tabular}{|c|c|c|c|c|c|c|c|c|c|c|c|c|c|c|}
\hline & & & & \multicolumn{10}{|c|}{$\begin{array}{c}\text { Composition and Gross Salaries 2/ 3/ } \\
\text { Statutory }\end{array}$} & \multirow{3}{*}{$\begin{array}{r}\text { Difference } \\
\text { in Total } \\
\text { Size 3/ } \\
\end{array}$} \\
\hline & \multicolumn{3}{|c|}{ Size of Civil Service 1/ } & \multicolumn{2}{|c|}{ Teachers } & \multicolumn{2}{|c|}{ Nonteachers } & \multicolumn{2}{|c|}{ (Teachers \& nonteachers) } & \multicolumn{2}{|c|}{ Contractual } & \multicolumn{2}{|c|}{ Total civil service } & \\
\hline & Statutory & Contractual & Total 3/ & Number & Salaries & Number & Salaries & Number & Salaries & Number & Salaries & Size 3/ & Salaries & \\
\hline 1983 & 11,386 & 9,991 & 21,377 & 7,979 & 1,679 & 5,224 & 1,197 & 13,203 & 2,876 & 10,281 & 979 & 23,484 & 3,855 & 2,107 \\
\hline 1984 & 12,152 & 8,567 & 20,719 & 8,679 & 1,870 & 5,382 & 1,328 & 14,061 & 3,198 & 8,666 & 855 & 22,727 & 4,053 & 2,008 \\
\hline 1985 & 13,345 & 8,073 & 21,418 & 9,551 & 2,125 & 5,808 & 1,482 & 15,359 & 3,606 & 8,243 & 836 & 23,602 & 4,443 & 2,184 \\
\hline 1986 & 12,885 & 8,102 & 20,987 & 9,017 & 2,441 & 6,139 & 1,660 & 15,156 & 4,101 & 8,243 & 892 & 23,399 & 4,992 & 2,412 \\
\hline 1987 & 13,643 & 8,398 & 22,041 & 9,664 & 2,607 & 6,365 & 1,729 & 16,029 & 4,337 & 8,556 & 918 & 24,585 & 5,254 & 2,544 \\
\hline 1988 & 16,995 & 8,480 & 25,475 & 10,187 & 3,077 & 6,757 & 2,005 & 16,944 & 5,083 & 8,703 & 971 & 25,647 & 6,054 & 172 \\
\hline 1989 & 17,364 & 8,768 & 26,132 & 11,814 & 3,511 & 6,986 & 2,224 & 18,800 & 5,736 & 9,012 & 1,100 & 27,812 & 6,836 & 1,680 \\
\hline 1990 & 18,535 & 8,941 & 27,476 & 12,883 & 4,204 & 7,673 & 2,611 & 20,556 & 6,814 & 9,230 & 1,211 & 29,786 & 8,026 & 2,310 \\
\hline 1991 & 19,694 & 8,640 & 28,334 & 13,322 & 4,468 & 7,877 & 2,705 & 21,199 & 7,172 & 8,640 & 1,173 & 29,839 & 8,346 & 1,505 \\
\hline 1992 & 19,823 & 8,501 & 28,324 & 13,896 & 5,144 & 7,779 & 2,872 & 21,675 & 8,016 & 8,501 & 1,207 & 30,176 & 9,223 & 1,852 \\
\hline 1993 & 20,439 & 8,168 & 28,607 & 14,540 & 6,059 & 8,145 & 3,186 & 22,685 & 9,245 & 8,361 & 1,271 & 31,046 & 10,516 & 2,439 \\
\hline 1994 & 20,058 & 8,326 & 28,384 & 14,267 & 6,393 & 8,345 & 3,271 & 22,612 & 9,663 & 8,326 & 1,317 & 30,938 & 10,981 & 2,554 \\
\hline 1995 & 20,159 & 8,676 & 28,835 & 14,666 & 6,265 & 8,264 & 3,165 & 22,930 & 9,430 & 8,676 & 1,484 & 31,606 & 10,914 & 2,771 \\
\hline 1996 & 20,617 & 8,401 & 29,018 & 15,125 & 6,484 & 8,508 & 3,330 & 23,633 & 9,814 & 8,158 & 1,505 & 31,791 & 11,320 & 2,773 \\
\hline
\end{tabular}

Sources: Ministry of Finance; and Ministry of Public Service.

1/ The source for these series is the Ministry of Finance.

2/ The source for these series is the Ministry of Public Services; gross salaries are in millions of Burundi francs.

3/ The large differences between the two sources' totals for the size of the civil service, as well as for the number of statutory and contractual workers, remain to be explained; they demonstrate the need for more accurate information and a better management of the civil service and the government payroll. 
Table 24. Burundi: Flows of On-Lending to, and Repayments by, Public Enterprises, 1994-98 1/

(In millions of Burundi francs)

\begin{tabular}{lrrrrr}
\hline & 1994 & 1995 & 1996 & $\begin{array}{r}1997 \\
\text { Est. }\end{array}$ & $\begin{array}{r}1998 \\
\text { Proj. }\end{array}$ \\
\hline On-lending to public enterprises & 1,133 & 1,258 & 0 & 0 & 0 \\
& & & & & \\
REGIDESO & 402 & 732 & 0 & 0 & 0 \\
ONATEL & 123 & 130 & 0 & 0 & 0 \\
OCIBU & 425 & 331 & 0 & 0 & 0 \\
OTB & 31 & 16 & 0 & 0 & 0 \\
COGERCO & $\ldots$ & 49 & 0 & 0 & 0 \\
Other enterprises & 152 & $\ldots$ & 0 & 0 & 0 \\
& & & & & \\
Repayments by public enterprises & 3,259 & 1,789 & 517 & 3,390 & 0 \\
REGIDESO & & & & & \\
ONATEL & 861 & 20 & $\ldots$ & 724 & 0 \\
OCIBU & 734 & 311 & 286 & 361 & 0 \\
OTB & 734 & 149 & 231 & 804 & 0 \\
COGERCO & 93 & $\ldots$ & $\ldots$ & 0 & 0 \\
SOSUMO & 56 & 44 & 0 & 53 & 0 \\
NOVOTEL & 704 & 351 & 0 & 429 & 0 \\
BCC & 64 & 20 & $\ldots$ & 0 & 0 \\
Other enterprises & 13 & 16 & $\ldots$ & 0 & 0 \\
\hline
\end{tabular}

Sources: Ministry of Finance; and Fund staff estimates and projections.

1/ For full names of public enterprises, see Table 27. 
Table 25. Burundi: Outstanding Arrears on External Debt, 1995-98

(In millions of U.S. dollars)

\begin{tabular}{|c|c|c|c|c|c|c|c|c|c|c|c|c|}
\hline & \multicolumn{3}{|c|}{1995} & \multicolumn{3}{|c|}{1996} & \multicolumn{3}{|c|}{1997} & \multicolumn{3}{|c|}{1998 (Pröjections) } \\
\hline & Principal & Interest & Total & Principal & Interest & Total & Principal & Interest & $\overline{\text { Total }}$ & Principal & Interest & Total \\
\hline Multilateral debt & 4.9 & 3.6 & 8.5 & 10.9 & 5.2 & 16.1 & 11.6 & 2.9 & 14.5 & 16.3 & 3.6 & 19.9 \\
\hline IDA & 0.2 & 0.1 & 0.3 & 0.4 & 0.3 & 0.7 & 0.0 & 0.0 & 0.0 & 0.0 & 0.0 & 0.0 \\
\hline African Development Bank & 2.4 & 1.6 & 4.0 & 1.8 & 1.0 & 2.8 & 0.0 & 0.0 & 0.0 & 0.0 & 0.0 & 0.0 \\
\hline African Development Fund & 1.1 & 1.5 & 2.6 & 1.6 & 1.9 & 3.5 & 0.0 & 0.0 & 0.0 & 0.0 & 0.0 & 0.0 \\
\hline European Union & 0.1 & 0.1 & 0.1 & 0.8 & 0.4 & 1.3 & 1.5 & 0.6 & 2.1 & 2.1 & 0.9 & 3.0 \\
\hline European Investment Bank & 0.0 & 0.1 & 0.1 & 0.0 & 0.2 & 0.2 & 0.3 & 0.6 & 0.9 & 0.5 & 0.7 & 1.2 \\
\hline \multicolumn{13}{|l|}{ Organization of Petroleum } \\
\hline Exporting Countries & 0.0 & 0.0 & 0.0 & 2.0 & 0.2 & 2.2 & 3.7 & 0.4 & 4.1 & 5.4 & 0.5 & 5.9 \\
\hline Arab Bank for Economic Development & & & & & & & & & & & & \\
\hline in Africa & 1.0 & 0.3 & 1.3 & 3.4 & 0.8 & 4.3 & 5.7 & 1.2 & 6.9 & 7.8 & 1.4 & 9.2 \\
\hline \multicolumn{13}{|l|}{ International Fund for Agricultural } \\
\hline Development & 0.0 & 0.0 & 0.0 & 0.7 & 0.2 & 0.9 & 0.0 & 0.0 & 0.0 & 0.0 & 0.0 & 0.0 \\
\hline Arab League & 0.1 & 0.0 & 0.1 & 0.2 & 0.0 & 0.2 & 0.3 & 0.0 & 0.3 & 0.3 & 0.0 & 0.3 \\
\hline United Nations Development Program & 0.0 & 0.0 & 0.0 & 0.1 & 0.1 & 0.1 & 0.1 & 0.1 & 0.2 & 0.2 & 0.1 & 0.3 \\
\hline Bilateral debt & 3.7 & 1.3 & 5.0 & 13.4 & 4.3 & 17.7 & 21.1 & 6.9 & 28.0 & 27.0 & 9.0 & 36.0 \\
\hline \multicolumn{13}{|l|}{ France (Caisse Française de } \\
\hline Développement) & 0.8 & 0.0 & 0.8 & 2.6 & 1.7 & 4.3 & 4.3 & 3.2 & 7.5 & 5.9 & 4.3 & 10.2 \\
\hline \multicolumn{13}{|l|}{ Abu Dhabi Fund for Arab } \\
\hline Economic Development & 0.0 & 0.0 & 0.0 & 0.5 & 0.1 & 0.6 & 0.9 & 0.1 & 1.0 & 1.3 & 0.1 & 1.4 \\
\hline China & 2.3 & 0.0 & 2.3 & 6.1 & 0.0 & 6.1 & 8.5 & 0.0 & 8.5 & 10.3 & 0.0 & 10.3 \\
\hline Saudi Arabia & 0.0 & 0.0 & 0.0 & 1.6 & 0.4 & 2.0 & 2.7 & 0.8 & 3.5 & 4.1 & 1.1 & 5.2 \\
\hline Kuwait & 0.6 & 0.2 & 0.8 & 2.6 & 0.7 & 3.3 & 4.4 & 1.1 & 5.5 & $\begin{array}{l}7.1 \\
5.1\end{array}$ & 1.4 & 6.5 \\
\hline Japan & 0.0 & 1.1 & 1.1 & 0.0 & 1.4 & 1.4 & 0.3 & 1.8 & 2.1 & 0.3 & 2.1 & 2.4 \\
\hline Other debt $1 /$ & 0.0 & 0.0 & 0.0 & 2.8 & 1.6 & 4.4 & 6.8 & 4.6 & 11.4 & 8.0 & 6.0 & 14.0 \\
\hline Total stock of arrears & 8.6 & 4.9 & 13.5 & 27.2 & 11.0 & 38.2 & 39.5 & 14.4 & 53.9 & 51.3 & 18.6 & 70.0 \\
\hline
\end{tabular}

Sources: Ministry of Finance; and Fund staff estimates and projections.

1/ This item is introduced to account for discrepancies between overall stock of external arrears and its components by creditor. 
Table 26. Burundi: Domestic Public Debt by Creditor and by Instrument, 1994-97 (End of period; in billions of Burundi francs)

\begin{tabular}{|c|c|c|c|c|}
\hline & 1994 & 1995 & 1996 & 1997 \\
\hline Domestic public debt by creditor $1 /$ & 14.7 & 19.4 & 27.2 & 39.9 \\
\hline Banking sector & 13.7 & 15.5 & 22.2 & 33.3 \\
\hline Central Bank & 8.6 & 10.5 & 12.3 & 20.6 \\
\hline Ordinary advances & 0.4 & 0.0 & 1.3 & 5.8 \\
\hline Special advances & 7.6 & 6.9 & 6.1 & 5.3 \\
\hline Exceptional advances & 0.2 & 0.0 & 0.0 & 0.0 \\
\hline Particular advances & 0.0 & 2.1 & 4.9 & 8.1 \\
\hline Advances (AfDB capital) & 0.0 & 0.0 & 0.0 & 0.0 \\
\hline Treasury certificates & 0.4 & 1.5 & 0.0 & 1.4 \\
\hline Commercial banks & 3.7 & 3.3 & 8.6 & 11.2 \\
\hline Investment bonds & 0.0 & 0.0 & 0.0 & 0.0 \\
\hline Treasury certificates & 2.5 & 1.7 & 8.6 & 11.2 \\
\hline Consortium advances (SOSUMO) & 0.0 & 0.0 & 0.0 & 0.0 \\
\hline Other & 1.2 & 1.6 & $\cdots$ & ... \\
\hline CAMOFI & 0.0 & 0.0 & 0.0 & 0.0 \\
\hline Advances & 0.0 & 0.0 & 0.0 & 0.0 \\
\hline Treasury bonds & 0.0 & 0.0 & 0.0 & 0.0 \\
\hline Investment bonds & 0.0 & 0.0 & 0.0 & 0.0 \\
\hline Treasury certificates & 0.0 & 0.0 & 0.0 & 0.0 \\
\hline Consortium advances (SOSUMO) & 0.0 & 0.0 & 0.0 & 0.0 \\
\hline $\mathrm{CCP}$ & 1.4 & 1.7 & 1.3 & 1.5 \\
\hline Other financial establishments & 0.1 & 0.1 & 0.1 & 0.5 \\
\hline Treasury bonds & 0.1 & 0.1 & 0.1 & 0.0 \\
\hline Treasury certificates & 0.0 & 0.0 & 0.0 & 0.5 \\
\hline Consortium advances (SOSUMO) & 0.0 & 0.0 & 0.0 & 0.0 \\
\hline Other loans & 0.0 & 0.0 & 0.0 & 0.0 \\
\hline Nonfinancial sector & 0.9 & 3.8 & 4.9 & 6.1 \\
\hline Treasury bonds & 0.0 & 0.0 & 0.0 & 0.0 \\
\hline Treasury certificates & 0.3 & 0.8 & 2.0 & 3.4 \\
\hline Other loans & 0.6 & 3.0 & 2.9 & 2.7 \\
\hline Domestic public debt by instrument & 14.7 & 19.3 & 27.2 & 40.0 \\
\hline Treasury bonds & 0.1 & 0.0 & 0.0 & 0.0 \\
\hline Investment bonds & 0.0 & 0.0 & 0.0 & 0.0 \\
\hline Treasury certificates & 3.2 & 3.9 & 10.6 & 16.5 \\
\hline Advances of the central bank & 8.2 & 9.1 & 12.4 & 19.3 \\
\hline Other loans & 3.2 & 6.3 & 4.2 & 4.2 \\
\hline
\end{tabular}

Source: Bank of the Republic of Burundi.

1/ AfDB is the African Development Bank; CCP is Centre des Chèques Postaux; CAMOFI is Caisse de Mobilisation et de Financement; and SOSUMO is Société Sucrière de Moso. 
Table 27. Burundi: List of Public Enterprises, 1997 1/

\begin{tabular}{ll}
\hline Acronym & \\
\hline Fully owned public enterprises & \\
& \\
AIR BURUNDI & Air Burundi \\
BCC & Burundi Coffee Company \\
CAMOFI & Caisse de Mobilisation et de Financement \\
COGERCO & Compagnie de Gérance Cotonnière \\
COTEBU & Complexe Textile de Bujumbura \\
ECOSAT & Encadrement des Constructions Sociales et Aménagement des Terrains \\
FDC & Fonds de Développement Communal \\
FOSIP & Fonds de Soutien à l'Investissement Privé \\
INABU & Imprimerie Nationale du Burundi \\
ONAPHA & Office National Pharmaceutique \\
ONATEL & Office National des Télécommunications \\
ONATOUR & Office National de la Tourbe \\
OPHAVET & Office Pharmaceutique Vétérinaire \\
OTB & Office du Thé du Burundi \\
OTRACO & Office des Transports en Commun \\
REGIDESO & Régie de Distribution d'Eau et d'Eléctricité \\
SETEMU & Services Techniques et Municipaux \\
SIP & Société Immobilière Publique \\
SOFIDHAR & Société de Fínancement et Développement de l'Habitat Urbain \\
SRDI & Société Régionale de Développement de l'IMBO \\
SRDR & Société Régionale de Développement de Rumonge \\
&
\end{tabular}

Mixed ownership 2/

\begin{tabular}{ll} 
ALCOVIT & Aliments Composés Vitaminés \\
APEE & Agences de Promotion des Echanges Extérieurs \\
BANCOBU & Banque Commerciale du Burundi \\
BBCI & Banque Burundaise pour le Commerce et l'Investissement \\
BCB & Banque de Crédit de Bujumbura \\
BNDE & Banque Nationale pour le Développement Economique \\
BPB & Banque Populaire du Burundi \\
BRARUDI & Brasseries et Limonaderies du Burundi \\
BUMINCO & Burundi Mining Company \\
EPB & Exploitation du Port de Bujumbura \\
FNG & Fonds National de Garantie \\
FPHU & Fonds de Promotion de l'Habitat Urbain \\
HOT. SOURCE DU NIL & Hotel Source du Nil \\
HPB & Huilerie de Palme du Burundi \\
NOVOTEL & Hotel Novotel \\
OCIBU & Office des Cultures Industrielles du Burundi \\
SBF & Société Burundaise de Financement \\
SER & Société d'Exploitation de la Ferme Randa \\
SOBUGEA & Société Burundaise de Gestion Portuaire \\
SOCABU & Société d'Assurances du Burundi \\
SODECO & Société de Déparchage et de Commercialisation \\
SOGEMAC & Société de Gestion du Marché Central de Bujumbura \\
SOGESTAL KAYANZA & Société de Gestion des Stations de Lavage de Kayanza \\
SOGESTAL KIRIMIRO & Société de Gestion des Stations de Lavage de Kirimiro \\
SOGESTAL KIRUNDO & Société de Gestion des Stations de Lavage de Kirundo \\
SOGESTAL MUMIRWA & Société de Gestion des Stations de Lavage de Mumirwa \\
SOGESTAL NGOZI & Société de Gestion des Stations de Lavage de Ngozi \\
SOKINABU & Société d'Exploitation de Quinquina du Burundi \\
SOSUMO & Société Sucrière de Moso \\
TELECEL BURUNDI & Téléphonie Cellulaire du Burundi \\
UCAR & Union Commerciale d'Assurances et de Réassurances \\
UPC & Usine de Produits en Coton \\
VERRUNDI & Verrerie du Burundi \\
\hline & \\
\hline &
\end{tabular}

Source: Service Chargé des Entreprises Publiques (SCEP).

1/ Following the definition under the new public enterprise code promulgated on March 6, 1996 (loi no 1/002); situation as of December 1997.

2/ For government shares, see Table 28. 
- 33 -

Table 28. Main Economic Indicators of Public Enterprises, 1996

(In millions of Burundi francs, unless otherwise indicated)

\begin{tabular}{|c|c|c|c|c|c|c|c|c|}
\hline & Public Enterprises & $\begin{array}{l}\text { Equity } \\
\text { Capital }\end{array}$ & $\begin{array}{r}\text { Equity } \\
\text { Participation } \\
\text { (In percent) } 1 /\end{array}$ & $\begin{array}{r}\text { Total } \\
\text { Salaries } \\
\text { and Wages }\end{array}$ & $\begin{array}{r}\text { Total } \\
\text { Assets }\end{array}$ & $\begin{array}{l}\text { Total } \\
\text { Debt }\end{array}$ & $\begin{array}{l}\text { Value } \\
\text { Added }\end{array}$ & $\begin{array}{r}\text { Earnings } \\
\text { before } \\
\text { Subsidies }\end{array}$ \\
\hline \multicolumn{9}{|c|}{ Majority state participation } \\
\hline 1 & AIR BURUNDI & 293 & 100 & 172 & 2,166 & 1,529 & 188 & -233 \\
\hline 2 & ALCOVIT & 219 & 90 & 16 & 147 & 42 & 13 & 21 \\
\hline 3 & APEE & 144 & 52 & 21 & 155 & 7 & 10 & .20 \\
\hline 4 & $\mathrm{BBCI}$ & 330 & 79 & 99 & 3,580 & 3,151 & 215 & 14 \\
\hline 5 & $\mathrm{BCC}$ & 25 & 100 & 34 & 653 & 585 & 1 & -51 \\
\hline 6 & BPB & 285 & 86 & 96 & 2,634 & 1,985 & 246 & 17 \\
\hline 7 & CAMOFI & 200 & 100 & 87 & 6,161 & 4,058 & 264 & -560 \\
\hline 8 & COGERCO & 89 & 100 & 159 & 1,853 & 1,480 & 252 & -33 \\
\hline 9 & COTEBU & 2,500 & 100 & 638 & 5,355 & 4,792 & 374 & -580 \\
\hline 10 & ECOSAT & 10 & 100 & 31 & 978 & 789 & -3 & -39 \\
\hline 11 & FDC & 250 & 100 & 12 & 561 & 35 & 32 & 3 \\
\hline 12 & FNG & 326 & 87 & 27 & 189 & 90 & -21 & -58 \\
\hline 13 & FOSIP & 409 & 100 & $\ldots$ & $\ldots$ & $\ldots$ & $\ldots$ & $\ldots$ \\
\hline 14 & FPHU & 409 & 83 & 52 & 3,461 & 1,829 & $\dddot{50}$ & 23 \\
\hline 15 & HOT. SOURCE DU NIL & 761 & 65 & 87 & 1,020 & 746 & 136 & -22 \\
\hline 16 & INABU & 62 & 100 & 60 & 135 & 204 & 11 & -54 \\
\hline 17 & NOVOTEL & 498 & 50 & 161 & 1,313 & 889 & 206 & -98 \\
\hline 18 & OCIBU PATRIMOINE & 77 & 78 & 6 & 14,959 & 12,855 & 261 & $-4,588$ \\
\hline 19 & ONAPHA & 105 & 100 & 53 & 274 & 57 & 114 & 25 \\
\hline 20 & ONATEL & 597 & 100 & 565 & 10,834 & 7,295 & 3,326 & 191 \\
\hline 21 & ONATOUR & 54 & 100 & 33 & 472 & 60 & 86 & 13 \\
\hline 22 & OPHAVET & 10 & 100 & 5 & 43 & 1 & 1 & .6 \\
\hline 23 & OTB & 2,426 & 100 & 670 & 8,025 & 6,353 & 982 & .688 \\
\hline 24 & OTRACO & 427 & 100 & ... & $\ldots$ & $\ldots$ & $\ldots$ & $\ldots$ \\
\hline 25 & REGIDESO & 1,442 & 100 & 995 & 33,436 & 18,977 & 9,012 & $-1,460$ \\
\hline 26 & SBF & 860 & 67 & 85 & 6,488 & 3,689 & 715 & 222 \\
\hline 27 & SETEMU & 178 & 100 & $\ldots$ & $\ldots$ & $\ldots$ & $\ldots$ & $\ldots$ \\
\hline 28 & SIP & 400 & 100 & 57 & 2,874 & 2,272 & 33 & -86 \\
\hline 39 & SOBUGEA & 46 & 90 & 135 & 389 & 245 & 158 & -83 \\
\hline 30 & SODECO & 276 & 82 & 192 & 3,921 & 3,434 & 415 & .188 \\
\hline 31 & SOFIDHAR & 300 & 100 & $\ldots$ & & $\ldots$ & $\ldots$ & $\ldots$ \\
\hline 32 & SOGESTAL KIRIMIRO & 50 & 52 & 140 & 1,552 & 1,434 & 190 & 6 \\
\hline 33 & SOGESTAL MUMIRWA & 30 & 82 & 96 & 1,194 & 1,109 & 75 & -135 \\
\hline 34 & SOSUMO & 2,145 & 99 & 567 & 9,630 & 2,806 & 2,218 & 437 \\
\hline 35 & SRDI & 35 & 100 & 94 & 4,502 & 1,037 & 326 & 2 \\
\hline 36 & SRDR & $\ldots$ & 100 & $\ldots$ & & $\ldots$ & $\ldots$ & $\ldots$ \\
\hline \multirow[t]{2}{*}{37} & VERRUNDI & 1,832 & 66 & 151 & 1,285 & 936 & -75 & -458 \\
\hline & Subtotal & 18,099 & & 5,596 & 130,239 & 84,771 & 19,811 & $-8,466$ \\
\hline \multicolumn{9}{|c|}{ Minority state participation } \\
\hline 38 & BANCOBU & 330 & 14 & 546 & 17,356 & 12,390 & 1,595 & 103 \\
\hline 39 & $\mathrm{BCB}$ & 400 & 17 & 546 & 20,349 & 17,640 & 1,440 & 388 \\
\hline 40 & BNDE & 740 & 43 & 139 & 11,155 & 7,743 & 662 & 2 \\
\hline 41 & BRARUDI & 506 & 41 & 1,776 & 15,448 & 11,271 & 3,596 & 259 \\
\hline 42 & BUMINCO & 498 & 48 & 15 & 36 & 82 & -5 & -37 \\
\hline 43 & EPB & 350 & 43 & 85 & 583 & 244 & 70 & -39 \\
\hline 44 & HPB & 117 & 49 & 13 & 90 & 419 & 3 & -175 \\
\hline 45 & OCIBU SOCIAL & 77 & 35 & 181 & 1,563 & 160 & $\ldots$ & -486 \\
\hline 46 & SER & 162 & 35 & 8 & 117 & 214 & 12 & -22 \\
\hline 47 & SOCABU & 180 & 39 & 299 & 5,232 & 368 & 651 & 93 \\
\hline 48 & SOGEMAC & 20 & 40 & 23 & 136 & 65 & 127 & 31 \\
\hline 49 & SOGESTAL KAYANZA & 30 & 14 & 115 & 2,784 & 2,604 & 524 & 91 \\
\hline 50 & SOGESTAL KIRUNDO & 31 & 48 & $\ldots$ & $\ldots$ & $\ldots$ & $\ldots$ & \\
\hline 51 & SOGESTAL NGOZI & 51 & 27 & 142 & 2,468 & $2,46 \dddot{2}$ & 325 & 64 \\
\hline 52 & SOKINABU & 105 & 17 & 6 & 114 & 8 & 5 & -8 \\
\hline 53 & TELECEL BURUNDI & 562 & 40 & 55 & 759 & 287 & 225 & -13 \\
\hline 54 & UCAR & 150 & 19 & 74 & 975 & 304 & 136 & -12 \\
\hline \multirow[t]{3}{*}{55} & UPC & 105 & 19 & 21 & 418 & 412 & 104 & 18 \\
\hline & Subtotal & 4,413 & & 4,044 & 79,583 & 56,673 & 9,470 & 257 \\
\hline & Total & 22,512 & & 9,640 & 209,822 & 141,444 & 29,281 & $-8,209$ \\
\hline
\end{tabular}

Source: Service Charge des Entreprises Publiques (SCEP).

1/ Direct or indirect equity participation of government in public enterprises. 
Table 29. Burundi: Monetary Survey, 1994-98

\begin{tabular}{|c|c|c|c|c|c|c|}
\hline & 1994 & 1995 & 1996 & 1997 & \multicolumn{2}{|c|}{1998} \\
\hline & \multicolumn{4}{|c|}{ December } & \multirow[t]{2}{*}{ June } & \multirow{2}{*}{$\begin{array}{l}\text { Dec. } \\
\text { Proj. }\end{array}$} \\
\hline & \multicolumn{4}{|c|}{ (In billions of Burundi francs) } & & \\
\hline Net foreign assets & 37.3 & 45.2 & 37.1 & 35.6 & 26.2 & 36.3 \\
\hline Central bank & 35.7 & 43.5 & 31.6 & 33.7 & 26.9 & 37.0 \\
\hline Deposit money banks & 1.6 & 1.7 & 5.5 & 1.9 & .0 .7 & -0.7 \\
\hline Net domestic assets & 15.0 & 4.8 & 20.1 & 27.6 & 37.9 & 34.5 \\
\hline Domestic credit & 42.1 & 35.5 & 54.7 & 68.0 & 84.2 & 84.8 \\
\hline Net claims on the government & 1.0 & 1.0 & 11.7 & 23.4 & 30.4 & 27.2 \\
\hline Central government & 8.6 & 5.7 & 16.7 & 27.7 & 34.5 & 31.3 \\
\hline Treasury & 12.0 & 12.1 & 20.9 & 31.4 & 38.2 & 35.0 \\
\hline Other central government & -3.4 & -6.4 & -4.1 & .3 .7 & -3.7 & $\mathbf{- 3 . 7}$ \\
\hline Other government & -7.7 & -4.6 & -5.0 & $-4,3$ & -4.1 & -4.1 \\
\hline Credit to the economy & 41.1 & 34.5 & 43.0 & 44.7 & 53.8 & 57.6 \\
\hline Claims on public enterprises & 2.7 & 1.3 & 1.7 & 2.2 & 2.2 & 2.2 \\
\hline Claims on private sector & 38.4 & 33.2 & 41.3 & 42.4 & 51.6 & 55.5 \\
\hline Other items (net) & -27.0 & -30.7 & -34.6 & -40.5 & -46.3 & -50.3 \\
\hline Money and quasi money & 52.3 & 50.0 & 57.2 & 63.1 & 64.1 & 70.9 \\
\hline Money & 39.6 & 40.0 & 43.4 & 48.4 & 49.5 & 54.7 \\
\hline \multirow[t]{2}{*}{ Quasi money } & 12.7 & 9.9 & 13.8 & 14.7 & 14.6 & 16.1 \\
\hline & \multicolumn{6}{|c|}{ (Annual change in percent of the beginning-of-period broad money supply) } \\
\hline Net foreign assets & 28.9 & 15.1 & -16.2 & -2.6 & -24.1 & 1.2 \\
\hline Central bank & 26.2 & 14.8 & -23.7 & 3.6 & -15.0 & 5.3 \\
\hline Deposit money banks & 2.7 & 0.3 & 7.5 & -6.3 & -9.1 & -4.1 \\
\hline Net domestic assets & 6.1 & -19.6 & 30.6 & 13.0 & 22.4 & 11.0 \\
\hline Domestic credit & 17.3 & -12.5 & 38.3 & 23.4 & 41.9 & 26.6 \\
\hline Net claims on the government & 9.0 & 0.1 & 21.4 & 20.4 & 21.0 & 6.0 \\
\hline Credit to the economy & 8.3 & -12.7 & 16.9 & 3.0 & 21.0 & 20.6 \\
\hline Claims on public enterprises & 3.0 & -2.7 & 0.7 & 0.9 & -0.7 & -0.1 \\
\hline Claims on private sector & 5.3 & -9.9 & 16.2 & 2.0 & 21.7 & 20.6 \\
\hline Money and quasi money & 35.0 & -4.5 & 14.4 & 10.4 & -1.8 & 12.2 \\
\hline Memorandum item: & & & & & & \\
\hline Velocity (GDP/M2) & 4.5 & 5.0 & 4.8 & 5.3 & $\cdots$ & 6.0 \\
\hline
\end{tabular}

Sources: Burundi authorities; and Fund staff projections. 
Table 30. Burundi: Summary Accounts of the Bank of the Republic of Burundi, 1994-98 (In millions of Burundi francs; end of period)

\begin{tabular}{|c|c|c|c|c|c|c|}
\hline & \multirow[t]{2}{*}{1994} & \multirow[t]{2}{*}{1995} & \multirow[t]{2}{*}{1996} & \multirow[t]{2}{*}{.1997} & \multicolumn{2}{|c|}{1998} \\
\hline & & & & & Mar. & June \\
\hline $\begin{array}{l}\text { Foreign assets } \\
\text { Of which }\end{array}$ & $52,812.1$ & $59,836.6$ & $46,630.6$ & $47,918.3$ & $44,060.9$ & $39,355.3$ \\
\hline Reserve position in Fund & $2,106.8$ & $2,422.4$ & $2,711.1$ & $3,232.3$ & $3,264.3$ & $3,348.0$ \\
\hline SDR holdings 1/ & 51.2 & 19.1 & 36.9 & 26.3 & 99.8 & 33.9 \\
\hline Gold holdings & 216.7 & 249.2 & 276.9 & 332.5 & 335.7 & 344.3 \\
\hline Credit to the government & $8,698.2$ & $10,503.6$ & $12,442.5$ & $20,702.2$ & $24,052.7$ & $28,907.2$ \\
\hline Ordinary $2 /$ & 438.1 & 0.0 & $1,332.2$ & $5,870.9$ & $4,149.0$ & $4,981.1$ \\
\hline Special 3/ & $7,615.5$ & $6,873.8$ & $6,106.2$ & $5,344.50$ & $4,778.3$ & $4,609.8$ \\
\hline Exceptional 4/ & 200.0 & $2,160.0$ & $4,920.0$ & $8,070.0$ & $13,655.0$ & $13,200.0$ \\
\hline Particular 5/ & 0.0 & 0.0 & 0.0 & 0.0 & 0.0 & 0.0 \\
\hline African Development Bank subscription & 29.0 & 0.0 & 0.0 & 0.0 & 0.0 & 0.0 \\
\hline Postal deposit account & 0.0 & 0.0 & 0.0 & 0.0 & 0.0 & 0.0 \\
\hline Treasury certificate advances & 415.6 & $1,469.8$ & 84.1 & $1,416.8$ & $1,470.4$ & $6,116.3$ \\
\hline Other advances & 0.0 & 0.0 & 0.0 & 0.0 & 0.0 & 0.0 \\
\hline Credit to banks & $2,528.0$ & $2,145.6$ & $9,239.3$ & $2,387.0$ & $2,970.2$ & $8,694.8$ \\
\hline Credit to other financial institutions & 633.9 & $1,371.8$ & 761.2 & $1,613.0$ & $1,449.2$ & $1,161.8$ \\
\hline Credit to official entities & 25.0 & 25.0 & 25.0 & 25.0 & 25.0 & 25.0 \\
\hline Credit to the private sector & 419.7 & $1,486.1$ & $1,562.5$ & $1,180.6$ & $1,206.7$ & $1,262.4$ \\
\hline Other assets & $3,000.0$ & $2,151.4$ & $2,150.0$ & $3,150.6$ & $3,007.5$ & $2,874.5$ \\
\hline Total assets $=$ Liabilities & $68,116.9$ & $77,520.1$ & $72,811.0$ & $76,976.7$ & $76,772.2$ & $82,281.1$ \\
\hline Reserve money & $22,292.5$ & $22,114.1$ & $26,346.3$ & $26,856.2$ & $26,115.9$ & $28,615.9$ \\
\hline Currency outside banks & $19,839.9$ & $20,132.0$ & $24,762.9$ & $24,692.7$ & $24,069.9$ & $26,879.1$ \\
\hline Deposits by banks & $1,803.2$ & $1,365.7$ & $1,211.7$ & $1,074.9$ & 944.3 & 640.9 \\
\hline Deposits by other financial institutions & 6.3 & 13.0 & 31.5 & 449.7 & 315.8 & 178.0 \\
\hline Deposits by other official entities & 641.9 & 563.1 & 295.9 & 470.1 & 762.0 & 891.7 \\
\hline Other monetary liabilities & 1.2 & 40.3 & 44.3 & 168.8 & 23.9 & 26.2 \\
\hline Government deposits & $7,713.8$ & $10,837.0$ & $7,305.9$ & $6,082.2$ & $7,422.8$ & $6,537.6$ \\
\hline Central government & $6,077.4$ & $8,207.7$ & $5,462.2$ & $4,705.0$ & $5,775.5$ & $5,104.6$ \\
\hline Government agencies & $1,443.5$ & $2,568.3$ & $1,700.6$ & $1,122.9$ & $1,453.6$ & $1,225.4$ \\
\hline Local administration & 192.9 & 61.0 & 143.1 & 254.3 & 193.7 & 207.6 \\
\hline Import deposits & 842.0 & $1,163.5$ & 540.4 & $2,230.3$ & $1,630.9$ & $2,563.3$ \\
\hline $\begin{array}{l}\text { Foreign liabilities } \\
\text { Of which }\end{array}$ & $17,112.1$ & $16,385.5$ & $15,007.8$ & $14,222.3$ & $13,694.7$ & $12,472.1$ \\
\hline Medium- and long-term foreign borrowing 6 / & $14,424.4$ & $14,118.6$ & $13,029.7$ & $12,244.2$ & $11,889.8$ & $10,342.0$ \\
\hline SDR allocation & $4,924.1$ & $5,661.9$ & $6,336.7$ & $7,554.8$ & $7,629.6$ & $7,825.1$ \\
\hline Other liabilities & $15,232.4$ & $21,358.1$ & $17,274.0$ & $20,030.9$ & $20,278.3$ & $24,267.1$ \\
\hline
\end{tabular}

Source: Bank of the Republic of Burundi.

1/ Gold valued at SDR 35 per fine troy ounce.

2/ Ordinary advances are extended to the government to cover ordinary budget operations; their level cannot exceed 10 percent of the previous year's current revenue.

3/ Special advances are extended to finance-selected projects. They have a maturity of ten years. Before 1982, they carried no interest. They are not included in calculating the limit on ordinary advances.

4/ Exceptional advances were introduced in 1982 to consolidate at the end of each year the outstanding amounts of ordinary advances that were not repaid.

5/ Particular advances are short-term loans.

$6 /$ Includes borrowing from the Fund. 
Table 31. Burundi: Summary Accounts of Deposit Money Banks, 1994-98 (In millions of Burundi francs; end of period)

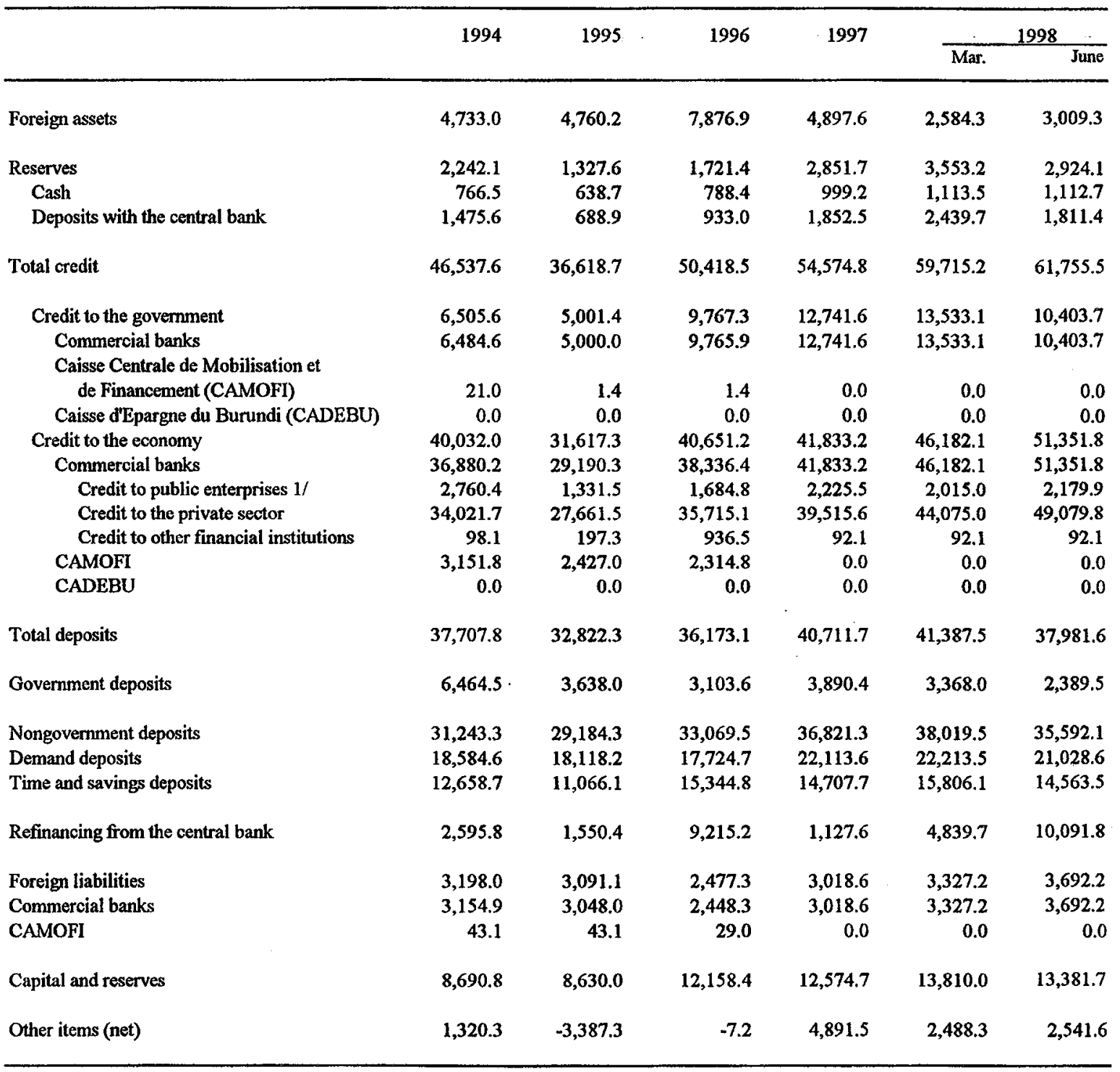

Source: Burundi authorities.

1/ Includes shares in the capital of public enterprises. 
Table 32. Burundi: Summary Accounts of Burundi Development Bank (BNDE), Burundi Financing Company (SBF), Caisse Centrale de Mobilisation et de Financement (CAMOFI), and Société de Gestion Financière (SOGEFI) 1994-98

(In millions of Burundi francs; end of period)

\begin{tabular}{|c|c|c|c|c|c|}
\hline & 1994 & 1995 & 1996 & 1997 & $\begin{array}{l}1998 \\
\text { Mar. }\end{array}$ \\
\hline \multicolumn{6}{|l|}{ BNDE } \\
\hline Credit to the economy & $7,706.5$ & $8,021.4$ & $8,631.1$ & $7,667.3$ & $7,297.3$ \\
\hline Of which: private sector & $6,711.7$ & $7,395.8$ & $8,168.7$ & $7,263.5$ & $5,860.0$ \\
\hline Other assets & $1,237.4$ & $1,787.9$ & $2,329.8$ & $3,030.6$ & $3,459.3$ \\
\hline Assets $=$ Liabilities & $8,943.9$ & $9,809.2$ & $10,960.9$ & $10,697.8$ & $10,755.6$ \\
\hline \multicolumn{6}{|l|}{ Liabilities to other financial } \\
\hline Foreign liabilities & $4,495.9$ & $5,117.0$ & $5,222.0$ & $5,602.5$ & $5,047.3$ \\
\hline Capital and reserves & $2,230.7$ & $2,669.5$ & $3,217.5$ & $3,643.3$ & $3,757.9$ \\
\hline Other liabilities & $2,113.1$ & $1,862.5$ & $1,617.1$ & $1,452.0$ & $1,951.4$ \\
\hline \multicolumn{6}{|l|}{ SBF } \\
\hline Reserves & 23.3 & 34.4 & 53.8 & 245.2 & 82.8 \\
\hline Credit to the economy & $4,266.0$ & $4,715.6$ & $5,116.9$ & $4,577.4$ & $4,551.0$ \\
\hline Of which: private sector & $3,547.5$ & $3,799.1$ & $4,382.6$ & $3,889.5$ & $3,800.4$ \\
\hline Other assets & $1,345.3$ & $1,291.7$ & $1,216.4$ & $1,438.9$ & $1,295.7$ \\
\hline Assets $=$ Liabilities & $5,634.6$ & $6,041.7$ & $6,386.5$ & $6,261.5$ & $5,929.7$ \\
\hline Capital and reserves & $1,025.5$ & 911.8 & $1,139.0$ & $1,494.6$ & $1,297.1$ \\
\hline Other liabilities & $4,609.1$ & $5,129.9$ & $5,247.5$ & $4,765.9$ & $4,632.5$ \\
\hline \multicolumn{6}{|l|}{ CAMOFI 1/ } \\
\hline Foreign assets & $\ldots$ & $\ldots$ & $\ldots$ & 342.1 & 167.9 \\
\hline Credit to government & & & & 25.0 & 202.4 \\
\hline Credit to the economy & $\ldots$ & $\ldots$ & ... & $3,320.7$ & $3,308.6$ \\
\hline Of which: private sector & $\ldots$ & $\ldots$ & $\ldots$ & $2,690.1$ & $2,701.9$ \\
\hline Other assets & $\cdots$ & $\cdots$ & ... & $1,176.0$ & $1,175.3$ \\
\hline Assets $=$ Liabilities & ... & $\cdots$ & $\cdots$ & $4,863.8$ & $4,854.2$ \\
\hline Government deposits & & & & $2,104,4$ & $2,115.6$ \\
\hline Nongovernment deposits & & & & 446.2 & 665.4 \\
\hline Capital and reserves & $\ldots$ & $\ldots$ & $\ldots$ & -239.4 & -251.4 \\
\hline Foreign liabilities & $\ldots$ & $\ldots$ & $\ldots$ & 29.0 & 29.0 \\
\hline Other liabilities & $\cdots$ & $\ldots$ & $\cdots$ & $2,523.6$ & $2,295.7$ \\
\hline \multicolumn{6}{|l|}{ SOGEFI $2 /$} \\
\hline Foreign assets & 12.8 & 15.5 & 15.3 & $\ldots$ & $\ldots$ \\
\hline Credit to the economy & 511.0 & 865.6 & 910.6 & $\ldots$ & ... \\
\hline Of which: private sector & 482.9 & 802.0 & 847.0 & ... & ... \\
\hline Other assets & 422.8 & 145.3 & 176.9 & $\ldots$ & $\ldots$ \\
\hline Assets = Liabilities & 946.6 & $1,026.4$ & $1,102.8$ & $\cdots$ & $\ldots$ \\
\hline Capital and reserves & 243.2 & 370.7 & 485.6 & $\ldots$ & ... \\
\hline Other liabilities & 703.4 & 655.7 & 617.2 & $\cdots$ & ... \\
\hline
\end{tabular}

Source: Burundi authorities.

1/ CAMOFI was reclassified as a nonbank financial institution in early 1997. 2/ SOGEFI was created in 1992. 
Table 33. Burundi: Outstanding Amount of 'Treasury Certificates, 1994-98 1/ (In millions of Burundi francs; period average, unless otherwise specified)

\begin{tabular}{|c|c|c|c|c|c|c|}
\hline & \multirow[t]{2}{*}{1994} & \multirow[t]{2}{*}{1995} & \multirow[t]{2}{*}{1996} & \multirow[t]{2}{*}{1997} & \multicolumn{2}{|c|}{-1998} \\
\hline & & & & & Mar. & June \\
\hline One month & $1,985.9$ & $1,687.1$ & $4,980.7$ & $11,771.6$ & $13,874.1$ & $10,882.7$ \\
\hline Three months & $1,173.7$ & $2,245.9$ & $5,772.1$ & $6,219.1$ & $5,419.2$ & $8,331.2$ \\
\hline Total & $3,159.6$ & $3,933.0$ & $10,752.8$ & $17,990.7$ & $19,293.3$ & $19,216.9$ \\
\hline Variation (net) & $-\mathbf{3 4 0 . 4}$ & 773.4 & $6,819.8$ & $7,237.9$ & $1,302.6$ & -76.4 \\
\hline $\begin{array}{l}\text { Outstanding treasury certificates } \\
\text { (in percent of stock of broad money } \\
\text { at beginning of period) }\end{array}$ & 8.1 & 7.5 & 21.1 & 31.4 & 30.6 & 30.4 \\
\hline
\end{tabular}

Source: Bank of the Republic of Burundi.

1/ These figures do not include treasury certificates sold by the central bank in the open market. 
Table 34. Burundi: Distribution of Credit to the Economy, 1994-98

\begin{tabular}{|c|c|c|c|c|c|}
\hline & 1994 & 1995 & 1996 & 1997 & $\begin{array}{r}1998 \\
\text { June }\end{array}$ \\
\hline & \multicolumn{5}{|c|}{ (In millions of Burundi francs, unless otherwise specified) } \\
\hline Short term & $35,757.6$ & $33,457.4$ & $40,764.5$ & $44,263.7$ & $52,922.0$ \\
\hline \multicolumn{5}{|l|}{ Of which } & 78.0 \\
\hline Agricultural activities & 476.7 & 434.5 & $\ldots$ & $\ldots$ & $\ldots$ \\
\hline Industrial activities & $4,623.8$ & $4,140.3$ & $\ldots$ & $\ldots$ & $\ldots$ \\
\hline Trade & $13,391.6$ & $15,803.6$ & $\ldots$ & $\ldots$ & $\ldots$ \\
\hline Of which: coffee exports & $3,313.7$ & $3,519.5$ & $9,930.2$ & $8,607.7$ & $6,195.3$ \\
\hline Medium term & $7,861.5$ & $7,989.2$ & $8,195.3$ & $6,852.4$ & $7,501.7$ \\
\hline $\begin{array}{l}\text { In percent of total } \\
\text { Of which }\end{array}$ & 15.4 & 16.1 & 14.3 & 11.6 & 11.1 \\
\hline Agricultural activities & 170.4 & 118.2 & $\cdots$ & $\cdots$ & ... \\
\hline Civil engineering & $1,595.2$ & $1,693.5$ & $\ldots$ & $\ldots$ & ... \\
\hline Industrial activities & $1,730.9$ & $1,081.2$ & $\ldots$ & $\ldots$ & $\ldots$ \\
\hline Trade & $1,091.5$ & 968.8 & $\ldots$ & $\ldots$ & $\ldots$ \\
\hline Transport and transit & 608.0 & 443.2 & $\ldots$ & $\cdots$ & $\ldots$ \\
\hline Long term & $7,443.3$ & $8,251.8$ & $8,216.3$ & $7,763.4$ & $7,452.8$ \\
\hline $\begin{array}{l}\text { In percent of total } \\
\text { Of which }\end{array}$ & 14.6 & 16.6 & 14.4 & 13.2 & 10.9 \\
\hline Agricultural activities & 296.1 & 288.3 & $\cdots$ & $\ldots$ & ... \\
\hline Civil engineering & $3,641.5$ & $5,132.4$ & $\ldots$ & $\ldots$ & $\ldots$ \\
\hline Other services & 299.9 & 70.2 & $\ldots$ & $\ldots$ & $\ldots$ \\
\hline Trade & 574.9 & 413.0 & $\ldots$ & $\ldots$ & $\ldots$ \\
\hline \multirow[t]{2}{*}{ Total 1/ } & $51,062.4$ & $49,698.4$ & $57,176.1$ & $58,879.5$ & $67,876.5$ \\
\hline & \multicolumn{5}{|c|}{ (In percent of total credit) } \\
\hline Agricultural activities & 1.9 & 1.7 & $\ldots$ & $\ldots$ & $\ldots$ \\
\hline Civil engineering and other services & 10.8 & 13.9 & $\ldots$ & $\ldots$ & $\ldots$ \\
\hline Industrial activities & 12.4 & 10.5 & $\ldots$ & $\ldots$ & ... \\
\hline Trade & 29.5 & 34.6 & $\ldots$ & $\ldots$ & ... \\
\hline Transport and transit & 1.2 & 0.9 & $\ldots$ & $\ldots$ & $\ldots$ \\
\hline
\end{tabular}

Source: Bank of the Republic of Burundi.

1/ Includes credit provided by commercial banks and other financial institutions. 
Table 35. Burundi: Structure of Interest Rates, 1994-98

(In percent per annum)

\begin{tabular}{|c|c|c|c|c|c|c|}
\hline & \multirow{2}{*}{1994} & \multirow{2}{*}{1995} & \multirow{2}{*}{1996} & \multirow{2}{*}{1997} & \multicolumn{2}{|c|}{1998} \\
\hline & & & & & Mar. & June \\
\hline \multicolumn{7}{|l|}{ Lending rates } \\
\hline Short-term loans & 16.4 & 15.9 & 15.4 & 16.1 & 17.0 & 17.6 \\
\hline Export credits & 8.6 & 10.1 & 12.0 & 12.1 & 12.0 & 12.5 \\
\hline Cash-flow credits & 17.9 & 16.8 & 16.6 & 17.1 & 17.9 & 18.3 \\
\hline Medium-term loans & 16.1 & 15.4 & 15.3 & 15.8 & 16.2 & 16.8 \\
\hline Housing construction & 16.9 & 16.6 & 16.4 & 16.7 & 17.3 & 17.5 \\
\hline Other medium-term loans & 15.9 & 14.8 & 14.9 & 15.5 & 15.8 & 16.6 \\
\hline Long-term loans & 14.6 & 15.0 & 15.0 & 14.5 & 14.5 & 14.6 \\
\hline Housing construction & 13.4 & 14.0 & 13.6 & 14.2 & 14.2 & 16.2 \\
\hline Other long-term loans & 14.8 & 15.3 & 15.1 & 14.5 & 14.5 & 14.5 \\
\hline Rediscount rate of the central bank & 10.0 & 10.0 & 10.0 & 12.0 & 12.0 & 12.0 \\
\hline \multicolumn{7}{|l|}{ Deposit rates } \\
\hline Demand deposits & 2.4 & 4.3 & 3.8 & 4.8 & 5.2 & 5.8 \\
\hline Passbook savings & 8.0 & 8.0 & 8.1 & 8.0 & 8.0 & 8.0 \\
\hline \multicolumn{7}{|l|}{ Time deposits } \\
\hline Less than 1 month & 9.3 & 9.0 & 8.7 & 8.7 & 9.2 & 9.2 \\
\hline Up to 12 months & 10.1 & 9.5 & 9.1 & 9.3 & 9.4 & 9.4 \\
\hline Up to 24 months & 12.5 & 12.1 & 8.6 & 8.5 & 8.9 & 8.5 \\
\hline More than 24 months & 11.8 & 12.9 & 8.5 & 8.5 & 8.5 & 8.5 \\
\hline \multicolumn{7}{|l|}{ Advance-notice deposits } \\
\hline Up to 1 month & 6.5 & 6.3 & 6.4 & 5.0 & 5.0 & 5.0 \\
\hline Up to 12 months & 5.5 & 7.5 & 9.6 & 5.5 & 5.5 & 5.5 \\
\hline \multicolumn{7}{|l|}{ Savings bonds } \\
\hline Up to 1 month & 11.3 & 9.1 & $\ldots$ & 9.1 & 10.5 & 10.5 \\
\hline Up to 12 months & 11.2 & 9.8 & 9.3 & 9.6 & 9.7 & 9.8 \\
\hline More than 12 months & 11.2 & $\ldots$ & $\ldots$ & $\ldots$ & $\ldots$ & $\ldots$ \\
\hline
\end{tabular}

Source: Bank of the Republic of Burundi. 
Table 36. Burundi: Balance of Payments, 1994-98

(In millions of U.S. dollars)

\begin{tabular}{|c|c|c|c|c|c|}
\hline & 1994 & 1995 & 1996 & $\begin{array}{c}1997 \\
\text { Prel. }\end{array}$ & $\begin{array}{c}1998 \\
\text { Proj. }\end{array}$ \\
\hline Trade balance & -91.9 & -63.0 & -59.9 & -10.6 & -53.2 \\
\hline Exports, f.o.b. & 80.7 & 112.5 & 40.1 & 87.3 & 49.3 \\
\hline Of which: coffee & 57.2 & 89.1 & 28.8 & 76.6 & 38.6 \\
\hline Imports, f.o.b & -172.6 & -175.6 & -100.0 & -97.9 & -102.6 \\
\hline Services (net) & -87.9 & -93.6 & -40.8 & -40.4 & -42.0 \\
\hline Nonfactor services (net) & -76.5 & -81.0 & -26.8 & -29.7 & -32.2 \\
\hline Factor services (net) & -11.4 & -12.6 & -14.0 & -10.7 & -9.8 \\
\hline Private transfers (net) & 17.4 & 15.6 & 11.2 & 8.7 & 11.0 \\
\hline Current account (excluding official transfers) & -162.4 & -141.0 & -89.4 & -42.3 & -84.2 \\
\hline Official transfers & 143.8 & 136.1 & 49.8 & 50.9 & 50.9 \\
\hline Current account (including official transfers) & -18.6 & -4.9 & -39.6 & 8.6 & -33.3 \\
\hline Capital account & 62.4 & 7.3 & -43.4 & -39.8 & 8.0 \\
\hline Direct investment & -0.1 & 1.4 & 0.0 & 0.0 & 0.0 \\
\hline Medium- and long-term official loans (net) & 25.2 & 4.9 & 7.3 & 3.5 & 8.0 \\
\hline Disbursements & 50.8 & 40.3 & 46.1 & 26.0 & 30.0 \\
\hline Program loans & 2.2 & 0.0 & 0.0 & 0.0 & 0.0 \\
\hline Project loans & 48.5 & 40.3 & 46.1 & 26.0 & 30.0 \\
\hline Amortization & -25.6 & -35.4 & -38.8 & -22.6 & -22.0 \\
\hline Other capital and errors and omissions $1 /$ & 37.3 & 1.0 & -50.7 & -43.3 & 0.0 \\
\hline Overall balance & 43.8 & 2.4 & -83.0 & -31.3 & -25.3 \\
\hline Financing (increase in assets -) & -43.8 & -2.4 & 83.0 & 31.2 & 25.3 \\
\hline Change in net reserves & -47.9 & -11.8 & 58.3 & 15.6 & 9.1 \\
\hline Change in arrears (increase + ) & 4.1 & 9.4 & 24.7 & 15.7 & 16.2 \\
\hline Financing gap & 0.0 & 0.0 & 0.0 & 0.0 & 0.0 \\
\hline \multicolumn{6}{|l|}{ Memorandum items: } \\
\hline Trade balance/GDP (in percent) & -10.0 & -6.3 & -6.6 & -1.1 & -5.5 \\
\hline \multicolumn{6}{|l|}{ Current account (excluding official transfers)/GDP } \\
\hline (in percent) & -17.6 & -14.1 & -9.9 & -4.4 & -8.6 \\
\hline \multicolumn{6}{|l|}{ Current account (including official transfers)/GDP } \\
\hline (in percent) & -2.0 & -0.5 & -4.4 & 0.9 & -3.4 \\
\hline \multicolumn{6}{|l|}{ Gross official reserves } \\
\hline In U.S. dollars & 213.9 & 215.3 & 144.7 & 117.3 & 108.2 \\
\hline In months of imports, c.i.f. & 8.2 & 7.8 & 10.8 & 9.6 & 8.6 \\
\hline Net reserves (in months of imports, c.i.f.) & 7.8 & 8.1 & 9.5 & 8.1 & 6.9 \\
\hline \multirow{2}{*}{\multicolumn{6}{|c|}{ Scheduled debt service (in percent of exports }} \\
\hline & & & & & \\
\hline of goods and nonfactor services) & 39.6 & 39.3 & 105.9 & 36.3 & 57.9 \\
\hline Exchange rate (FBu/US\$; period average) & 252.7 & 249.8 & 302.8 & 352.4 & 435.2 \\
\hline
\end{tabular}

Sources: Burundi authorities; and Fund staff estimates and projections.

1/ Includes private loans, short-term capital, and errors and omissions. 
Table 37: Burundi: Composition of Exports, f.o.b., 1994-98

(Value in millions of U.S. dollars; volume and other units as indicated)

\begin{tabular}{|c|c|c|c|c|c|}
\hline & 1994 & 1995 & 1996 & $\begin{array}{r}1997 \\
\text { Est. }\end{array}$ & $\begin{array}{l}1998 \\
\text { Proj }\end{array}$ \\
\hline \multicolumn{6}{|l|}{ Coffee } \\
\hline Value & 57.2 & 89.2 & 28.8 & 76.6 & 38.6 \\
\hline Volume (tons) & 20,941 & 30,245 & 14,471 & 31,836 & 22,000 \\
\hline Unit value (US\$ per kg.) & 2.7 & 2.9 & 2.0 & 2.4 & 1.8 \\
\hline Unit value (cents per lb.) & 122.5 & 131.5 & 90.7 & 109.2 & 79.7 \\
\hline \multicolumn{6}{|l|}{ Tea } \\
\hline Value & 9.3 & 9.7 & 4.7 & 9.0 & 9.0 \\
\hline Volume (tons) & 5,575 & 6,795 & 3,412 & 6,330 & 6,500 \\
\hline Unit value (US\$ per kg.) & 1.7 & 1.4 & 1.4 & 1.4 & 1.4 \\
\hline \multicolumn{6}{|l|}{ Cotton } \\
\hline Value & 3.4 & 1.7 & 0.0 & 0.0 & 0.0 \\
\hline Volume (tons) & 2,130 & 889 & 0.0 & 0.0 & 0.0 \\
\hline Unit value (US\$ per kg.) & 1.6 & 1.9 & 0.0 & 0.0 & 0.0 \\
\hline \multicolumn{6}{|l|}{ Hides and skins } \\
\hline Value & 2.4 & 2.1 & 0.9 & 0.1 & 0.1 \\
\hline Volume (tons) & 839 & 664 & 355 & 179 & 179 \\
\hline Unit value (US\$ per $\mathrm{kg}$.) & 2.8 & 3.2 & 2.5 & 0.6 & 0.5 \\
\hline \multicolumn{6}{|l|}{ Other primary products } \\
\hline Value & 2.9 & 2.9 & 1.6 & 1.1 & 1.2 \\
\hline \multicolumn{6}{|l|}{ Manufactured products } \\
\hline Value & 5.4 & 7.0 & 4.2 & 0.5 & 0.5 \\
\hline \multicolumn{6}{|l|}{ Reexports } \\
\hline Value & 0.0 & 0.0 & 0.0 & 0.0 & 0.0 \\
\hline Total export value & 80.7 & 112.5 & 40.1 & 87.3 & 49.3 \\
\hline (percent change) & 9.2 & 39.5 & -64.4 & 117.4 & -43.5 \\
\hline
\end{tabular}

Sources: Burundi authorities; and Fund staff estimates and projections. 
Table 38. Burundi: Composition of Imports, c.i.f., 1994-98

(Values in millions of U.S. dollars; other units as indicated)

\begin{tabular}{|c|c|c|c|c|c|}
\hline & 1994 & 1995 & 1996 & $\begin{array}{r}1997 \\
\text { Est. }\end{array}$ & $\begin{array}{l}1998 \\
\text { Proj. }\end{array}$ \\
\hline \multicolumn{6}{|l|}{ Capital goods } \\
\hline Value & 80.9 & 87.6 & 48.5 & 45.3 & 48.8 \\
\hline (percent change) & 3.3 & 8.3 & -44.6 & -6.6 & 7.7 \\
\hline (percent change in volume) & 0.2 & -3.3 & -42.7 & 2.8 & 12.1 \\
\hline (percent change in unit value) & 3.1 & 12.0 & -3.4 & -9.1 & -3.9 \\
\hline \multicolumn{6}{|l|}{ Intermediate goods } \\
\hline Value & 55.3 & 62.4 & 38.1 & 33.3 & 34.9 \\
\hline (percent change) & -3.0 & 12.8 & -38.9 & -12.6 & 4.8 \\
\hline Petroleum products & 29.0 & 26.6 & 18.2 & 13.8 & 14.3 \\
\hline (percent change) & 21.8 & -8.3 & -31.6 & -24.2 & 3.6 \\
\hline (percent change in volume) & 29.0 & -15.1 & -42.3 & -19.8 & 50.4 \\
\hline (percent change in unit value) & -5.5 & 8.1 & 18.6 & -5.4 & -31.1 \\
\hline Other & 26.3 & 35.8 & 19.9 & 19.5 & 20.6 \\
\hline (percent change) & -20.8 & 36.1 & -44.4 & -2.0 & 5.7 \\
\hline (percent change in volume) & 22.9 & 33.2 & -45.9 & -4.8 & 10.0 \\
\hline (percent change in unit value) & 2.8 & 2.2 & 2.8 & 2.9 & -3.9 \\
\hline \multicolumn{6}{|l|}{ Consumption goods } \\
\hline Value & 87.4 & 82.9 & 36.8 & 44.1 & 44.9 \\
\hline (percent change) & 16.5 & -5.1 & -55.6 & 19.8 & 1.8 \\
\hline Food & 30.0 & 28.8 & 10.9 & 11.2 & 10.1 \\
\hline (percent change) & 61.3 & -4.0 & -62.2 & 2.8 & -9.8 \\
\hline (percent change in volume) & 53.1 & -11.6 & -66.3 & 15.3 & 2.8 \\
\hline (percent change in unit value) & 5.0 & 8.6 & 12.3 & -10.9 & -12.3 \\
\hline Other & 57.4 & 54.1 & 25.9 & 32.9 & 34.8 \\
\hline (percent change) & 1.8 & -5.7 & -52.1 & 27.0 & 5.8 \\
\hline (percent change in volume) & -1.3 & -15.8 & -50.4 & 39.7 & 10.1 \\
\hline (percent change in unit value) & 3.1 & 12.0 & -3.4 & -9.1 & -3.9 \\
\hline Other (adjustments) & 0.0 & 0.0 & 0.0 & 0.0 & 0.0 \\
\hline Total imports, c.i.f. & 223.6 & 232.9 & 123.4 & 122.7 & 128.6 \\
\hline (percent change) & 6.3 & 4.2 & -47.0 & -0.6 & 4.8 \\
\hline
\end{tabular}

Sources: Burundi authorities; and Fund staff estimates and projections. 
Table 39. Burundi: Services and Transfers, 1994-98

(In millions of U.S. doliars)

\begin{tabular}{|c|c|c|c|c|c|}
\hline & 1994 & 1995 & 1996 & $\begin{array}{r}1997 \\
\text { Est. }\end{array}$ & $\begin{array}{l}1998 \\
\text { Proj. }\end{array}$ \\
\hline Services (net) & -87.9 & -93.6 & -40.8 & -40.4 & -42.0 \\
\hline Nonfactor services (net) & -76.4 & -81.0 & -26.8 & -29.7 & -32.2 \\
\hline Credit & 14.5 & 16.4 & 10.1 & 8.3 & 7.6 \\
\hline Freight & 1.8 & 1.0 & 0.5 & 0.8 & 0.5 \\
\hline Passenger services & 1.3 & 1.0 & 0.9 & 0.8 & 0.5 \\
\hline Revenue from government services & 1.3 & 3.6 & 1.6 & 2.7 & 2.8 \\
\hline Travel & 1.9 & 1.4 & 1.2 & 0.6 & 0.3 \\
\hline Foreign governments & 7.2 & 8.4 & 4.4 & 2.6 & 2.7 \\
\hline Other & 0.9 & 0.9 & 1.5 & 0.8 & 0.8 \\
\hline Debit & -90.9 & -97.4 & -36.9 & -38.0 & -39.8 \\
\hline Freight (including insurance) & -35.8 & -30.6 & -13.8 & -19.8 & -20.7 \\
\hline Other freight & 0.0 & 0.0 & 0.0 & $\ldots$ & $\ldots$ \\
\hline Travel & -18.0 & -25.4 & -12.1 & -10.1 & -10.5 \\
\hline Government of Burundi (net) & -18.9 & -21.1 & -9.1 & -8.1 & -8.5 \\
\hline Studies & $\ldots$ & $\ldots$ & $\ldots$ & $\ldots$ & $\ldots$ \\
\hline Scholarships & $\cdots$ & $\ldots$ & $\ldots$ & $\ldots$ & $\ldots$ \\
\hline Technical assistance & -15.9 & -17.8 & $\ldots$ & $\ldots$ & $\ldots$ \\
\hline Other & -2.4 & -2.5 & -1.9 & $\ldots$ & ... \\
\hline Factor services (net) & -11.5 & -12.6 & -14.0 & -10.7 & -9.8 \\
\hline Credit (investment income) & 12.5 & 10.4 & 6.4 & 4.3 & 4.3 \\
\hline Debit & -24.0 & -23.0 & -20.4 & -15.0 & -14.1 \\
\hline Dividends & -3.4 & -3.1 & -1.5 & -0.6 & -0.6 \\
\hline $\begin{array}{l}\text { Interest payments } \\
\text { Of which }\end{array}$ & -13.6 & -15.3 & -14.4 & -12.1 & -11.0 \\
\hline Interest on public debt & -12.6 & -14.1 & -13.3 & -11.0 & -10.0 \\
\hline Labor income & -6.0 & -4.5 & -4.5 & -2.3 & -2.3 \\
\hline Property income & -1.0 & -0.1 & 0.0 & 0.0 & -0.2 \\
\hline Transfers (net) & 161.2 & 151.7 & 61.0 & 59.6 & 59.8 \\
\hline Private (net) & 17.4 & 15.6 & 11.2 & 8.7 & 11.0 \\
\hline Receipts & 18.3 & 17.5 & 12.1 & 9.8 & 12.1 \\
\hline Payments & -0.9 & -1.9 & -0.9 & -1.1 & -1.1 \\
\hline Official receipts (net) & 143.8 & 136.1 & 49.8 & 50.9 & 50.9 \\
\hline Credit & 144.7 & 137.1 & 50.4 & 51.4 & 51.4 \\
\hline SAC cofinancing $1 /$ & $\ldots$ & $\ldots$ & $\ldots$ & $\ldots$ & $\ldots$ \\
\hline Other current grants & $\cdots$ & $\cdots$ & $\ldots$ & $\ldots$ & $\ldots$ \\
\hline STABEX i/ & $\ldots$ & $\ldots$ & $\ldots$ & $\ldots$ & $\ldots$ \\
\hline Technical assistance & $\ldots$ & $\ldots$ & $\ldots$ & $\ldots$ & $\ldots$ \\
\hline Other current & $\ldots$ & $\ldots$ & $\ldots$ & $\ldots$ & $\ldots$ \\
\hline $\begin{array}{l}\text { Capital grants } \\
\text { Debit (contribution to international }\end{array}$ & $\ldots$ & $\ldots$ & $\ldots$ & $\ldots$ & $\ldots$ \\
\hline organizations) & -0.9 & -1.0 & -0.6 & -0.5 & -0.5 \\
\hline
\end{tabular}

Sources: Burundi authorities; and Fund staff estimates and projections.

1/ SAC is Structural Adjustment Credit, and STABEX is Stabilization System for Export Earnings. 
Table 40. Burundi: Structure, Volume, and Prices of International Trade, 1994-98

\begin{tabular}{|c|c|c|c|c|c|}
\hline & 1994 & 1995 & 1996 & $\begin{array}{c}1997 \\
\text { Est. }\end{array}$ & $\begin{array}{l}1998 \\
\text { Proj. }\end{array}$ \\
\hline & \multicolumn{5}{|c|}{ (In percent of total) } \\
\hline Exports, f.o.b. & 100.0 & 100.0 & 100.0 & 100.0 & 100.0 \\
\hline Coffee & 70.9 & 79.2 & 71.8 & 87.7 & 78.3 \\
\hline Tea & 11.5 & 8.6 & 11.5 & 10.3 & 18.3 \\
\hline Cotton & 4.3 & 1.5 & 0.0 & 0.0 & 0.0 \\
\hline Hides and skins & 3.0 & 1.9 & 2.2 & 0.1 & 0.2 \\
\hline Other primary products & 3.6 & 2.6 & 4.0 & 1.3 & 2.4 \\
\hline Manufactured products & 6.8 & 6.2 & 10.7 & 0.6 & 1.0 \\
\hline Reexports & 0.0 & 0.0 & 0.0 & 0.0 & 0.0 \\
\hline Imports, c.i.f. & 100.0 & 100.0 & 99.9 & 100.0 & 100.0 \\
\hline Intermediate goods & 24.7 & 26.8 & 30.8 & 27.1 & 27.1 \\
\hline Petroleum products & 13.0 & 11.4 & 14.8 & 11.3 & 11.1 \\
\hline Other & 11.8 & 15.4 & 16.0 & 15.8 & 16.0 \\
\hline Capital goods & 36.2 & 37.6 & 39.3 & 36.9 & 38.0 \\
\hline Consumer goods & 39.1 & 35.6 & 29.8 & 35.9 & 34.9 \\
\hline Foodstuffs & 13.4 & 12.4 & 8.8 & 9.1 & 7.9 \\
\hline \multirow[t]{2}{*}{ Nonfoodstuffs } & 25.7 & 23.2 & 21.0 & 26.8 & 27.0 \\
\hline & \multicolumn{5}{|c|}{ (Index, 1985 =100, unless otherwise indicated) } \\
\hline Export volume & 70.3 & 93.2 & 44.8 & 85.5 & 63.7 \\
\hline Export unit value (U.S. dollars) & 101.0 & 106.2 & 78.8 & 89.9 & 68.1 \\
\hline Import volume & 83.2 & 77.0 & 43.0 & 45.2 & 51.3 \\
\hline Import unit value (U.S. dollars) & 140.2 & 154.0 & 157.1 & 146.4 & 135.1 \\
\hline \multirow[t]{2}{*}{ Terms of trade } & 72.0 & 68.9 & 50.1 & 61.4 & 50.4 \\
\hline & \multicolumn{5}{|c|}{ (Annual percentage change) } \\
\hline Export volume & -24.9 & 32.7 & -51.9 & 90.8 & -25.5 \\
\hline Export unit value (U.S. dollars) & 45.5 & 5.1 & -25.8 & 14.0 & -24.2 \\
\hline Import volume & -2.2 & -7.4 & -44.2 & 5.1 & 13.5 \\
\hline Import unit value (U.S. dollars) & 2.2 & 9.9 & 2.0 & -6.8 & .7 .7 \\
\hline Terms of trade & 42.3 & -4.3 & -27.2 & 22.3 & -17.9 \\
\hline
\end{tabular}

Sources: Burundi authorities; and Fund staff estimates and projections. 
Table 41. Burundi: Direction of Trade, 1994-97

\begin{tabular}{|c|c|c|c|c|}
\hline & \multicolumn{2}{|c|}{ (In percent of total) } & \multirow[b]{2}{*}{1996} & \multirow[b]{2}{*}{$\begin{array}{r}1997 \\
\text { Est. }\end{array}$} \\
\hline & 1994 & 1995 & & \\
\hline Exports, f.o.b. & 100.0 & 100.0 & 100.0 & 100.0 \\
\hline European Union countries & 39.0 & 38.4 & 20.0 & 64.8 \\
\hline Belgium & 1.4 & 2.1 & 2.6 & 16.0 \\
\hline France & 3.5 & 1.8 & 0.0 & 0.1 \\
\hline Germany & 7.8 & 3.9 & 2.2 & 21.4 \\
\hline Italy & 1.2 & 1.2 & 0.8 & 0.2 \\
\hline United Kingdom & 24.1 & 28.4 & 13.4 & 27.0 \\
\hline Netherlands & 0.7 & 1.0 & 0.7 & 0.0 \\
\hline Spain, Portugal, and Denmark & 0.3 & 0.0 & 0.3 & 0.0 \\
\hline Others & 0.0 & 0.0 & 0.0 & 0.1 \\
\hline African countries & 10.1 & 12.6 & 24.5 & 2.7 \\
\hline Kenya & 4.7 & 5.2 & 3.8 & 0.0 \\
\hline Rwanda & 1.3 & 1.9 & 9.3 & 0.4 \\
\hline Uganda & 0.6 & 1.0 & 0.2 & 0.0 \\
\hline Zaire & 2.0 & 0.5 & 0.3 & 0.0 \\
\hline Others & 1.5 & 4.0 & 10.9 & 2.3 \\
\hline Finland & 0.0 & 0.0 & 0.0 & 0.0 \\
\hline Japan & 0.0 & 0.0 & 0.0 & 0.0 \\
\hline United States & 0.1 & 0.2 & 0.0 & 0.9 \\
\hline Others & 50.8 & 48.8 & 55.5 & 31.6 \\
\hline Imports, c.i.f. & 100.0 & 100.0 & 100.0 & 100.0 \\
\hline European Union countries & 42.7 & 50.8 & 45.9 & 46.2 \\
\hline Belgium & 13.9 & 15.4 & 15.3 & 18.2 \\
\hline France & 11.4 & 10.1 & 8.6 & 9.2 \\
\hline Germany & 5.2 & 8.1 & 8.8 & 6.6 \\
\hline Italy & 3.0 & 3.9 & 3.9 & 3.4 \\
\hline Netherlands & 4.8 & 5.3 & 4.8 & 4.3 \\
\hline United Kingdom, and Ireland & 2.4 & 4.0 & 2.5 & 2.5 \\
\hline Others & 2.0 & 3.9 & 1.9 & 2.0 \\
\hline African countries & 18.7 & 16.4 & 17.3 & 22.1 \\
\hline Kenya & 4.4 & 4.1 & 3.7 & 4.3 \\
\hline Malawi & 0.3 & 0.5 & $\ldots$ & 0.6 \\
\hline Tanzania & 2.5 & 2.7 & 3.2 & 2.5 \\
\hline Zaire & 1.2 & 0.8 & 0.8 & 0.2 \\
\hline Zambia & 3.3 & 3.3 & 3.5 & 5.5 \\
\hline Zimbabwe & 2.2 & 1.8 & 1.3 & 2.3 \\
\hline Others & 4.8 & 3.2 & 4.8 & 6.7 \\
\hline Iran, Islamic Republic of & 7.2 & 1.3 & $\ldots$ & 0.7 \\
\hline Japan & 7.9 & 5.7 & 6.7 & 6.3 \\
\hline Other Asian countries & 15.3 & 19.0 & 22.6 & 20.6 \\
\hline United States & 3.7 & 4.9 & 4.6 & 1.9 \\
\hline Others & 4.5 & 1.9 & 2.9 & 2.2 \\
\hline
\end{tabular}

Sources: Burundi authorities; and Fund staff estimates. 
Table 42. Burundi: Trade Flows with the Preferential Trade Area Countries, 1994-97 1/ (In millions of Burundi francs)

\begin{tabular}{|c|c|c|c|c|c|c|c|c|}
\hline & \multicolumn{2}{|c|}{1994} & \multicolumn{2}{|c|}{1995} & \multicolumn{2}{|c|}{1996} & \multicolumn{2}{|c|}{1997} \\
\hline & Exports & Imports & Exports & Imports & Exports & Imports & Exports & Imports \\
\hline Tanzania & 346.5 & $1,404.5$ & $1,126.7$ & $1,562.0$ & $1,182.3$ & $1,179.2$ & 0.5 & $1,077.1$ \\
\hline Kenya & $1,412.5$ & $2,481.1$ & $1,362.5$ & $2,388.3$ & 425.1 & $1,391.6$ & 13.0 & $1,869.2$ \\
\hline Kenya & 406.0 & 96.7 & 487.2 & 129.1 & $1,045.0$ & 73.2 & 133.4 & 321.0 \\
\hline Zambia & 15.9 & $1,835.5$ & 42.0 & $1,931.7$ & 4.2 & $1,297.6$ & 0.0 & $2,382.3$ \\
\hline Djibouti & 0.0 & 0.0 & 0.0 & 2.9 & 0.0 & 31.1 & 0.0 & 18.4 \\
\hline Zimbabwe & $\$ 9.3$ & $1,256.3$ & 0.0 & $1,064.5$ & 56.4 & 491.9 & 0.0 & 988.8 \\
\hline Uganda & 192.4 & 0.0 & 235.7 & 161.8 & 23.6 & 33.1 & 0.0 & 0.0 \\
\hline Malawi & 0.0 & 170.3 & 0.0 & 317.4 & 0.0 & 12.7 & 0.0 & 244.5 \\
\hline Ethiopia & 0.0 & 0.0 & 0.0 & 2.1 & 0.0 & 0.0 & 0.0 & 52.6 \\
\hline Mauritius & 0.0 & 0.0 & 0.0 & 154.3 & 0.0 & 214.3 & 0.0 & 180.7 \\
\hline Lesotho & 0.0 & 0.0 & 0.0 & 0.0 & 0.0 & 0.0 & 0.0 & 0.0 \\
\hline Swaziland & 0.0 & 0.0 & 0.0 & 428.6 & 0.0 & 34.6 & 0.0 & 109.9 \\
\hline Sudan & 0.0 & 0.0 & 0.0 & 0.0 & 0.0 & 0.0 & 215.8 & 0.0 \\
\hline Mozambique & 0.0 & 0.0 & 0.0 & 2.1 & 0.0 & 0.0 & 0.0 & 0.0 \\
\hline Angola & 0.0 & 0.0 & 0.0 & 0.0 & 0.0 & 0.0 & 107.9 & 32.4 \\
\hline Comoros & 0.0 & 0.0 & 0.0 & 0.0 & 0.0 & 0.0 & 0.0 & 0.0 \\
\hline Somalia & 0.0 & 0.0 & 0.0 & 0.0 & 0.0 & 0.0 & 107.9 & 0.0 \\
\hline Total & $2,432.6$ & $7,244.4$ & $3,254.1$ & $8,144.8$ & $2,736.6$ & $4,759.3$ & 578.5 & $7,276.9$ \\
\hline
\end{tabular}

Source: Bank of the Republic of Burundi.

1/ Preferential Trade Area for Eastern and Southern Africa. 
Table 43. Outstanding Medium- and Long-Term Official External Debt, 1994-98

(In millions of U.S. dollars; end of year, unless otherwise specified)

\begin{tabular}{|c|c|c|c|c|c|}
\hline & 1994 & 1995 & 1996 & $\begin{array}{r}1997 \\
\text { Est. }\end{array}$ & $\begin{array}{l}1998 \\
\text { Proj. }\end{array}$ \\
\hline Multilateral debt $1 /$ & 886.1 & 949.5 & 936.4 & 946.0 & 960.1 \\
\hline IDA & 553.6 & 593.5 & 595.4 & 606.0 & 610.9 \\
\hline African Development Bank & 28.0 & 26.7 & 22.7 & 20.4 & 16.7 \\
\hline African Development Fund & 191.9 & 202.3 & 207.4 & 212.3 & 220.2 \\
\hline Arab Bank for Economic Development in Africa & 12.9 & 13.1 & 11.8 & 9.2 & 7.1 \\
\hline Arab League & 0.6 & 0.6 & 0.5 & 0.4 & 0.3 \\
\hline European Investment Bank & 16.4 & 18.5 & 15.3 & 14.8 & 14.6 \\
\hline European Union & 49.1 & 57.5 & 50.8 & 50.0 & 49.4 \\
\hline International Fund for Agricultural Development & 18.3 & 20.9 & 17.8 & 19.7 & 29.3 \\
\hline Organization of Petroleum Exporting Countries & 13.8 & 14.8 & 13.3 & 11.9 & 10.2 \\
\hline IFC & 0.5 & 0.5 & 0.4 & 0.4 & 0.4 \\
\hline United Nations Development Program & 1.0 & 1.1 & 1.0 & 1.0 & 1.0 \\
\hline Bilateral Debt $1 /$ & 158.9 & 167.9 & 157,3 & 152.4 & 147.5 \\
\hline Abu Dhabi Fund for Arab Economic Development & 2.4 & 2.1 & 1.4 & 1.4 & 0.9 \\
\hline Austria & 8.5 & 13.7 & 11.9 & 15.5 & 15.5 \\
\hline China, People's Republic of & 18.7 & 21.4 & 19.4 & 15.5 & 15.1 \\
\hline France & 59.3 & 64.4 & 61.0 & 59.3 & 58.5 \\
\hline Italy (Mediocredito) & 1.7 & 1.6 & 1.3 & 1.3 & 1.3 \\
\hline Japan & 32.6 & 32.1 & 28.8 & 28.5 & 28.5 \\
\hline Kuwait & 17.5 & 15.6 & 15.9 & 14.3 & 12.7 \\
\hline Saudi Arabia & 18.2 & 17.0 & 17.6 & 16.6 & 15.1 \\
\hline Suppliers' credit $1 /$ & 2.2 & 1.8 & 0.8 & 0.8 & 0.8 \\
\hline Total outstanding debt (excluding arrears) & $1,047.2$ & $1,119.1$ & $1,094.5$ & $1,099.2$ & $1,108.3$ \\
\hline Arrears & 4.1 & 13.5 & 38.2 & 53.9 & 70.0 \\
\hline Use of Fund resources & 57.5 & 50.8 & 42.5 & 29.9 & 21.9 \\
\hline Total debt & $1,108.8$ & $1,183.4$ & $1,175.2$ & $1,183.0$ & $1,200.3$ \\
\hline \multicolumn{6}{|l|}{ Memorandum item: } \\
\hline Debt/GDP ratio (in percent) & 120.1 & 118.3 & 130.5 & 123.6 & 122.9 \\
\hline
\end{tabular}

Sources: Burundi authorities; and Fund staff estimates and projections.

1/ Excluding stock of arrears. 
Table 44. Burundi: Scheduled Debt Service, Principal, on Medium- and Long-Term

Official External Debt, 1994-98

(In millions of U.S. dollars)

\begin{tabular}{|c|c|c|c|c|c|}
\hline & 1994 & 1995 & 1996 & $\begin{array}{r}1997 \\
\text { Est. }\end{array}$ & $\begin{array}{l}1998 \\
\text { Proj. }\end{array}$ \\
\hline Multilateral debt & 10.6 & 15.4 & 17.0 & 15.0 & 16.0 \\
\hline IDA & 2.1 & 3.2 & 3.4 & 3.6 & 5.1 \\
\hline African Development Bank & 2.9 & 4.0 & 5.2 & 4.3 & 3.7 \\
\hline African Development Fund & 0.9 & 1.9 & 1.9 & 1.7 & 2.1 \\
\hline Arab League & 0.0 & 0.1 & 0.1 & 0.1 & 0.1 \\
\hline Arab Bank for Economic Development in Africa & 2.2 & 2.6 & 2.6 & 2.3 & 2.1 \\
\hline European Investment Bank & 0.0 & 0.1 & 0.0 & 0.2 & 0.2 \\
\hline European Union & 0.3 & 0.8 & 0.8 & 0.7 & 0.6 \\
\hline International Fund for Agricultural Development & 0.1 & 0.8 & 0.7 & 0.4 & 0.4 \\
\hline Organization of Petroleum Exporting Countries & 2.1 & 1.9 & 2.1 & 1.7 & 1.7 \\
\hline Other multilateral debt & 0.0 & 0.0 & 0.1 & $\ldots$ & $\ldots$ \\
\hline Bilateral debt & 6.0 & 8.1 & 10.9 & 7.7 & 6.0 \\
\hline Abu Dhabi Fund for Arab Economic Development & 0.6 & 0.6 & 0.6 & 0.4 & 0.4 \\
\hline Austria & 0.0 & 0.0 & 0.0 & $\ldots$ & $\ldots$ \\
\hline Belgium & 0.0 & 0.0 & 0.0 & $\ldots$ & $\ldots$ \\
\hline China & 1.4 & 2.3 & 4.0 & 2.4 & 1.8 \\
\hline France & 1.3 & 1.8 & 1.9 & 1.7 & 1.6 \\
\hline Italy (Mediocredito) & 0.0 & 0.0 & 0.0 & $\ldots$ & $\ldots$ \\
\hline Japan & 0.0 & 0.0 & 0.0 & 0.3 & $\ldots$ \\
\hline Kuwait & 1.4 & 2.0 & 2.1 & 1.8 & 0.7 \\
\hline Netherlands & 0.0 & 0.0 & 0.0 & $\ldots$ & $\ldots$ \\
\hline Saudi Arabia & 1.3 & 1.4 & 2.3 & 1.1 & 1.5 \\
\hline Russia & 0.0 & 0.0 & 0.0 & & \\
\hline Financial institutions & 0.0 & 0.0 & 0.0 & $\ldots$ & $\ldots$ \\
\hline Suppliers' credits & 0.1 & 0.0 & 0.0 & $\cdots$ & ... \\
\hline Other (unallocated) & 8.9 & 11.9 & 10.8 & $\cdots$ & $\ldots$ \\
\hline Total disbursed debt & 25.6 & 35.4 & 38.7 & 22.7 & 22.0 \\
\hline Use of Fund credit & 6.2 & 9.1 & 8.7 & 8.1 & 8.0 \\
\hline Total, including Fund credit & 31.8 & 44.5 & 47.4 & 30.8 & 30.0 \\
\hline \multicolumn{6}{|l|}{ Memorandum item: } \\
\hline Exchange rate (FBu/US\$; period average) & 252.7 & 249.8 & 302.8 & 352.4 & 435.2 \\
\hline
\end{tabular}

Sources: Burundi authorities; and Fund staff estimates and projections. 
Table 45. Burundi: Scheduled Debt Service, Interest, on Medium- and Long-Term Official External Debt, 1994-98

(In millions of U.S. dollars, unless otherwise specified)

\begin{tabular}{|c|c|c|c|c|c|}
\hline & 1994 & 1995 & 1996 & $\begin{array}{r}1997 \\
\text { Est. }\end{array}$ & $\begin{array}{l}1998 \\
\text { Proj. }\end{array}$ \\
\hline Multilateral debt & 8.9 & 11.5 & 10.7 & 8.4 & 7.7 \\
\hline IDA & 3.9 & 4.1 & 4.2 & 3.9 & 3.9 \\
\hline African Development Bank & 2.0 & 3.3 & 2.8 & 1.8 & 1.2 \\
\hline African Development Fund & 1.3 & 2.2 & 2.0 & 1.5 & 1.7 \\
\hline Arab Bank for Economic Development in Africa & 0.7 & 0.7 & 0.5 & 0.4 & 0.2 \\
\hline European Investment Bank & 0.4 & 0.3 & 0.3 & 0.4 & 0.1 \\
\hline European Union & 0.3 & 0.4 & 0.4 & 0.2 & 0.3 \\
\hline International Fund for Agricultural Development & 0.1 & 0.2 & 0.2 & 0.1 & 0.2 \\
\hline Organization of Petroleum Exporting Countries & 0.2 & 0.3 & 0.2 & 0.2 & 0.1 \\
\hline Other multilateral debt & 0.0 & 0.0 & 0.1 & $\ldots$ & $\ldots$ \\
\hline Bilateral debt & 2.2 & 2.5 & 2.7 & 2.6 & 2.1 \\
\hline Abu Dhabi Fund for Arab Economic Development & 0.1 & 0.1 & 0.1 & $\ldots$ & 0.0 \\
\hline France & 1.0 & 1.2 & 1.6 & 1.5 & 1.1 \\
\hline Italy (Mediocredito) & 0.0 & 0.0 & 0.0 & $\ldots$ & $\ldots$ \\
\hline Japan & 0.3 & 0.3 & 0.3 & 0.3 & 0.3 \\
\hline Kuwait & 0.4 & 0.6 & 0.5 & 0.4 & 0.3 \\
\hline Saudi Arabia & 0.4 & 0.3 & 0.4 & 0.4 & 0.3 \\
\hline Financial institutions & 0.1 & 0.1 & 0.0 & $\cdots$ & ... \\
\hline Suppliers' credits & 0.0 & 0.0 & 0.0 & $\cdots$ & ... \\
\hline Total disbursed debt & 11.2 & 14.1 & 13.4 & 11.0 & 10.0 \\
\hline Use of Fund credit & 1.0 & 1.2 & 1.0 & 1.1 & 0.9 \\
\hline Total, including Fund credit & 12.2 & 15.3 & 14.4 & 12.1 & 10.9 \\
\hline \multicolumn{6}{|l|}{ Memorandum item: } \\
\hline Exchange rate (FBu/US\$; period average) & 252.7 & 249.8 & 302.8 & 352.4 & 435.2 \\
\hline
\end{tabular}

Sources: Burundi authorities; and Fund staff estimates and projections. 
Table 46. Burundi: Outstanding Stock of External Debt and

Average Terms on Existing Loans, 1994-98

(In millions of U.S. dollars, unless otherwise specified)

\begin{tabular}{lrrrrr}
\hline & 1994 & 1995 & 1996 & $\begin{array}{r}1997 \\
\text { Est. }\end{array}$ & $\begin{array}{r}1998 \\
\text { Proj. }\end{array}$ \\
\hline Total debt (including IMF; excluding arrears) & $1,104.7$ & $1,169.9$ & $1,137.0$ & $1,129.1$ & $1,130.2$ \\
& $1,047.2$ & $1,119.1$ & $1,094.5$ & $1,099.2$ & $1,108.3$ \\
Total debt (excluding IMF and arrears) & & & & & \\
$\begin{array}{l}\text { Of which } \\
\quad \text { Multilateral loans }\end{array}$ & 886.1 & 949.5 & 936.4 & 946.0 & 960.0 \\
$\quad$ Bilateral loans & 161.1 & 169.7 & 158.1 & 153.2 & 148.3 \\
$\begin{array}{l}\text { Average interest rate } \\
\text { (in percent) }\end{array}$ & 1.2 & 1.5 & 1.3 & 1.1 & 1.0 \\
$\begin{array}{l}\text { Average maturity (in years) } \\
\text { Average grace period (in years) }\end{array}$ & 37.0 & 37.0 & 37.0 & 37.0 & 37.0 \\
Grant element (in percent) & 8.5 & 8.5 & 8.5 & 8.5 & 8.5 \\
\hline
\end{tabular}

Sources: Burundi authorities; IBRD, Debt Reporting System; and Fund staff estimates and projections. 
Figure 1. Burundi: Selected Economic and Financial Indicators, 1994-99
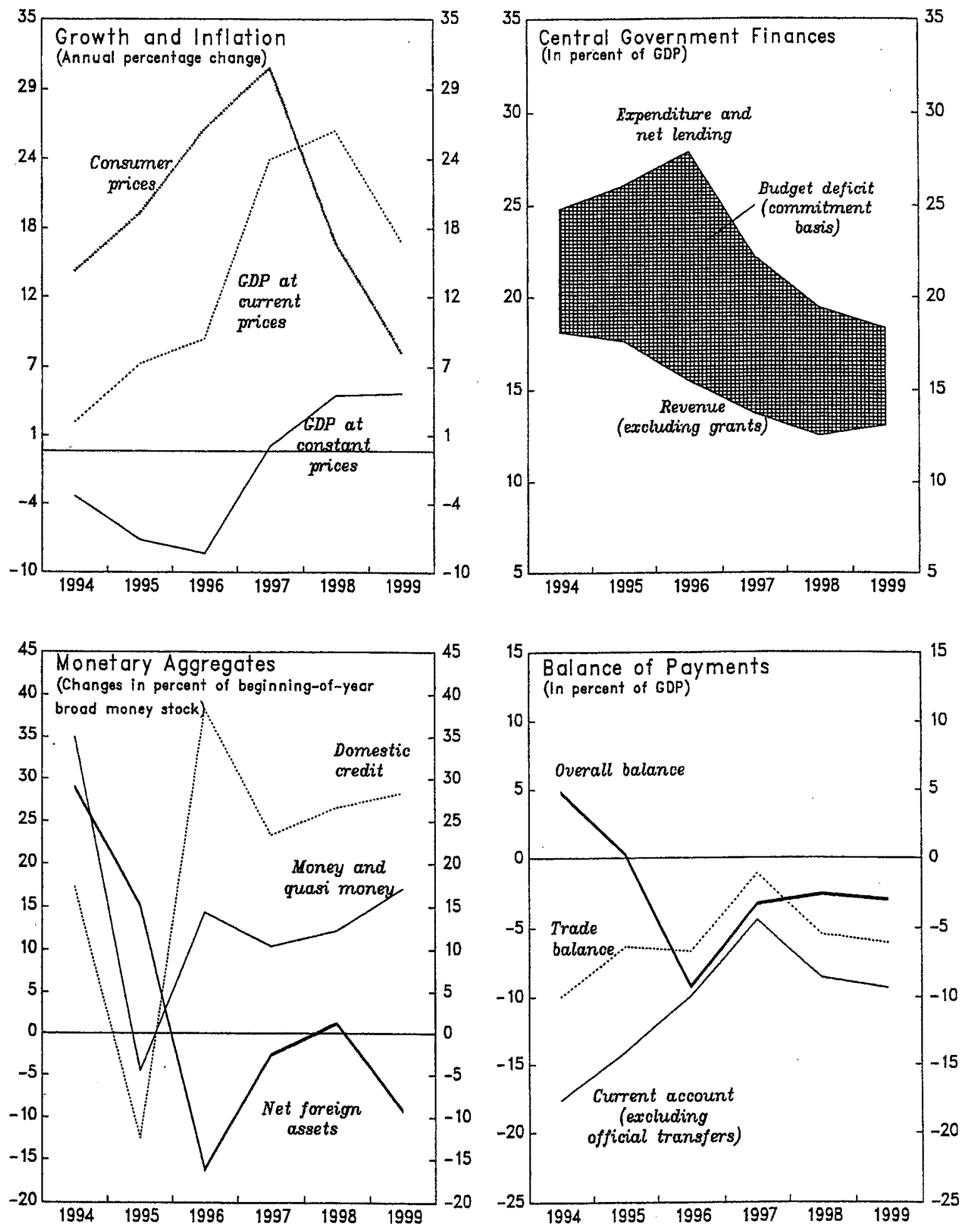

Sources: Burundi authorities; and Fund st off estimates and projections. 
Figure 2. Burundi: Selected Exchange Rate Indices, Jan. 1990-Aug. 1998

( $1990=100$; foreign currency per Burundi franc)
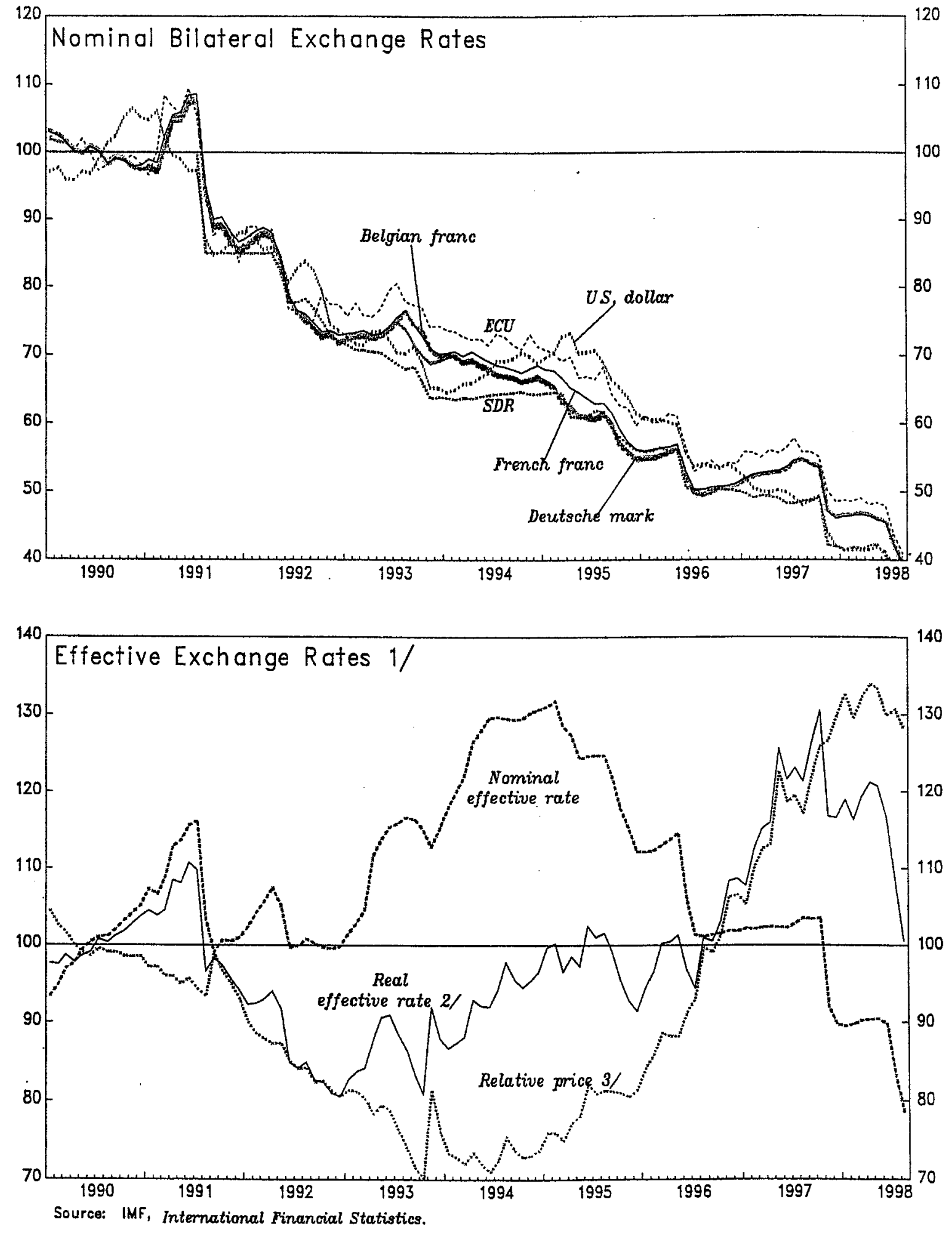

$1 /$ Information Notice System multilateral weights bosed on the geographical pottern of trade and fourism, including third markets.

2/Based on relotive consumer prices.

$3 /$ The ratio of the domestic price index to the weighted average of the partner country indices. 


\section{Burundi: Summary of Exchange and Trade System}

(At end-October 1998)

\section{Exchange rate system}

1. The Burundi franc was pegged to the SDR until April 1, 1992, when a new foreign exchange system was introduced. Since then, the Burundi franc has been pegged to a basket of currencies that reflects the pattern of Burundi's international trade. On October 31, 1998, the official exchange rate for the Burundi franc was FBu 499 per US\$1.

\section{Trade and payment system}

2. During the 1992-93 period, Burundi made progress in liberalizing its exchange and trade system. The authority for implementing foreign exchange regulations was delegated to commercial banks, allowing them to hold foreign exchange equivalent to up to 10 percent of their capital, and to manage freely their foreign assets. Holdings above the ceiling have to be approved by the Bank of the Republic of Burundi (BRB). Commercial banks are authorized to buy and sell foreign exchange at rates that they set freely within a spread of 1 percent below and above the central rate quoted by the BRB.

3. An open general licensing system was established in May 1992. In parallel, import licenses were replaced by an import declaration for statistical purposes.

4. Export licenses were replaced in May 1992 by an export declaration for statistical purposes, and exporters of nontraditional products were allowed to retain up to 30 percent of their export proceeds. ${ }^{1}$ In August 1992, a free trade zone was established, granting tax and customs duty exemptions for enterprises that elect to operate under this regime. In addition, at the start of the 1992/93 coffee crop year, coffee exporters were authorized to borrow in foreign exchange.

5. In July 1992, the limits on transfers of foreign nationals' incomes earned in Burundi were raised from 60 percent to 70 percent. A higher limit of 80 percent was introduced for expatriates working for firms that have at least 50 percent of their turnover accounted for by exports.

6. In November 1992, banks were authorized to open foreign accounts for enterprises established under the free trade zone regime, to be funded by the surrender of foreign exchange earnings. At the same time, Burundi nationals residing abroad were authorized to hold foreign exchange accounts at Burundi banks, to be funded from transfers from their incomes.

${ }^{1}$ This limit was raised to 50 percent in November 1996. 
7. In December 1995, the two remaining exchange restrictions (namely, limits on the transfer of rental income and on the granting of travel allowances) were removed.

8. In 1997, the authorities introduced restrictions on current payments and transfers in the face of dwindling foreign exchange reserves. In November 1997, the following restrictions were introduced: (i) importers were required to provide unremunerated advance deposits equivalent to 25 percent of the value of imports; (ii) the imports of luxury goods were negative listed; (iii) the provision of foreign exchange for transfers of income from rents, the sale of real estate, travel and study allowances, and outward direct investment, was suspended; and (iv) all foreign exchange operations were centralized at the BRB. These restrictions were further reinforced in July 1998 with the following measures: (i) the required advance import deposit was raised to 50 percent, and the deposits were shifted from commercial banks to the BRB; (ii) the negative list on imports was expanded; and (iii) the task of verifying import declarations and payments was shifted from commercial banks to the BRB.

9. The Burundi franc depreciated against the U.S. dollar by about 37 percent between December 1996 and June 1998 because of weak exports and a sharp decline in external aid. Despite this strong depreciation of the bilateral nominal exchange rate, the real effective exchange rate appreciated by 8 percent during the same period, reflecting mainly Burundi's inflation differential with its trading partners. The authorities, however, have recently adopted more active exchange rate policy to avoid further erosion of external competitiveness. Subsequently, the real effective exchange rate depreciated by 13 percent between June and September 1998. 
Burundi: Summary of the Tax System, End-June 1998

(All amounts in Burundi francs)

$\operatorname{Tax}$

Nature of Tax

Exemptions and Deductions

Rates

1. Tax on income and profits

1.1 Tax on companies (Tax Code II, Impôt sur les revenus professionnels, Arts. 27-94).
Annual tax on all net profits received by Burundi and foreign companies from Burundi sources. Realized capital gains are included in the profits. The purchase price of the assets may be adjusted so as to exclude gains that result from increases in the general price level. However, gains resulting from revaluation are subject to taxes if these gains are simply stated. Appreciation gains that have materialized are also subject to taxes.
Companies benefiting from the Investment Code are taxed accordingly.

Exemptions:

- Incomes covered by an international convention ratified by Burundi are exempt.

- Cooperative companies are exempt.

- Temporary exemptions for a period of ten years are given to enterprises operating in the free trade zone.

- Leasing and hire-purchase companies are exempt during a period spanning up to eight years.

Deductions:

Export companies can deduct 50 percent of their export profits.

1.2 Tax on rental income (Tax Code II, Impôt sur les revenus locatifs, Arts. 4-12).
Annual schedular tax on rental in- Exemptions:

come obtained from property

located in Burundi. It applies also to - Central government, local housing allowances paid to em. ployees occupying their own house The tax on rental income from property has been transferred to the municipality of Bujumbura (Law No. $1 / 001$ of $2 / 2 / 84$ ), and to the Communes (Law No. 1/003 of 1/8/87). governments, and decentralized central government units are exempt.

- Rental income are exempted under international agreement ratified by Burundi.

- New buildings are exempt up to four years maximum as follows (Decree Law No. 1/19 of 7/10/78):

(i) for a period of three years on new residential buildings;
A 40 percent tax rate is applied on profits. A minimum payment of 1 percent of turnover is levied on enterprises whose earnings are less than the turnover multiplied by a coefficient equal to 0.02222 .

After the expiration of the ten-year exemption period, a 15 percent rate is applied.

$$
\begin{array}{cc}
\text { Marginal Tax Rates } & \text { Rate } \\
\text { Income (FBu) } & \text { (percent) } \\
0-200,000 & 20 \\
200,001-400,000 & 25 \\
400,001-600,000 & 30 \\
600,001-800,000 & 40 \\
800,001-1,000,000 & 45 \\
1,000,001-1,500,000 & 50 \\
1,500,001 \text { and over } & 60
\end{array}
$$

An overall ceiling of 50 percent of taxable income applies. 


\section{Burundi: Summary of the Tax System, End-June 1998 (All amounts in Burundi francs)}

\begin{tabular}{|c|c|c|c|c|}
\hline Tax & Nature of Tax & Exemptions and Deductions & \multicolumn{2}{|l|}{ Rates } \\
\hline & & $\begin{array}{l}\text { (ii) for a period of four years on } \\
\text { new commercial, industrial, and } \\
\text { craft buildings; and } \\
\text { (iii) for a period of two years on } \\
\text { new buildings, other than those } \\
\text { mentioned above. } \\
\text { Deductions: } \\
\text { - A standard deduction of } 20 \text { per- } \\
\text { cent of gross rent. } \\
\text { - Mortgage interest paid to banks. }\end{array}$ & & \\
\hline $\begin{array}{l}\text { 1.3 Tax on capital income } \\
\text { (Tax Code II, Impôt } \\
\text { mobilier, Arts. 13-26). }\end{array}$ & $\begin{array}{l}\text { Annual schedular tax on dividends, } \\
\text { interest, and other revenue derived } \\
\text { from equity ownership in compa- } \\
\text { nies. Distributed income of foreign } \\
\text { companies is set on an inclusive } \\
\text { basis at } 48 \text { percent of all net profits. } \\
\text { The tax is withheld at source by the } \\
\text { companies. }\end{array}$ & $\begin{array}{l}\text { Exemptions: } \\
\text { - Those granted under the } \\
\text { Investment Code. } \\
\text { - Enterprises operating under the } \\
\text { free zone regime. }\end{array}$ & 20 percent. & \\
\hline $\begin{array}{l}1.4 \text { Tax on other income } \\
\text { (Tax Code II, } \\
\text { Impôt sur les revenus } \\
\text { professionnels, } \\
\text { Arts. 27-94). }\end{array}$ & $\begin{array}{l}\text { Annual schedular tax on all profits, } \\
\text { wage income, income from liberal } \\
\text { professions, or any other income, } \\
\text { the source of which is an activity on } \\
\text { Burundi territory and which is not } \\
\text { yet taxed under another schedular } \\
\text { tax. } \\
\text { Income in kind is included in the } \\
\text { tax base. } \\
\text { Free housing is valued at } 10 \text { percent } \\
\text { of the revenues obtained in } \\
\text { connection with the activity for } \\
\text { which these housing facilities are } \\
\text { granted. } \\
\text { The tax is withheld at source on } \\
\text { salaries above FBu } 100,500 \text { per } \\
\text { annum. }\end{array}$ & $\begin{array}{l}\text { Exemptions: } \\
\text { - Income covered under } \\
\text { intemational conventions ratified } \\
\text { by Burundi is exempt. } \\
\text { - Exemptions under the Investment } \\
\text { Code apply. } \\
\text { - Pensions, royalties, and the allow- } \\
\text { ances that were paid in } \\
\text { accordance with the legislation } \\
\text { regulating the payment of these } \\
\text { funds are exempt. } \\
\text { - Individual enterprises under the } \\
\text { free zone regime are exempt for } \\
\text { ten years. }\end{array}$ & $\begin{array}{r}\text { Marginal Tax Rates } \\
\text { Turnover (FBu) } \\
0-100,000 \\
100,001-200,000 \\
200,001-300,000 \\
300,001-400,000 \\
400,001-500,000 \\
500,001-600,000 \\
600,001-700,000 \\
700,001-800,000 \\
800,001-900,000 \\
900,001-1,000,000 \\
1,000,001-2,000,000 \\
2,000,001-3,000,000 \\
3,000,001-4,000,000 \\
4,000,001 \text { and over } \\
\text { A rate of } 1 \text { percent is ap } \\
\text { individual establishmen } \\
\text { realized earnings that ar } \\
\text { the turnover multiplied } \\
\text { coefficient equal to } 0.02\end{array}$ & 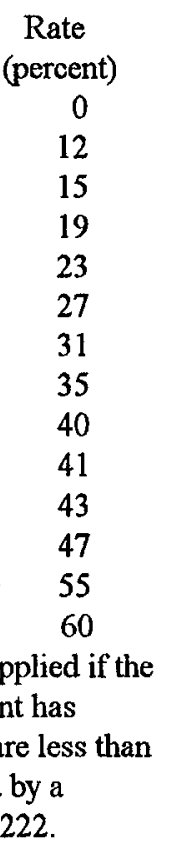 \\
\hline & & $\begin{array}{l}\text { Deductions: } \\
\text { - Expenses necessary to obtain the } \\
\text { taxable incomes and to maintain } \\
\text { their regularity are deductible. }\end{array}$ & $\begin{array}{l}\text { For coffee exports, the } n \\
\text { tax is equal to the turno } \\
\text { multiplied by } 0.25 \text { perc } \\
\text { of } 1 \text { percent. }\end{array}$ & $\begin{array}{l}\text { minimum } \\
\text { over figure } \\
\text { cent, instead }\end{array}$ \\
\hline
\end{tabular}




\section{Burundi: Summary of the Tax System, End-June 1998}

(All amounts in Burundi francs)

\begin{tabular}{|c|c|c|}
\hline $\operatorname{Tax}$ & Nature of Tax & Exemptions and Deductions \\
\hline & & $\begin{array}{l}\text { Expenses unrelated to the exer- } \\
\text { cise of the profession, such as } \\
\text { travel, medical, and canteen } \\
\text { expenses, and contributions to } \\
\text { social security are deductible; for } \\
\text { liberal professions, these expen- } \\
\text { ses are limited to a maximum of } \\
25 \text { percent or FBu 300,000, } \\
\text { whichever is higher. }\end{array}$ \\
\hline$\cdot$ & & $\begin{array}{l}\text { Contractual withholdings for } \\
\text { pension, life insurance, social } \\
\text { security, or profit funds are } \\
\text { considered as professional } \\
\text { expenses and are deductible. }\end{array}$ \\
\hline$\cdots$ & & $\begin{array}{l}\text { Losses incurred by liberal } \\
\text { professions during the past four } \\
\text { years are deductible. }\end{array}$ \\
\hline & & $\begin{array}{l}\text { Dependents' deduction is } \mathrm{FBu} \\
1,350 \text { per person, up to a } \\
\text { maximum of four persons. }\end{array}$ \\
\hline & & $\begin{array}{l}\text { Individual export enterprises can } \\
\text { deduct } 50 \text { percent of their export } \\
\text { profits. }\end{array}$ \\
\hline
\end{tabular}

\section{Taxes on property}

2.1 Real estate (Tax Code I, Impôt foncier, Arts. 2-38).
A specific tax payable by the owner Exemptions:

or the person having the use of a property. In November 1983, it was decided to transfer this tax to the local authorities. The tax has been transferred to the Municipality of Bujumbura (Law No. 1/002 of 2/4/84), and to all communes (Law No. $1 / 003$ of $1 / 8 / 87$ ).
- Those provided by the Investment Code.

- Agricultural land under cultivation or used as pasture for cattle.

- Buildings used by the government or by nonprofit organizations, whether owned by them or rented to them; sports fields; buildings used for religious purposes.
A tax of 0.55 percent of the factory price is levied on the earnings of wholesalers of beer and soft drinks.

For individual enterprises under the free zone regime, after the expiration of the ten-year exemption period, a 15 percent rate is applied.

Other Income (FBu) Rate (percent)

Occasional incomes

End-career allowances

$$
0-500,000 \quad 15
$$
$500,000+$

Income collected after end of professional activity
For built-on property, the following rates apply in Bujumbura and elsewhere:

- FBu $36 / \mathrm{m}^{2}$ for solid-construction buildings.

- FBu $24 / \mathrm{m}^{2}$ for semisolid buildings.

- $\mathrm{FBu} 15 / \mathrm{m}^{2}$ for provisional buildings. 


\section{Burundi: Summary of the Tax System, End-June 1998 (All amounts in Burundi francs)}

\begin{tabular}{|c|c|c|c|}
\hline \multicolumn{2}{|r|}{$\operatorname{Tax}$} & Nature of Tax & Exemptions and Deductions \\
\hline & & & $\begin{array}{l}\text { Properties belonging to } \\
\text { individuals with less than } \mathrm{FBu} \\
36,000 \text { annual income. } \\
\text { - In addition, buildings for four } \\
\text { years after their construction is } \\
\text { finished. }\end{array}$ \\
\hline 2.2 & $\begin{array}{l}\text { Cattle tax (Tax Code } \\
\text { IV, Impôt sur le } \\
\text { gros bétail, } \\
\text { Arts. 1-22). }\end{array}$ & $\begin{array}{l}\text { A tax owed by the cattle owners. At } \\
\text { the beginning of } 1979 \text {, both the } \\
\text { collection and revenue of this tax } \\
\text { were handed over to local } \\
\text { authorities. }\end{array}$ & None. \\
\hline
\end{tabular}

3. Taxes on domestic goods and services

3.1 Transactions tax Decree Law No.1/04 of 1/31/89 and Law No. 1/005 of 4/31/94).
Tax applicable only once on imports, manufacturing services, sales of buildings, and business premises. The tax paid earlier is deductible in the case of manufacturing, imports, restaurants, and construction work. However, taxes paid on fixed capital and business expenses are not deductible. Tax credit can be reimbursed.
Exemptions and deductions:

- Vehicles for commercial transportation.

- Inputs and equipment for agriculture and livestock; receipts of stadiums, transports, and international tariffs.

- Medical care, laboratory expenses, and medical biology.

- Inputs and equipments of enterprises operating under the free zone regime for exports.

3.2 Excise taxes on beer An ad valorem tax on the consump- Exemption: and soft drinks (OM 540/152 of March 6, 1992 in tion of local industrially made execution of Decree beverages. Duties on the consumpLaw No. 1/02 dated duty. February 8, 1992). tion of imported alcoholic beverages are incorporated in the fiscal import

- Products intended for export.

3.3 Excise tax on A tax on the consumption of locally None. tobacco. produced tobacco.

Primus Beer: 100 percent ad valorem tax on an ex factory price basis.

Amstel Beer: 30 percent ad valorem tax on an ex factory price basis.

Fanta, Coca Cola, Tonic, and other soft drinks: 30 percent ad valorem tax for a 24-bottle case on an ex factory price basis.

For other properties, the following rates apply in Bujumbura and where: equipment, $\mathrm{FBu} 2 / \mathrm{m}^{2}$.

For areas with medium equipment, $\mathrm{FBu} 3 / \mathrm{m}^{2}$.

For highly equipped areas, FBu 4/m².

FBu 200 per head.

7 percent on bank operations, sales of assets, sales of agricultural products, fishing, and livestock; 15 percent on other goods and services.

The transaction tax on beer, soft beverages, and tobacco is included in the ad valorem excise taxes specified below.

Ad valorem tax at 53 percent on a factory price basis. 


\section{Burundi: Summary of the Tax System, End-June 1998}

(All amounts in Burundi francs)

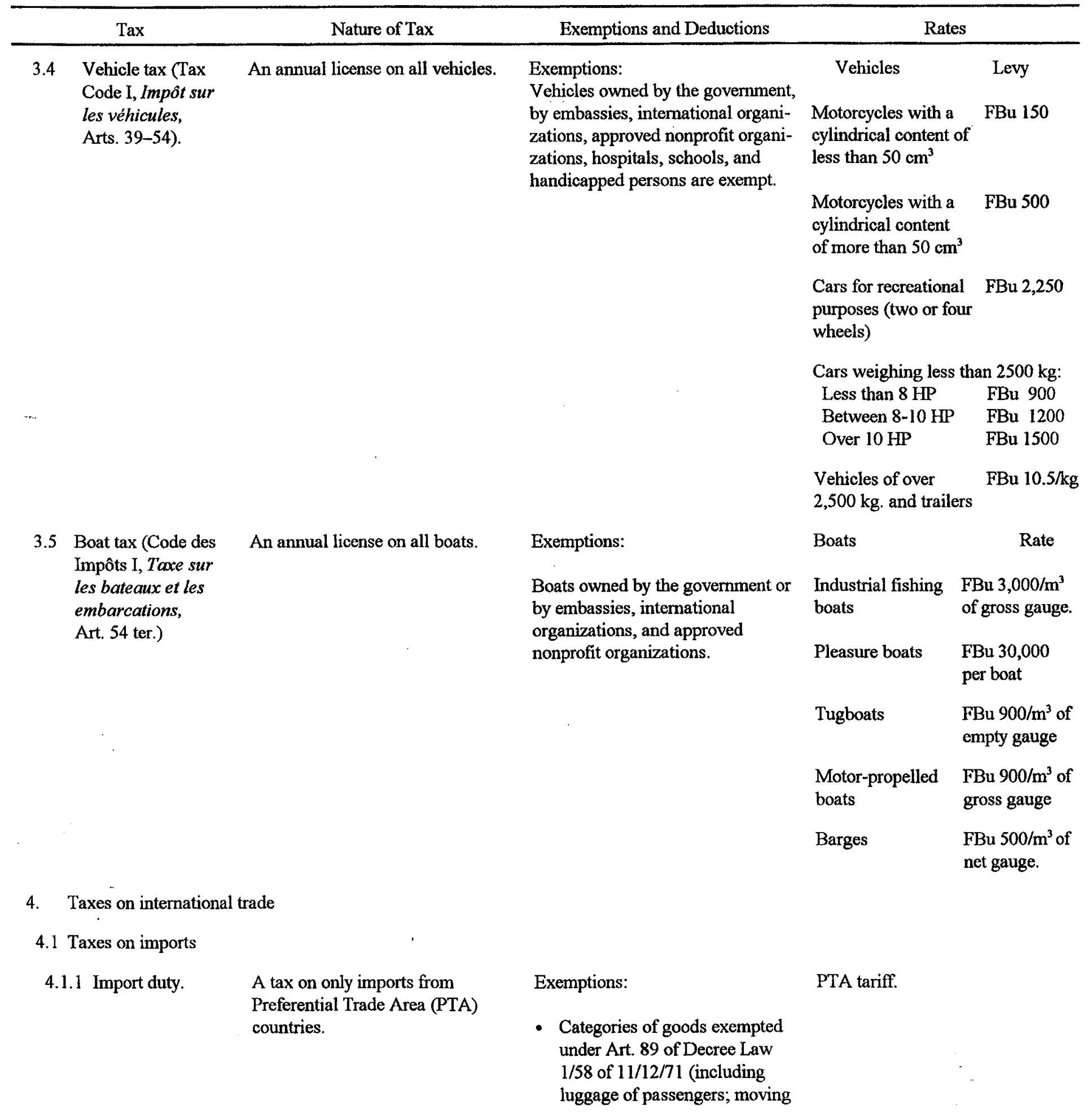




\section{Burundi: Summary of the Tax System, End-June 1998 \\ (All amounts in Burundi francs)}

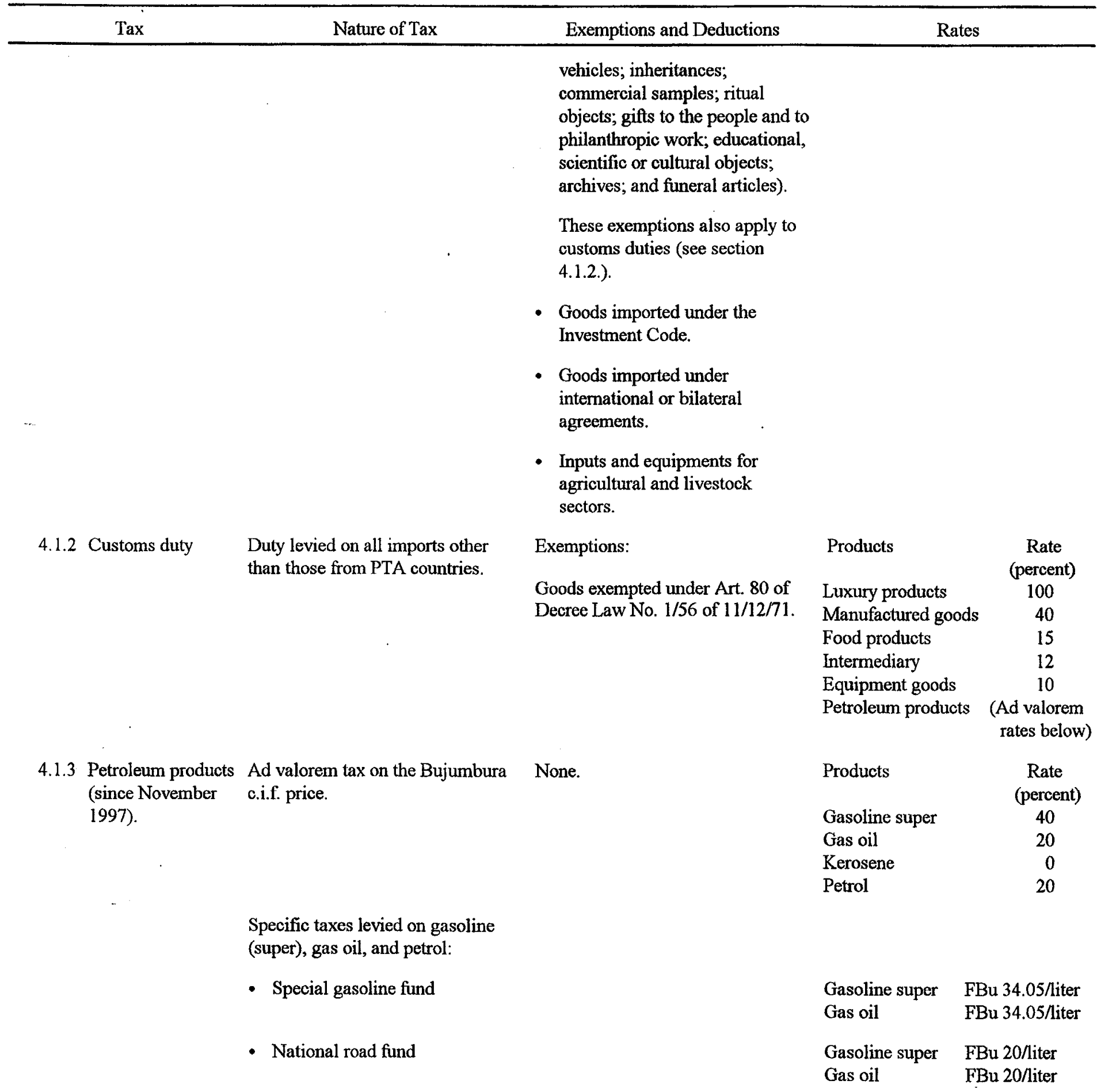




\section{Burundi: Summary of the Tax System, End-June 1998 (All amounts in Burundi francs)}

\begin{tabular}{|c|c|c|c|c|}
\hline & $\operatorname{Tax}$ & Nature of Tax & Exemptions and Deductions & Rates \\
\hline 4.1 .4 & Service tax. & $\begin{array}{l}\text { This tax, the proceeds of which are } \\
\text { assumed to compensate for the } \\
\text { administrative expenditures of } \\
\text { assessing and collecting the import } \\
\text { duties, is levied on all imports. The } \\
\text { tax was called statistical tax until } \\
\text { December } 1995 \text { when its name was } \\
\text { changed. }\end{array}$ & $\begin{array}{l}\text { Exemptions: } \\
\text { Imports under diplomatic franchise. }\end{array}$ & 6 percent of the c.i.f. value. \\
\hline \multicolumn{5}{|c|}{ 4.2 Taxes on exports } \\
\hline 4.2 .1 & Export duties. & $\begin{array}{l}\text { Taxes levied on a range of exports } \\
\text { (1,093 tariff positions against } 2,333 \\
\text { for the import tariff). The export } \\
\text { tariff has one column only. }\end{array}$ & $\begin{array}{l}\text { Reimbursement of taxes paid on } \\
\text { inputs: Decree Law No. } 1 / 012 \text { of } \\
4 / 15 / 88 \text { on export promotion. }\end{array}$ & $\begin{array}{l}\text { The generally applicable rate is } \\
5 \text { percent. Most export duties are } \\
\text { ad valorem. For green coffee, the } \\
\text { export tax is } 31 \text { percent. }\end{array}$ \\
\hline$\cdots$ & & & $\begin{array}{l}\text { Simplified drawback of } 10 \text { percent } \\
\text { on f.o.b. value of exported goods. }\end{array}$ & \\
\hline 5. & Other taxes & $\cdot$ & & \\
\hline 5.1 & $\begin{array}{l}\text { Stamp duties } \\
\text { (Droits d'en- } \\
\text { registrement, } \\
\text { Decree Law } \\
\text { No. } 1 / 43 \text { of } \\
6 / 11 / 70 \text { ). }\end{array}$ & $\begin{array}{l}\text { Fixed duties on certificates of } \\
\text { registration and on related } \\
\text { registrations. } \\
\text { - Proportional duties on the } \\
\text { establishment and transfer of } \\
\text { real property, as well as on } \\
\text { mortgages. }\end{array}$ & $\begin{array}{l}\text { Exemptions: } \\
\text { - Government and subordinate } \\
\text { agencies are exempt. } \\
\text { - Donations to religious and } \\
\text { scientific organizations and the } \\
\text { like are exempted from } \\
\text { proportional duties. }\end{array}$ & $\begin{array}{l}\text { The following rates are applied: } \\
\text { - FBu } 500 \text { for the first page. } \\
\text { - FBu } 125 \text { for each following } \\
\text { page. } \\
\text { - FBu } 250 \text { for the registration of a } \\
\text { right. } \\
100 \text { percent for transfers with } \\
\text { reduced rates for direct line } \\
\text { relations or those of spouse. } \\
\text { 2.5 percent for registration of a } \\
\text { mortgage. }\end{array}$ \\
\hline
\end{tabular}

